Article

\title{
Calculation of Five Thermodynamic Molecular Descriptors by Means of a General Computer Algorithm Based on the Group-Additivity Method: Standard Enthalpies of Vaporization, Sublimation and Solvation, and Entropy of Fusion of Ordinary Organic Molecules and Total Phase-Change Entropy of Liquid Crystals
}

\author{
Rudolf Naef ${ }^{1, *}$ and William E. Acree Jr. ${ }^{2}$ \\ 1 Department of Chemistry, University of Basel, Basel 4003, Switzerland \\ 2 Department of Chemistry, University of North Texas, Denton, TX 76203, USA; acree@unt.edu \\ * Correspondence: rudolf.naef@unibas.ch; Tel.: +41-619-119-273
}

Received: 22 May 2017; Accepted: 22 June 2017; Published: 25 June 2017

\begin{abstract}
The calculation of the standard enthalpies of vaporization, sublimation and solvation of organic molecules is presented using a common computer algorithm on the basis of a group-additivity method. The same algorithm is also shown to enable the calculation of their entropy of fusion as well as the total phase-change entropy of liquid crystals. The present method is based on the complete breakdown of the molecules into their constituting atoms and their immediate neighbourhood; the respective calculations of the contribution of the atomic groups by means of the Gauss-Seidel fitting method is based on experimental data collected from literature. The feasibility of the calculations for each of the mentioned descriptors was verified by means of a 10-fold cross-validation procedure proving the good to high quality of the predicted values for the three mentioned enthalpies and for the entropy of fusion, whereas the predictive quality for the total phase-change entropy of liquid crystals was poor. The goodness of fit $\left(Q^{2}\right)$ and the standard deviation $(\sigma)$ of the cross-validation calculations for the five descriptors was as follows: 0.9641 and $4.56 \mathrm{~kJ} / \mathrm{mol}$ ( $N=3386$ test molecules) for the enthalpy of vaporization, 0.8657 and $11.39 \mathrm{~kJ} / \mathrm{mol}(N=1791)$ for the enthalpy of sublimation, 0.9546 and $4.34 \mathrm{~kJ} / \mathrm{mol}(N=373)$ for the enthalpy of solvation, 0.8727 and $17.93 \mathrm{~J} / \mathrm{mol} / \mathrm{K}(N=2637)$ for the entropy of fusion and 0.5804 and $32.79 \mathrm{~J} / \mathrm{mol} / \mathrm{K}(N=2643)$ for the total phase-change entropy of liquid crystals. The large discrepancy between the results of the two closely related entropies is discussed in detail. Molecules for which both the standard enthalpies of vaporization and sublimation were calculable, enabled the estimation of their standard enthalpy of fusion by simple subtraction of the former from the latter enthalpy. For 990 of them the experimental enthalpy-of-fusion values are also known, allowing their comparison with predictions, yielding a correlation coefficient $R^{2}$ of 0.6066 .
\end{abstract}

Keywords: enthalpy of vaporization; enthalpy of sublimation; enthalpy of solvation; entropy of fusion; total phase-change entropy; tpc entropy; group-additivity method

\section{Introduction}

The reliable prediction of certain properties/descriptors of a molecule prior to its synthetic preparation has always been the goal of theoretical and experimental scientists, be it that they wanted to focus their experimental working hours on the synthesis of worthwhile compounds, be it that they 
wanted to verify their experimental results by means of the predictions. Among the many approaches, from the most elaborate ones such as the time-consuming ab initio methods to the fastest semiempirical self-consistent field procedures, one has turned out to be the most versatile and accurate and is not even quantum-theory-related: the atomic group-additivity method. A recent paper [1] demonstrated its versatility in that it enabled the calculation of mutually totally unrelated descriptors such as heat of combustion, solubility, refractivity, polarizability and toxicity by means of one single computer algorithm. This approach marks the endpoint, so to speak, of the various earlier group-additivity methods focusing on specific fields of application such as the prediction of the $\log \mathrm{P}_{\mathrm{O} / \mathrm{W}}$ values $[2,3]$, the molar refractivity [4], the molecular polarizability [5,6], or-closer to the present goal-the "simultaneous" evaluation of the logP, the aqueous solubility and the brain/blood distribution ratio $\log B B$ using individual parameter sets [7]. It is no secret, however, that the unsuccessful attempts in paper [1] to reliably predict just the latter descriptor, $\log B B$, put a damper on the expectation of a universal applicability of the present atomic group-additivity method. Yet, the exceptionally high prediction quality for the heat of combustion values across the entire structural spectrum of compounds presented in paper [1]—showing a cross-validated correlation coefficient of better than 0.9999 for 1965 compounds - at least gave rise to the hope that this method might successfully be extended to further thermodynamic descriptors.

The standard enthalpies of vaporization and sublimation were the first targets to be examined, not only because of their importance in chemical and environmental science, but also because a great deal of groundwork had already been done by Acree, Jr. and Chickos [8], who collected a large number of experimental vaporization and sublimation data covering more than a century. Several attempts to estimate the standard enthalpies of vaporization and sublimation have already been published: Roux et al. [9] evaluated the standard phase-change enthalpies of molecules from their experimental phase-change enthalpies at any given temperatures using their estimated heat capacity at room temperature. In cases where the number of experimental data was insufficient, they extrapolated the data from compounds with known experimental values. This estimation method, however, was limited to the vaporization enthalpy of liquid hydrocarbons. Similarly, Chickos et al. [10,11] estimated the vaporization enthalpies of larger even-numbered linear $n$-alkanes from a series of smaller ones [12,13] using their temperature dependence of the gas chromatographic retention time. A further indication of the potential applicability of the group-additivity method to predict the heats of vaporization and sublimation was found in the high correlation of the chain length of the homologues of saturated and unsaturated fatty acids with their experimental values [14].

Determination of the enthalpy of solvation has recently been based on the Abraham solute parameters model [15-18], the model consisting of a linear equation of five parameters relating to the molecule's excess molar refraction, the polarity/dipolarity, solute hydrogen-bond acidity and hydrogen-bond basicity, and the McGowan (i.e., molecular) volume. These parameters have been derived from the molecular structure of a series of compounds using multilinear regression analysis and artificial neural networks [19]. Earlier, Cabani et al. [20] described a group-contribution method for the estimation of the enthalpy, Gibbs free energy and heat capacity of liquids of non-ionic solutes in water, limiting the method for the calculation of the group contributions to compounds with not more than one heteroatom and then applying correction parameters for molecules containing more than one heteroatom.

The entropy of fusion (often-and more logically-called entropy of phase change or even better: entropy of melting) of ordinary organic molecules as well as its special manifestation with liquid crystals, called total phase-change entropy, generally mean the entropy of the transition of a molecule from its most stable crystalline form to the isotropic melt. While for ordinary molecules this transition in most cases occurs in one step or two consecutive steps upon addition of thermal energy, this process is much more complex with liquid crystals in that they know several intermediate, semi-crystalline phases melting at considerably different temperatures. In the first case, occurrence of more than one melting step may be explained by polymorphism of the crystalline form, their various 
polymorphic forms often showing distinct differences in their fusion enthalpies. In the second, the various semi-crystalline forms can be stable over a considerable temperature range, thus consuming a large amount of thermal energy prior to their next phase change. The thermodynamic consequences of the difference in the melting processes between ordinary molecules and compounds exhibiting liquid crystal properties forced Chickos et al. [21] and Acree, Jr. et al. [22] to treat these two categories of compounds as separate entities in their collective volumes.

The present work, being a continuation of the principle to calculate the molecular descriptors published earlier [1], will show the extendability of the approach to reliably predict the enthalpies of vaporization, sublimation and solvation, as well as the entropy of fusion. In order to clearly distinguish the phase-change entropy of ordinary compounds from that of liquid crystals, the term "entropy of fusion" will remain reserved for the former, while for the latter the well-established term "total phase-change entropy" will be used throughout.

\section{General Procedure}

All the calculations are based on a knowledge database encompassing at present more than 28,500 records, containing the compounds in their geometry-optimized 3D form and carrying all the required (and several more) data. The database includes—besides ordinary organic molecules-organic salts, ionic liquids, liquid crystals and metal-organic compounds.

The algorithm for the calculation of the present descriptors follows the atom-group additivity principle outlined in detail in the earlier paper [1]. Consequently, the naming and meaning of the atom groups in the parameters tables is the same, the tables being complemented by further atom groups, where necessary, following the rules described in Table 1 of [1]. The results of the evaluation of the atom-group contributions are stored in a separate parameters list for each descriptor. The only difference to the earlier work lies in the addition of a further special group as a consequence of attempts to optimize calculations of the group contributions for the entropies of fusion, where it turned out that the difference between the experimental values of open-chained and cyclical compounds was not resolvable by the given ordinary atom groups themselves. Therefore, a special group called "Endocyclic bonds" has been introduced which counts the number of endocyclic bonds in a molecule but is restricted to single bonds to take account of their reduced freedom of mobility within a ring system (bonds of higher order are by themselves restricted). Its treatment within the calculation is identical to the one described for all the other special groups.

Once the group contributions have been evaluated as described earlier, the prediction of the descriptors follows the general Equation (1), where $a_{i}$ and $b_{j}$ are the contributions, $A_{i}$ is the number of occurrences of the $i$ th atom group, $B_{j}$ is the number of occurrences of the special groups and $C$ is a constant:

$$
Y=\sum_{i} a_{i} A_{i}+\sum_{j} b_{j} B_{j}+C
$$

It is immediately evident that this equation excludes prediction of descriptors for molecules for which not all atom groups are present in the corresponding parameters table. Yet, a further limitation is given by the condition that only atom groups are valid for consideration that have been represented by at least three independent molecules in the parameters-evaluation process. The number of molecules representing a given atom group is listed in the rightmost column of the parameters tables shown below. The remaining atom groups represented by less than three molecules are kept in the parameters tables solely for future use in this continuing project (and to invite researchers experimenting in these areas to focus on compounds carrying these atom groups). The calculations are generally restricted to molecules containing the elements $\mathrm{H}, \mathrm{B}, \mathrm{C}, \mathrm{N}, \mathrm{O}, \mathrm{P}, \mathrm{S}, \mathrm{Si}$ and/or halogen.

Plausibility tests have been carried out for each of the atom-group additivity parameters evaluations applying a 10-fold cross-validation procedure as described in [1], making sure that each compound has been used once as a test sample in the process. The results of these calculations are condensed in row $\mathrm{A}$ to $\mathrm{H}$ at the end of each parameters table. In the corresponding correlation 
diagrams (Figure 7) and histograms presented below the results of the cross-validation calculations are superpositioned in red over the training data.

\section{Results}

\subsection{General Remarks}

(1) The experimental values of enthalpies and entropies are temperature-dependent. Any relationship within these properties or with other ones only make sense if they are referenced to the same temperature. The usual temperature of reference is $298.15 \mathrm{~K}$, and thus it was ensured in this work that experimental data from literature were only accepted if they had been either measured at or adjusted to the standard temperatur of $298.15 \mathrm{~K}$ and standard pressure of $100 \mathrm{kPa}$.

(2) All lists of molecules used in the atom-group parameters evaluations have been collected in standard SDF files, stored in the supplementary material, ready to be imported by external chemistry software. The supplementary material also provides the lists of results containing molecule names, experimental, training and cross-validation values. Beyond this, it also contains lists of experimental outliers.

\subsection{Enthalpy of Vaporization}

Experimental data of vaporization enthalpies have essentially been extracted for this work from the large collection of Acree, Jr. and Chickos [8] and Chickos et al. [10-14], supplemented by recent data from a number of further authors publishing experimental vaporization values of several acetophenones [23], aliphatic tertiary amines [24], azidomethyl- $N$-nitrooxazolidines [25], benzamides [26], benzocaine [27], bisabolol and menthol [28], crown ethers [29], $N, N$-dialkyl monoamides [30], fenpropidin and phencyclidine [31], flavors [32], long-chain fluorinated alcohols [33], whiskey- and metha-lactone [34], halogenated fluorenes [35], ibuprofen and naproxen [36], imidazo[1,2-a]pyrazine and phthalazine [37], insect pheromones [38], morpholines [39], organo(thio)phosphates [40], dialkyl phthalates [41], nitrogen heteroaromatics [42], phenylimidazoles [43], 2-acetylthiophene [44], dicarboxylic n-pentyl esters [45], and cyclic amines, ethers and alcohols [46]. The result of the atom-group parameters, based on 3581 compounds, is summarized in Table 1. Several tentative calculations with or without inclusion of certain special groups outlined in Table 2 of the earlier paper [1] revealed a minor improvement of the goodness of fit upon inclusion of the "atom group" responsible for intramolecular acid-base bonds, named "H/H Acceptor", as well as of those reserved for saturated and unsaturated pure hydrocarbons, called "Alkane/No. of C atoms" and "Unsaturated HC/No. of C atoms", which add a correction value for each carbon atom.

Table 1. Atom Groups and their Contributions (in $\mathrm{kJ} / \mathrm{mol}$ ) for Heat-of-Vaporization Calculations.

\begin{tabular}{cccccc}
\hline Entry & Atom Type & Neighbours & Contribution & Occurrences & Molecules \\
\hline 1 & Const & & 8.61 & 3581 & 3581 \\
2 & $\mathrm{~B}$ & $\mathrm{C} 3$ & 21.55 & 2 & 2 \\
3 & $\mathrm{~N}$ & $\mathrm{~N} C l$ & 33.19 & 1 & 1 \\
4 & $\mathrm{NCl} 2$ & 28.59 & 1 & 1 \\
5 & $\mathrm{~B}$ & $\mathrm{O} 2 \mathrm{Cl}$ & 28.23 & 2 & 2 \\
6 & $\mathrm{~B}$ & $\mathrm{OCl} 2$ & 76.73 & 1 & 1 \\
7 & $\mathrm{~B}$ & $\mathrm{H} 3 \mathrm{C}$ & 3.07 & 4 & 4 \\
8 & $\mathrm{~B}$ & $\mathrm{H} 3 \mathrm{~N}$ & 15.65 & 5380 & 2388 \\
9 & $\mathrm{C} \mathrm{sp}$ & $\mathrm{H} 3 \mathrm{~N}(+)$ & 31.33 & 2 & 2 \\
10 & $\mathrm{C} \mathrm{sp}$ & $\mathrm{H} 3 \mathrm{O}$ & 16.71 & 372 & 263 \\
11 & $\mathrm{C} \mathrm{sp}$ & $\mathrm{H} 3 \mathrm{~S}$ & 14.44 & 31 & 25 \\
12 & $\mathrm{C} \mathrm{sp}$ & $\mathrm{H} 3 \mathrm{P}$ & 9.04 & 6 & 4 \\
13 & $\mathrm{C} \mathrm{sp}$ & $\mathrm{C} \mathrm{sp}$ & & & 2 \\
\hline
\end{tabular}


Table 1. Cont

\begin{tabular}{|c|c|c|c|c|c|}
\hline Entry & Atom Type & Neighbours & Contribution & Occurrences & Molecules \\
\hline 14 & $\mathrm{Csp} \mathrm{sp}^{3}$ & H3Si & 5.87 & 136 & 53 \\
\hline 15 & $\mathrm{C} \mathrm{sp}^{3}$ & $\mathrm{H} 2 \mathrm{BC}$ & -3.07 & 6 & 2 \\
\hline 16 & $\mathrm{C} \mathrm{sp}^{3}$ & $\mathrm{H} 2 \mathrm{C} 2$ & 4.67 & 10,588 & 2030 \\
\hline 17 & $\mathrm{C} \mathrm{sp}^{3}$ & $\mathrm{H} 2 \mathrm{CN}$ & 15.00 & 430 & 243 \\
\hline 18 & $\mathrm{Csp}{ }^{3}$ & $\mathrm{H} 2 \mathrm{CN}(+)$ & 29.15 & 10 & 9 \\
\hline 19 & $\mathrm{C} \mathrm{sp}^{3}$ & $\mathrm{H} 2 \mathrm{CO}$ & 15.79 & 1147 & 779 \\
\hline 20 & $\mathrm{Csp}$ & $\mathrm{H} 2 \mathrm{CS}$ & 15.50 & 159 & 101 \\
\hline 21 & $\mathrm{Csp}$ & $\mathrm{H} 2 \mathrm{CP}$ & 6.67 & 6 & 2 \\
\hline 22 & $\mathrm{Csp}^{3}$ & $\mathrm{H} 2 \mathrm{CF}$ & 6.20 & 11 & 11 \\
\hline 23 & $\mathrm{Csp}$ & $\mathrm{H} 2 \mathrm{CCl}$ & 14.13 & 76 & 65 \\
\hline 24 & $\mathrm{Csp}$ & $\mathrm{H} 2 \mathrm{CBr}$ & 16.69 & 24 & 21 \\
\hline 25 & $\mathrm{Csp} \mathrm{sp}^{3}$ & $\mathrm{H} 2 \mathrm{CJ}$ & 20.90 & 29 & 26 \\
\hline 26 & $\mathrm{Csp}{ }^{3}$ & $\mathrm{H} 2 \mathrm{CSi}$ & 2.01 & 134 & 54 \\
\hline 27 & $\mathrm{Csp}$ & $\mathrm{H} 2 \mathrm{~N} 2$ & 28.27 & 5 & 3 \\
\hline 28 & $\mathrm{Csp}$ & $\mathrm{H} 2 \mathrm{NO}$ & 20.46 & 4 & 4 \\
\hline 29 & $\mathrm{Csp}$ & $\mathrm{H} 2 \mathrm{O} 2$ & 27.43 & 19 & 16 \\
\hline 30 & $\mathrm{Csp}{ }^{3}$ & $\mathrm{H} 2 \mathrm{OS}$ & 22.40 & 1 & 1 \\
\hline 31 & $\mathrm{Csp}$ & $\mathrm{H} 2 \mathrm{OF}$ & 18.90 & 1 & 1 \\
\hline 32 & $\mathrm{C} \mathrm{sp}^{3}$ & $\mathrm{H} 2 \mathrm{OCl}$ & 23.06 & 2 & 2 \\
\hline 33 & $\mathrm{Csp}$ & $\mathrm{H} 2 \mathrm{OSi}$ & 10.30 & 1 & 1 \\
\hline 34 & $\mathrm{Csp}{ }^{3}$ & $\mathrm{H} 2 \mathrm{~S} 2$ & 24.08 & 2 & 2 \\
\hline 35 & $\mathrm{Csp}{ }^{3}$ & $\mathrm{H} 2 \mathrm{SSi}$ & 6.66 & 9 & 9 \\
\hline 36 & $\mathrm{Csp}$ & $\mathrm{H} 2 \mathrm{Si} 2$ & 2.87 & 2 & 1 \\
\hline 37 & $C \mathrm{sp}^{3}$ & HC3 & 3.54 & 939 & 615 \\
\hline 38 & $\mathrm{Csp}$ & $\mathrm{HC} 2 \mathrm{~N}$ & 12.69 & 75 & 64 \\
\hline 39 & $\mathrm{Csp}$ & $\mathrm{HC} 2 \mathrm{~N}(+)$ & 28.39 & 3 & 3 \\
\hline 40 & $\mathrm{Csp}$ & $\mathrm{HC} 2 \mathrm{O}$ & 14.99 & 243 & 203 \\
\hline 41 & $\mathrm{Csp}{ }^{3}$ & $\mathrm{HC} 2 \mathrm{~S}$ & 13.61 & 26 & 22 \\
\hline 42 & $C \mathrm{sp}^{3}$ & $\mathrm{HC} 2 \mathrm{Si}$ & 7.20 & 6 & 4 \\
\hline 43 & $C \mathrm{sp}^{3}$ & $\mathrm{HC} 2 \mathrm{~F}$ & 5.96 & 7 & 6 \\
\hline 44 & $\mathrm{Csp}$ & $\mathrm{HC} 2 \mathrm{Cl}$ & 9.66 & 40 & 38 \\
\hline 45 & $\mathrm{Csp} \mathrm{sp}^{3}$ & $\mathrm{HC} 2 \mathrm{Br}$ & 12.12 & 21 & 16 \\
\hline 46 & $C \mathrm{sp}^{3}$ & HC2J & 18.79 & 4 & 4 \\
\hline 47 & $\mathrm{Csp}{ }^{3}$ & HCN2(+) & 47.10 & 3 & 3 \\
\hline 48 & $\mathrm{Csp}$ & $\mathrm{HCO} 2$ & 25.39 & 25 & 22 \\
\hline 49 & $\mathrm{Csp}{ }^{3}$ & $\mathrm{HCOCl}$ & 20.93 & 1 & 1 \\
\hline 50 & $\mathrm{Csp}$ & HCF2 & 7.10 & 15 & 14 \\
\hline 51 & $C \mathrm{sp}^{3}$ & $\mathrm{HCFCl}$ & 12.61 & 15 & 15 \\
\hline 52 & $\mathrm{C} \mathrm{sp}^{3}$ & $\mathrm{HCCl} 2$ & 16.96 & 23 & 22 \\
\hline 53 & $C \mathrm{sp}^{3}$ & $\mathrm{HCClBr}$ & 18.23 & 1 & 1 \\
\hline 54 & $\mathrm{Csp}$ & HNO2 & 32.31 & 1 & 1 \\
\hline 55 & $C \mathrm{sp}^{3}$ & $\mathrm{HO} 3$ & 37.33 & 4 & 4 \\
\hline 56 & $\mathrm{Csp} \mathrm{sp}^{3}$ & HOF2 & 17.06 & 7 & 7 \\
\hline 57 & $\mathrm{Csp}^{3}$ & $\mathrm{HOFCl}$ & 20.49 & 1 & 1 \\
\hline 58 & $\mathrm{Csp} \mathrm{sp}^{3}$ & $\mathrm{HSiCl} 2$ & 23.89 & 1 & 1 \\
\hline 59 & $C \mathrm{sp}^{3}$ & $\mathrm{C} 4$ & 1.92 & 335 & 274 \\
\hline 60 & $\mathrm{Csp}^{3}$ & $\mathrm{C} 3 \mathrm{~N}$ & 12.60 & 28 & 23 \\
\hline 61 & $\mathrm{Csp}$ & $\mathrm{C} 3 \mathrm{~N}(+)$ & 26.15 & 4 & 4 \\
\hline 62 & $\mathrm{Csp}$ & $\mathrm{C} 3 \mathrm{O}$ & 12.21 & 135 & 116 \\
\hline 63 & $C \mathrm{sp}^{3}$ & C3S & 13.69 & 18 & 16 \\
\hline 64 & $\mathrm{Csp} \mathrm{sp}^{3}$ & $\mathrm{C} 3 \mathrm{~F}$ & 2.94 & 31 & 19 \\
\hline 65 & $\mathrm{Csp} \mathrm{sp}^{3}$ & $\mathrm{C} 3 \mathrm{Cl}$ & 7.77 & 8 & 6 \\
\hline 66 & $\mathrm{Csp}^{3}$ & $\mathrm{C} 3 \mathrm{Br}$ & 11.95 & 3 & 3 \\
\hline 67 & $\mathrm{Csp}^{3}$ & C3J & 19.63 & 2 & 2 \\
\hline 68 & $\mathrm{Csp}{ }^{3}$ & $\mathrm{C} 2 \mathrm{NO}$ & 20.34 & 1 & 1 \\
\hline
\end{tabular}


Table 1. Cont.

\begin{tabular}{|c|c|c|c|c|c|}
\hline Entry & Atom Type & Neighbours & Contribution & Occurrences & Molecules \\
\hline 69 & $\mathrm{Csp} \mathrm{sp}^{3}$ & $\mathrm{C} 2 \mathrm{NF}$ & 8.88 & 1 & 1 \\
\hline 70 & $\mathrm{C} \mathrm{sp}^{3}$ & $\mathrm{C} 2 \mathrm{O} 2$ & 23.16 & 35 & 27 \\
\hline 71 & $\mathrm{C} \mathrm{sp}^{3}$ & $\mathrm{C} 2 \mathrm{OF}$ & 18.38 & 3 & 3 \\
\hline 72 & $\mathrm{C} \mathrm{sp}^{3}$ & $\mathrm{C} 2 \mathrm{~F} 2$ & 4.75 & 328 & 70 \\
\hline 73 & $\mathrm{Csp}{ }^{3}$ & $\mathrm{C} 2 \mathrm{FCl}$ & 8.73 & 5 & 5 \\
\hline 74 & $\mathrm{C} \mathrm{sp}^{3}$ & $\mathrm{C} 2 \mathrm{Cl} 2$ & 13.35 & 5 & 5 \\
\hline 75 & $\mathrm{C} \mathrm{sp}^{3}$ & CN3(+) & 46.89 & 3 & 3 \\
\hline 76 & $\mathrm{Csp} \mathrm{sp}^{3}$ & CNF2 & 15.25 & 15 & 6 \\
\hline 77 & $C \mathrm{sp}^{3}$ & CNF2(+) & 30.77 & 3 & 2 \\
\hline 78 & $\mathrm{Csp}$ & $\mathrm{CN} 2 \mathrm{~F}(+)$ & 28.25 & 4 & 3 \\
\hline 79 & $\mathrm{Csp}$ & $\mathrm{CO} 3$ & 28.48 & 6 & 6 \\
\hline 80 & $\mathrm{Csp} \mathrm{sp}^{3}$ & $\mathrm{COF} 2$ & 13.65 & 36 & 30 \\
\hline 81 & $\mathrm{Csp}{ }^{3}$ & $\mathrm{COCl} 2$ & 20.61 & 4 & 4 \\
\hline 82 & $\mathrm{Csp}$ & CSF2 & 12.70 & 2 & 1 \\
\hline 83 & $\mathrm{Csp}^{3}$ & CF3 & 2.96 & 147 & 90 \\
\hline 84 & $\mathrm{Csp}$ & $\mathrm{CF} 2 \mathrm{Cl}$ & 6.64 & 10 & 9 \\
\hline 85 & $C \mathrm{sp}^{3}$ & $\mathrm{CF} 2 \mathrm{Br}$ & 9.02 & 5 & 4 \\
\hline 86 & $\mathrm{Csp}$ & $\mathrm{CFCl} 2$ & 13.41 & 7 & 7 \\
\hline 87 & $\mathrm{Csp}$ & $\mathrm{CFClBr}$ & 17.37 & 1 & 1 \\
\hline 88 & $\mathrm{Csp}$ & $\mathrm{CCl} 3$ & 17.43 & 22 & 21 \\
\hline 89 & $\mathrm{Csp}{ }^{3}$ & NF3 & 14.48 & 5 & 4 \\
\hline 90 & $\mathrm{Csp}{ }^{3}$ & NF3(+) & -1.76 & 2 & 1 \\
\hline 91 & $\mathrm{Csp}$ & $\mathrm{N} 3 \mathrm{~F}(+)$ & 32.36 & 1 & 1 \\
\hline 92 & $C \mathrm{sp}^{3}$ & $\mathrm{O} 4$ & 38.15 & 2 & 2 \\
\hline 93 & $C \mathrm{sp}^{3}$ & $\mathrm{O} 2 \mathrm{~F} 2$ & 24.80 & 14 & 2 \\
\hline 94 & $\mathrm{Csp}$ & OF3 & 9.71 & 9 & 7 \\
\hline 95 & $\mathrm{Csp}$ & $\mathrm{OF} 2 \mathrm{Cl}$ & 17.84 & 2 & 2 \\
\hline 96 & $\mathrm{Csp}{ }^{3}$ & $\mathrm{OCl} 3$ & 27.40 & 2 & 2 \\
\hline 97 & $\mathrm{Csp}$ & PF3 & 2.73 & 2 & 1 \\
\hline 98 & $C \mathrm{sp}^{2}$ & $\mathrm{H} 2=\mathrm{C}$ & 2.17 & 182 & 170 \\
\hline 99 & $\mathrm{Csp} \mathrm{sp}^{2}$ & $\mathrm{HC}=\mathrm{C}$ & 5.03 & 1314 & 694 \\
\hline 100 & $C \mathrm{sp}^{2}$ & $\mathrm{HC}=\mathrm{N}$ & 8.81 & 15 & 15 \\
\hline 101 & $C \mathrm{sp}^{2}$ & $\mathrm{HC}=\mathrm{O}$ & 11.44 & 122 & 122 \\
\hline 102 & $C \mathrm{sp}^{2}$ & $\mathrm{H}=\mathrm{CN}$ & 17.18 & 103 & 57 \\
\hline 103 & $C \mathrm{sp}^{2}$ & $\mathrm{H}=\mathrm{CO}$ & 10.25 & 35 & 32 \\
\hline 104 & $C \mathrm{sp}^{2}$ & $\mathrm{H}=\mathrm{CS}$ & 8.20 & 49 & 35 \\
\hline 105 & $C \mathrm{sp}^{2}$ & $\mathrm{H}=\mathrm{CSi}$ & 10.77 & 4 & 4 \\
\hline 106 & $C \mathrm{sp}^{2}$ & $\mathrm{H}=\mathrm{CF}$ & -0.09 & 1 & 1 \\
\hline 107 & $C \mathrm{sp}^{2}$ & $\mathrm{H}=\mathrm{CCl}$ & 10.38 & 8 & 6 \\
\hline 108 & $\mathrm{Csp}{ }^{2}$ & $\mathrm{H}=\mathrm{CBr}$ & 13.73 & 1 & 1 \\
\hline 109 & $\mathrm{C} \mathrm{sp}^{2}$ & $\mathrm{HN}=\mathrm{N}$ & 30.13 & 39 & 39 \\
\hline 110 & $C \mathrm{sp}^{2}$ & $\mathrm{HN}=\mathrm{O}$ & 34.46 & 6 & 6 \\
\hline 111 & $\mathrm{Csp}{ }^{2}$ & $\mathrm{H}=\mathrm{NO}$ & 14.07 & 1 & 1 \\
\hline 112 & $C \mathrm{sp}^{2}$ & $\mathrm{H}=\mathrm{NS}$ & 18.07 & 2 & 2 \\
\hline 113 & $\mathrm{Csp} \mathrm{sp}^{2}$ & $\mathrm{HO}=\mathrm{O}$ & 18.86 & 14 & 12 \\
\hline 114 & $C \mathrm{sp}^{2}$ & $\mathrm{C} 2=\mathrm{C}$ & 5.27 & 220 & 190 \\
\hline 115 & $C \mathrm{sp}^{2}$ & $\mathrm{C} 2=\mathrm{N}$ & 8.22 & 15 & 14 \\
\hline 116 & $C \mathrm{sp}^{2}$ & $\mathrm{C} 2=\mathrm{O}$ & 13.59 & 149 & 140 \\
\hline 117 & $\mathrm{Csp}{ }^{2}$ & $\mathrm{C}=\mathrm{CN}$ & 15.36 & 14 & 10 \\
\hline 118 & $C \mathrm{sp}^{2}$ & $\mathrm{C}=\mathrm{CO}$ & 12.54 & 39 & 31 \\
\hline 119 & $C \mathrm{sp}^{2}$ & $\mathrm{C} 2=\mathrm{S}$ & 71.29 & 2 & 2 \\
\hline 120 & $\mathrm{C} \mathrm{sp}^{2}$ & $\mathrm{C}=\mathrm{CS}$ & 9.45 & 29 & 24 \\
\hline 121 & $\mathrm{Csp}^{2}$ & $\mathrm{C}=\mathrm{CF}$ & 2.72 & 11 & 5 \\
\hline 122 & $C \mathrm{sp}^{2}$ & $\mathrm{C}=\mathrm{CCl}$ & 5.83 & 8 & 5 \\
\hline 123 & $\mathrm{C} \mathrm{sp}^{2}$ & $\mathrm{C}=\mathrm{CBr}$ & 15.79 & 1 & 1 \\
\hline
\end{tabular}


Table 1. Cont.

\begin{tabular}{|c|c|c|c|c|c|}
\hline Entry & Atom Type & Neighbours & Contribution & Occurrences & Molecules \\
\hline 124 & $\mathrm{Csp} \mathrm{sp}^{2}$ & $=\mathrm{CN} 2$ & 9.12 & 3 & 2 \\
\hline 125 & $\mathrm{Csp} \mathrm{sp}^{2}$ & $\mathrm{CN}=\mathrm{N}$ & 28.80 & 16 & 16 \\
\hline 126 & $\mathrm{Csp}^{2}$ & $\mathrm{CN}=\mathrm{N}(+)$ & 11.32 & 2 & 2 \\
\hline 127 & $C \mathrm{sp}^{2}$ & $\mathrm{CN}=\mathrm{O}$ & 35.35 & 47 & 47 \\
\hline 128 & $C \mathrm{sp}^{2}$ & $\mathrm{C}=\mathrm{NO}$ & 22.79 & 5 & 5 \\
\hline 129 & $C \mathrm{sp}^{2}$ & $\mathrm{CN}=\mathrm{S}$ & 18.27 & 3 & 2 \\
\hline 130 & $\mathrm{C} \mathrm{sp}^{2}$ & $\mathrm{C}=\mathrm{NS}$ & 17.49 & 1 & 1 \\
\hline 131 & $\mathrm{Csp} \mathrm{sp}^{2}$ & $\mathrm{C}=\mathrm{NCl}$ & 11.93 & 1 & 1 \\
\hline 132 & $\mathrm{Csp} \mathrm{sp}^{2}$ & $=\mathrm{CNCl}$ & 22.67 & 2 & 1 \\
\hline 133 & $\mathrm{Csp} \mathrm{sp}^{2}$ & $\mathrm{CO}=\mathrm{O}$ & 17.20 & 684 & 594 \\
\hline 134 & $\mathrm{Csp}$ & $=\mathrm{COS}$ & 17.48 & 1 & 1 \\
\hline 135 & $\mathrm{Csp} \mathrm{sp}^{2}$ & $\mathrm{C}=\mathrm{OS}$ & 12.33 & 9 & 9 \\
\hline 136 & $\mathrm{Csp}$ & $=\mathrm{COF}$ & 15.53 & 1 & 1 \\
\hline 137 & $\mathrm{Csp} \mathrm{sp}^{2}$ & $\mathrm{C}=\mathrm{OCl}$ & 15.41 & 11 & 9 \\
\hline 138 & $\mathrm{Csp} \mathrm{sp}^{2}$ & $\mathrm{C}=\mathrm{OBr}$ & 22.28 & 3 & 3 \\
\hline 139 & $\mathrm{Csp} \mathrm{sp}^{2}$ & $\mathrm{C}=\mathrm{OJ}$ & 25.82 & 2 & 2 \\
\hline 140 & $\mathrm{Csp}$ & $=\mathrm{CF} 2$ & -0.26 & 3 & 3 \\
\hline 141 & $\mathrm{Csp}^{2}$ & $=\mathrm{CFCl}$ & 9.81 & 3 & 2 \\
\hline 142 & $\mathrm{Csp} \mathrm{sp}^{2}$ & $=\mathrm{CCl} 2$ & 17.52 & 6 & 5 \\
\hline 143 & $\mathrm{Csp}$ & $\mathrm{N} 2=\mathrm{N}$ & 29.25 & 2 & 2 \\
\hline 144 & $\mathrm{Csp} \mathrm{sp}^{2}$ & $\mathrm{~N} 2=\mathrm{O}$ & 35.05 & 3 & 3 \\
\hline 145 & $C \mathrm{sp}^{2}$ & $\mathrm{~N}=\mathrm{NS}$ & 13.50 & 5 & 5 \\
\hline 146 & $\mathrm{Csp}$ & $\mathrm{NO}=\mathrm{O}$ & 33.48 & 3 & 3 \\
\hline 147 & $\mathrm{Csp} \mathrm{sp}^{2}$ & $=\mathrm{NOCl}$ & 24.27 & 1 & 1 \\
\hline 148 & $\mathrm{Csp}$ & $\mathrm{NS}=\mathrm{S}$ & 44.39 & 2 & 2 \\
\hline 149 & $C \mathrm{sp}^{2}$ & $\mathrm{O} 2=\mathrm{O}$ & 31.57 & 13 & 13 \\
\hline 150 & $\mathrm{C} \mathrm{sp}^{2}$ & $\mathrm{O}=\mathrm{OCl}$ & 22.73 & 2 & 2 \\
\hline 151 & $C \mathrm{sp}^{2}$ & $\mathrm{~S} 2=\mathrm{S}$ & 34.03 & 1 & 1 \\
\hline 152 & $\mathrm{C}$ aromatic & $\mathrm{H}: \mathrm{C} 2$ & 4.64 & 4749 & 928 \\
\hline 153 & $\mathrm{C}$ aromatic & $\mathrm{H}: \mathrm{C}: \mathrm{N}$ & 11.74 & 118 & 70 \\
\hline 154 & $\mathrm{C}$ aromatic & $\mathrm{H}: \mathrm{C}: \mathrm{N}(+)$ & 22.04 & 2 & 1 \\
\hline 155 & C aromatic & H:N2 & 15.36 & 7 & 5 \\
\hline 156 & $\mathrm{C}$ aromatic & :C3 & 6.67 & 233 & 69 \\
\hline 157 & C aromatic & $\mathrm{C}: \mathrm{C} 2$ & 5.29 & 1053 & 618 \\
\hline 158 & $\mathrm{C}$ aromatic & $C: C: N$ & 9.94 & 38 & 30 \\
\hline 159 & C aromatic & $: \mathrm{C} 2 \mathrm{~N}$ & 14.44 & 140 & 115 \\
\hline 160 & C aromatic & $: \mathrm{C} 2 \mathrm{~N}(+)$ & 24.38 & 33 & 31 \\
\hline 161 & $\mathrm{C}$ aromatic & $: \mathrm{C} 2: \mathrm{N}$ & 10.60 & 21 & 14 \\
\hline 162 & $\mathrm{C}$ aromatic & $: \mathrm{C} 2 \mathrm{O}$ & 8.04 & 443 & 253 \\
\hline 163 & $\mathrm{C}$ aromatic & $: \mathrm{C} 2 \mathrm{~S}$ & 9.47 & 30 & 25 \\
\hline 164 & $\mathrm{C}$ aromatic & :C2Si & 4.67 & 10 & 8 \\
\hline 165 & C aromatic & $: \mathrm{C} 2 \mathrm{~F}$ & 4.45 & 143 & 72 \\
\hline 166 & C aromatic & $: \mathrm{C} 2 \mathrm{Cl}$ & 9.43 & 429 & 146 \\
\hline 167 & C aromatic & $: \mathrm{C} 2 \mathrm{Br}$ & 12.49 & 149 & 69 \\
\hline 168 & $\mathrm{C}$ aromatic & :C2J & 19.48 & 29 & 26 \\
\hline 169 & C aromatic & :CN:N & 16.72 & 2 & 2 \\
\hline 170 & C aromatic & :C:NO & 13.67 & 4 & 3 \\
\hline 171 & C aromatic & :C:NF & 14.34 & 1 & 1 \\
\hline 172 & $\mathrm{C}$ aromatic & :C:NCl & 15.74 & 3 & 3 \\
\hline 173 & C aromatic & $: \mathrm{C}: \mathrm{NBr}$ & 25.24 & 1 & 1 \\
\hline 174 & $\mathrm{C}$ aromatic & $\mathrm{N}: \mathrm{N} 2$ & 20.19 & 5 & 2 \\
\hline 175 & C aromatic & :N2O & 16.44 & 2 & 2 \\
\hline 176 & C sp & $\mathrm{H \# C}$ & 2.42 & 15 & 14 \\
\hline 177 & C sp & $\mathrm{C \# C}$ & 6.05 & 62 & 33 \\
\hline 178 & C sp & $=\mathrm{C} 2$ & 5.50 & 4 & 4 \\
\hline
\end{tabular}


Table 1. Cont.

\begin{tabular}{|c|c|c|c|c|c|}
\hline Entry & Atom Type & Neighbours & Contribution & Occurrences & Molecules \\
\hline 179 & $\mathrm{Csp}$ & $\mathrm{CHN}$ & 17.38 & 72 & 70 \\
\hline 180 & C sp & $\# \mathrm{CCl}$ & 9.31 & 3 & 2 \\
\hline 181 & C sp & $=\mathrm{N}=\mathrm{O}$ & 10.44 & 6 & 5 \\
\hline 182 & $C \mathrm{sp}$ & $=\mathrm{N}=\mathrm{S}$ & 23.08 & 3 & 3 \\
\hline 183 & $\mathrm{~N} \mathrm{sp}{ }^{3}$ & $\mathrm{H} 2 \mathrm{C}$ & 2.30 & 78 & 58 \\
\hline 184 & $\mathrm{~N} \mathrm{sp}^{3}$ & $\mathrm{H} 2 \mathrm{C}(\mathrm{pi})$ & 8.05 & 61 & 59 \\
\hline 185 & $\mathrm{~N} \mathrm{sp}^{3}$ & $\mathrm{H} 2 \mathrm{~N}$ & 19.23 & 8 & 7 \\
\hline 186 & $\mathrm{~N} \mathrm{sp}^{3}$ & $\mathrm{H} 2 \mathrm{~S}$ & 28.18 & 2 & 2 \\
\hline 187 & $\mathrm{~N} \mathrm{sp}^{3}$ & $\mathrm{HC} 2$ & -11.34 & 59 & 56 \\
\hline 188 & $\mathrm{~N} \mathrm{sp}^{3}$ & HC2(pi) & -1.94 & 27 & 26 \\
\hline 189 & $\mathrm{~N} \mathrm{sp}^{3}$ & HC2(2pi) & -2.43 & 21 & 21 \\
\hline 190 & $\mathrm{~N} \mathrm{sp}^{3}$ & $\mathrm{HCN}$ & -0.76 & 3 & 2 \\
\hline 191 & $\mathrm{~N} \mathrm{sp}^{3}$ & $\mathrm{HCN}(\mathrm{pi})$ & -13.33 & 3 & 3 \\
\hline 192 & $\mathrm{~N} \mathrm{sp}^{3}$ & $\operatorname{HCN}(2 \mathrm{pi})$ & 4.97 & 1 & 1 \\
\hline 193 & $\mathrm{~N} \mathrm{sp}^{3}$ & HCS(pi) & 5.34 & 7 & 7 \\
\hline 194 & $\mathrm{~N} \mathrm{sp}^{3}$ & $\mathrm{HCSi}$ & -4.02 & 6 & 6 \\
\hline 195 & $\mathrm{~N} \mathrm{sp}^{3}$ & HSi2 & 1.94 & 1 & 1 \\
\hline 196 & $\mathrm{~N} \mathrm{sp}^{3}$ & $\mathrm{BC} 2$ & -31.30 & 3 & 2 \\
\hline 197 & $\mathrm{~N} \mathrm{sp}^{3}$ & $\mathrm{C} 3$ & -30.50 & 111 & 101 \\
\hline 198 & $\mathrm{~N} \mathrm{sp}^{3}$ & C3(pi) & -25.56 & 37 & 31 \\
\hline 199 & $\mathrm{~N} \mathrm{sp}^{3}$ & C3(2pi) & -22.95 & 52 & 50 \\
\hline 200 & $\mathrm{~N} \mathrm{sp}^{3}$ & C3(3pi) & -27.03 & 13 & 13 \\
\hline 201 & $\mathrm{~N} \mathrm{sp}^{3}$ & $\mathrm{C} 2 \mathrm{~N}$ & -19.64 & 4 & 3 \\
\hline 202 & $\mathrm{~N} \mathrm{sp}^{3}$ & $\mathrm{C} 2 \mathrm{~N}(+)$ & 0.00 & 1 & 1 \\
\hline 203 & $\mathrm{~N} \mathrm{sp}^{3}$ & $\mathrm{C} 2 \mathrm{~N}(\mathrm{pi})$ & -27.16 & 3 & 2 \\
\hline 204 & $\mathrm{~N} \mathrm{sp}^{3}$ & $\mathrm{C} 2 \mathrm{~N}(+)(\mathrm{pi})$ & 3.24 & 4 & 4 \\
\hline 205 & $\mathrm{~N} \mathrm{sp}^{3}$ & $\mathrm{C} 2 \mathrm{~N}(2 \mathrm{pi})$ & -24.28 & 4 & 4 \\
\hline 206 & $\mathrm{~N} \mathrm{sp}^{3}$ & C2N(3pi) & -26.84 & 2 & 2 \\
\hline 207 & $\mathrm{~N} \mathrm{sp}^{3}$ & $\mathrm{C} 2 \mathrm{O}$ & 8.24 & 1 & 1 \\
\hline 208 & $\mathrm{~N} \mathrm{sp}^{3}$ & $\mathrm{C} 2 \mathrm{P}$ & -17.98 & 5 & 2 \\
\hline 209 & $\mathrm{~N} \mathrm{sp}{ }^{3}$ & $\mathrm{C} 2 \mathrm{Si}$ & -19.79 & 12 & 8 \\
\hline 210 & $\mathrm{Nsp}^{3}$ & CN2(2pi) & -36.43 & 1 & 1 \\
\hline 211 & $\mathrm{~N} \mathrm{sp}^{3}$ & $\mathrm{CN} 2(+)(2 \mathrm{pi})$ & 16.44 & 1 & 1 \\
\hline 212 & $\mathrm{~N} \mathrm{sp}^{3}$ & CF2 & -4.56 & 2 & 2 \\
\hline 213 & $\mathrm{~N} \mathrm{sp}^{3}$ & CF2(pi) & -12.61 & 1 & 1 \\
\hline 214 & $\mathrm{~N} \mathrm{sp}^{3}$ & $\mathrm{CSi} 2$ & -17.81 & 1 & 1 \\
\hline 215 & $\mathrm{~N} \mathrm{sp}^{3}$ & $\mathrm{Si} 3$ & -1.79 & 1 & 1 \\
\hline 216 & $\mathrm{~N} \mathrm{sp}^{2}$ & $\mathrm{H}=\mathrm{C}$ & 1.29 & 2 & 2 \\
\hline 217 & $\mathrm{~N} \mathrm{sp}^{2}$ & $\mathrm{C}=\mathrm{C}$ & -10.46 & 85 & 82 \\
\hline 218 & $\mathrm{~N} \mathrm{sp}^{2}$ & $\mathrm{C}=\mathrm{N}$ & -5.89 & 19 & 10 \\
\hline 219 & $\mathrm{~N} \mathrm{sp}^{2}$ & $\mathrm{C}=\mathrm{N}(+)$ & -2.79 & 15 & 13 \\
\hline 220 & $\mathrm{~N} \mathrm{sp}^{2}$ & $=\mathrm{CN}$ & 18.81 & 9 & 9 \\
\hline 221 & $\mathrm{~N} \mathrm{sp}^{2}$ & $=\mathrm{CO}$ & 10.27 & 17 & 14 \\
\hline 222 & $\mathrm{~N} \mathrm{sp}^{2}$ & $=\mathrm{CF}$ & 0.00 & 1 & 1 \\
\hline 223 & $\mathrm{~N} \mathrm{sp}^{2}$ & $\mathrm{~N}=\mathrm{N}$ & 15.91 & 5 & 3 \\
\hline 224 & $\mathrm{~N} \mathrm{sp}^{2}$ & $\mathrm{O}=\mathrm{O}$ & 0.59 & 7 & 7 \\
\hline 225 & $\mathrm{~N}$ aromatic & $: \mathrm{C} 2$ & -5.10 & 104 & 78 \\
\hline 226 & $\mathrm{~N}$ aromatic & $: \mathrm{C}: \mathrm{N}$ & 5.35 & 8 & 4 \\
\hline 227 & $\mathrm{~N}(+) \mathrm{sp}^{3}$ & $\mathrm{C} 2 \mathrm{NO}(-)$ & 0.00 & 1 & 1 \\
\hline 228 & $\mathrm{~N}(+) \mathrm{sp}^{2}$ & $\mathrm{CO}=\mathrm{O}(-)$ & -2.09 & 78 & 56 \\
\hline 229 & $\mathrm{~N}(+) \mathrm{sp}^{2}$ & $\mathrm{C}=\mathrm{NO}(-)$ & -19.89 & 3 & 3 \\
\hline 230 & $\mathrm{~N}(+) \mathrm{sp}^{2}$ & $\mathrm{NO}=\mathrm{O}(-)$ & 0.35 & 6 & 5 \\
\hline 231 & $\mathrm{~N}(+) \mathrm{sp}^{2}$ & $\mathrm{O} 2=\mathrm{O}(-)$ & 9.02 & 17 & 11 \\
\hline
\end{tabular}


Table 1. Cont.

\begin{tabular}{|c|c|c|c|c|c|}
\hline Entry & Atom Type & Neighbours & Contribution & Occurrences & Molecules \\
\hline 232 & $\mathrm{~N}(+)$ aromatic & :C2O(-) & 0.00 & 1 & 1 \\
\hline 233 & $\mathrm{~N}(+) \mathrm{sp}$ & $\mathrm{C \# C}(-)$ & -8.48 & 2 & 2 \\
\hline 234 & $\mathrm{~N}(+) \mathrm{sp}$ & $=\mathrm{N} 2(-)$ & 5.96 & 12 & 10 \\
\hline 235 & $\mathrm{O}^{1}$ & $\mathrm{HC}$ & 14.55 & 322 & 288 \\
\hline 236 & $\mathrm{O}$ & $\mathrm{HC}(\mathrm{pi})$ & 20.98 & 174 & 157 \\
\hline 237 & $\mathrm{O}$ & $\mathrm{HN}$ & 0.00 & 1 & 1 \\
\hline 238 & $\mathrm{O}$ & $\mathrm{HN}(\mathrm{pi})$ & 19.03 & 2 & 2 \\
\hline 239 & $\mathrm{O}$ & $\mathrm{HO}$ & 23.75 & 5 & 5 \\
\hline 240 & $\mathrm{O}$ & HSi & 26.41 & 1 & 1 \\
\hline 241 & $\mathrm{O}$ & $\mathrm{BC}$ & -17.91 & 5 & 3 \\
\hline 242 & $\mathrm{O}$ & $\mathrm{C} 2$ & -17.86 & 424 & 270 \\
\hline 243 & $\mathrm{O}$ & C2(pi) & -13.29 & 744 & 629 \\
\hline 244 & $\mathrm{O}$ & C2(2pi) & -7.15 & 145 & 120 \\
\hline 245 & $\mathrm{O}$ & $\mathrm{CN}(\mathrm{pi})$ & 0.00 & 7 & 7 \\
\hline 246 & $\mathrm{O}$ & $\mathrm{CN}(+)(\mathrm{pi})$ & 2.17 & 17 & 11 \\
\hline 247 & $\mathrm{O}$ & $\mathrm{CN}(2 \mathrm{pi})$ & -2.82 & 9 & 9 \\
\hline 248 & $\mathrm{O}$ & $\mathrm{CO}$ & -8.76 & 54 & 20 \\
\hline 249 & $\mathrm{O}$ & CS & 2.45 & 18 & 9 \\
\hline 250 & $\mathrm{O}$ & $\mathrm{CP}$ & -2.71 & 104 & 42 \\
\hline 251 & $\mathrm{O}$ & $\mathrm{CP}(\mathrm{pi})$ & 1.25 & 7 & 5 \\
\hline 252 & $\mathrm{O}$ & $\mathrm{CSi}$ & -11.39 & 79 & 29 \\
\hline 253 & $\mathrm{O}$ & CSi(pi) & -14.85 & 37 & 13 \\
\hline 254 & $\mathrm{O}$ & N2(2pi) & -0.72 & 3 & 3 \\
\hline 255 & $\mathrm{O}$ & OSi & 4.23 & 9 & 4 \\
\hline 256 & $\mathrm{O}$ & $\mathrm{P} 2$ & 16.68 & 1 & 1 \\
\hline 257 & $\mathrm{O}$ & Si2 & -6.52 & 15 & 4 \\
\hline 258 & P3 & $\mathrm{C} 3$ & -6.83 & 3 & 3 \\
\hline 259 & P3 & $\mathrm{C} 2 \mathrm{O}$ & 2.71 & 1 & 1 \\
\hline 260 & P3 & N3 & -7.09 & 1 & 1 \\
\hline 261 & P3 & $\mathrm{N} 2 \mathrm{Cl}$ & 10.64 & 1 & 1 \\
\hline 262 & P3 & $\mathrm{O} 3$ & -4.07 & 1 & 1 \\
\hline 263 & P4 & $\mathrm{HO} 2=\mathrm{O}$ & 9.23 & 2 & 2 \\
\hline 264 & P4 & $\mathrm{CO} 2=\mathrm{O}$ & 5.40 & 3 & 3 \\
\hline 265 & $\mathrm{P} 4$ & $\mathrm{O} 3=\mathrm{O}$ & -3.86 & 16 & 15 \\
\hline 266 & P4 & $\mathrm{O} 3=\mathrm{S}$ & 1.10 & 9 & 9 \\
\hline 267 & P4 & $\mathrm{O} 2=\mathrm{OS}$ & 1.77 & 4 & 4 \\
\hline 268 & $\mathrm{P} 4$ & $\mathrm{O} 2 \mathrm{~S}=\mathrm{S}$ & 1.73 & 8 & 8 \\
\hline 269 & S2 & $\mathrm{HC}$ & 1.49 & 33 & 29 \\
\hline 270 & S2 & $\mathrm{HC}(\mathrm{pi})$ & 6.23 & 1 & 1 \\
\hline 271 & S2 & $\mathrm{HP}$ & 23.50 & 3 & 3 \\
\hline 272 & S2 & $\mathrm{BC}$ & -24.53 & 12 & 4 \\
\hline 273 & S2 & $\mathrm{C} 2$ & -10.51 & 67 & 65 \\
\hline 274 & S2 & C2(pi) & -2.71 & 23 & 22 \\
\hline 275 & S2 & C2(2pi) & 0.53 & 44 & 44 \\
\hline 276 & S2 & CS & -0.35 & 16 & 8 \\
\hline 277 & S2 & $\mathrm{CS}(\mathrm{pi})$ & 2.39 & 2 & 1 \\
\hline 278 & S2 & $\mathrm{CP}$ & -1.99 & 9 & 9 \\
\hline 279 & S2 & Si2 & -3.40 & 1 & 1 \\
\hline 280 & S4 & $\mathrm{C} 2=\mathrm{O}$ & 22.60 & 4 & 4 \\
\hline 281 & S4 & $\mathrm{C} 2=\mathrm{O} 2$ & 27.80 & 9 & 9 \\
\hline 282 & S4 & $\mathrm{C} 2 \mathrm{~F} 2$ & -5.92 & 1 & 1 \\
\hline 283 & S4 & $\mathrm{CN}=\mathrm{O} 2$ & 1.94 & 9 & 9 \\
\hline 284 & S4 & $\mathrm{C}=\mathrm{O} 2 \mathrm{~S}$ & 37.54 & 2 & 1 \\
\hline 285 & S4 & $\mathrm{O} 2=\mathrm{O}$ & -3.83 & 5 & 5 \\
\hline 286 & S4 & $\mathrm{O} 2=\mathrm{O} 2$ & 4.79 & 4 & 4 \\
\hline 287 & $\mathrm{Si}$ & $\mathrm{H} 3 \mathrm{C}$ & 0.00 & 1 & 1 \\
\hline
\end{tabular}


Table 1. Cont.

\begin{tabular}{|c|c|c|c|c|c|}
\hline Entry & Atom Type & Neighbours & Contribution & Occurrences & Molecules \\
\hline 288 & $\mathrm{Si}$ & $\mathrm{H} 2 \mathrm{CN}$ & 2.20 & 1 & 1 \\
\hline 289 & $\mathrm{Si}$ & HC3 & -4.21 & 24 & 24 \\
\hline 290 & $\mathrm{Si}$ & $\mathrm{HC} 2 \mathrm{O}$ & 2.36 & 2 & 1 \\
\hline 291 & $\mathrm{Si}$ & $\mathrm{HC} 2 \mathrm{~S}$ & 0.00 & 2 & 1 \\
\hline 292 & $\mathrm{Si}$ & $\mathrm{HCO} 2$ & 8.33 & 5 & 1 \\
\hline 293 & $\mathrm{Si}$ & HN3 & 8.01 & 2 & 2 \\
\hline 294 & $\mathrm{Si}$ & $\mathrm{C} 4$ & -0.57 & 21 & 20 \\
\hline 295 & $\mathrm{Si}$ & $\mathrm{C} 3 \mathrm{~N}$ & -1.80 & 18 & 14 \\
\hline 296 & $\mathrm{Si}$ & $\mathrm{C} 3 \mathrm{O}$ & 0.35 & 6 & 6 \\
\hline 297 & $\mathrm{Si}$ & $\mathrm{C} 2 \mathrm{O} 2$ & 5.64 & 18 & 11 \\
\hline 298 & $\mathrm{Si}$ & $\mathrm{CO} 3$ & -2.40 & 26 & 26 \\
\hline 299 & $\mathrm{Si}$ & O4 & -16.14 & 6 & 6 \\
\hline 300 & $\mathrm{H}$ & H Acceptor & -12.45 & 16 & 16 \\
\hline 301 & Alkane & No. of $C$ atoms & 0.09 & 3072 & 286 \\
\hline 302 & Unsaturated HC & No. of $C$ atoms & -0.07 & 4100 & 413 \\
\hline A & Based on & Valid groups & 185 & & 3581 \\
\hline $\mathrm{B}$ & Goodness of fit & $R^{2}$ & 0.9678 & & 3460 \\
\hline $\mathrm{C}$ & Deviation & Average & 2.99 & & 3460 \\
\hline $\mathrm{D}$ & Deviation & Standard & 4.30 & & 3460 \\
\hline $\mathrm{E}$ & K-fold cv & K & 10 & & 3386 \\
\hline $\mathrm{F}$ & Goodness of fit & $Q^{2}$ & 0.9641 & & 3386 \\
\hline G & Deviation & Average (cv) & 3.14 & & 3386 \\
\hline $\mathrm{H}$ & Deviation & Standard (cv) & 4.56 & & 3386 \\
\hline
\end{tabular}

The total number of atom groups in Table 1, required to take account of the complete set of 3581 molecules for which experimental vaporization data are known, is 302 . However, the condition to restrict their applicability to those resting on at least three independent molecules, reduces the number of "valid groups" to 187, as is shown in row A of Table 1. Accordingly, the number of compounds viable for the evaluation of the result of the complete training set and of the test sets in the 10-fold cross-validation calculation was reduced to 3460 and 3381, respectively, as listed in the right-most column. The high correlation coefficients $R^{2}$ and $Q^{2}$ of the training and the cross-validated sets (rows $\mathrm{B}$ and F) of better than 0.96 and the small difference between them is clear proof of the viability of the present group-additivity model for the prediction of the enthalpy of vaporization. Furthermore, the small standard deviations for the training and test sets of 4.3 and 4.56 (rows D and $\mathrm{H}$ ) also speaks for the model's accuracy. In order to put these deviations into perspective with the reality of the experimental practice, a few examples should be given for comparison: the compilation of Acree and Chickos [8] presented eight values for 1-butanol ranging from 48.4 to $55.2 \mathrm{~kJ} / \mathrm{mol}$, seven values for methyl $t$-amyl ether ranging from 33.5 to $35.8 \mathrm{~kJ} / \mathrm{mol}$, and four values for ethylenediamine of between 41 and $54.4 \mathrm{~kJ} / \mathrm{mol}$. It goes without saying, therefore, that the standard errors of the group-parameters calculations (lines $\mathrm{D}$ and $\mathrm{H}$ in the parameters table), covering the complete set of available data, are always larger than the individual errors and, thus better reflect the general uncertainty of the experimental data.

The correlation diagram in Figure 1, showing a fairly even distribution of the vaporization data along the regression line, also reveals a narrow overlap of the cross-validated test data with those of the training set. The related histogram in Figure 2, exhibiting a nearly perfect Gaussian bell curve, proves the evenness of the distribution of the deviations of both test and training data about the regression line. The analysis of the distribution of the deviations yielded the following result: $79.2 \%$ of the presently 3460 tested compounds deviated by less than or equal to one cross-validated standard error of $4.56 \mathrm{~kJ} / \mathrm{mol}$, whereas $6.8 \%$ exceeded a deviation of twice that standard error. Beyond this, 32 molecules had to be viewed as outliers as their deviation surpassed by at least four times this standard deviation. 


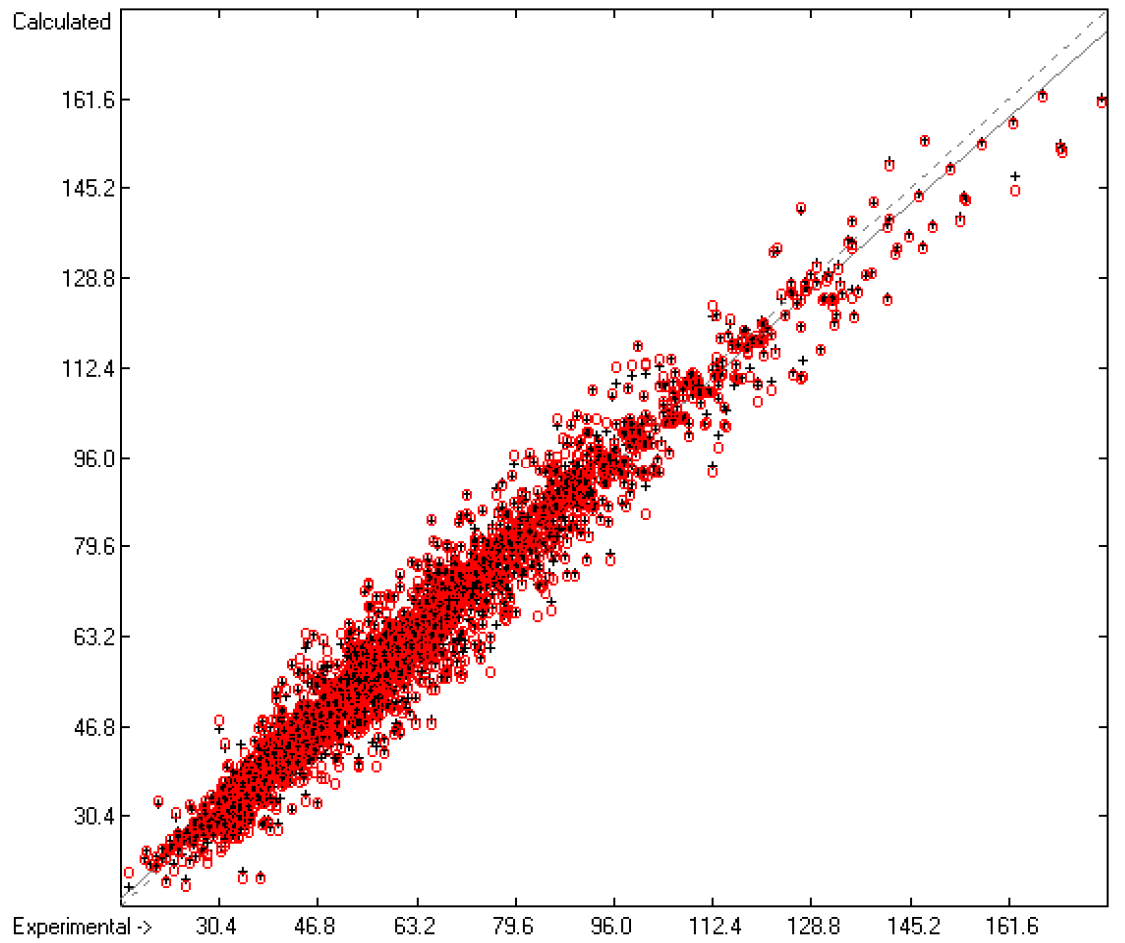

Figure 1. Correlation Diagram of the Enthalpy-of-Vaporization Data $\left(N=3460 ; R^{2}=0.9677 ; Q^{2}=0.9640\right.$; regression line: intercept $=1.9756$, slope $=0.9681$ ).

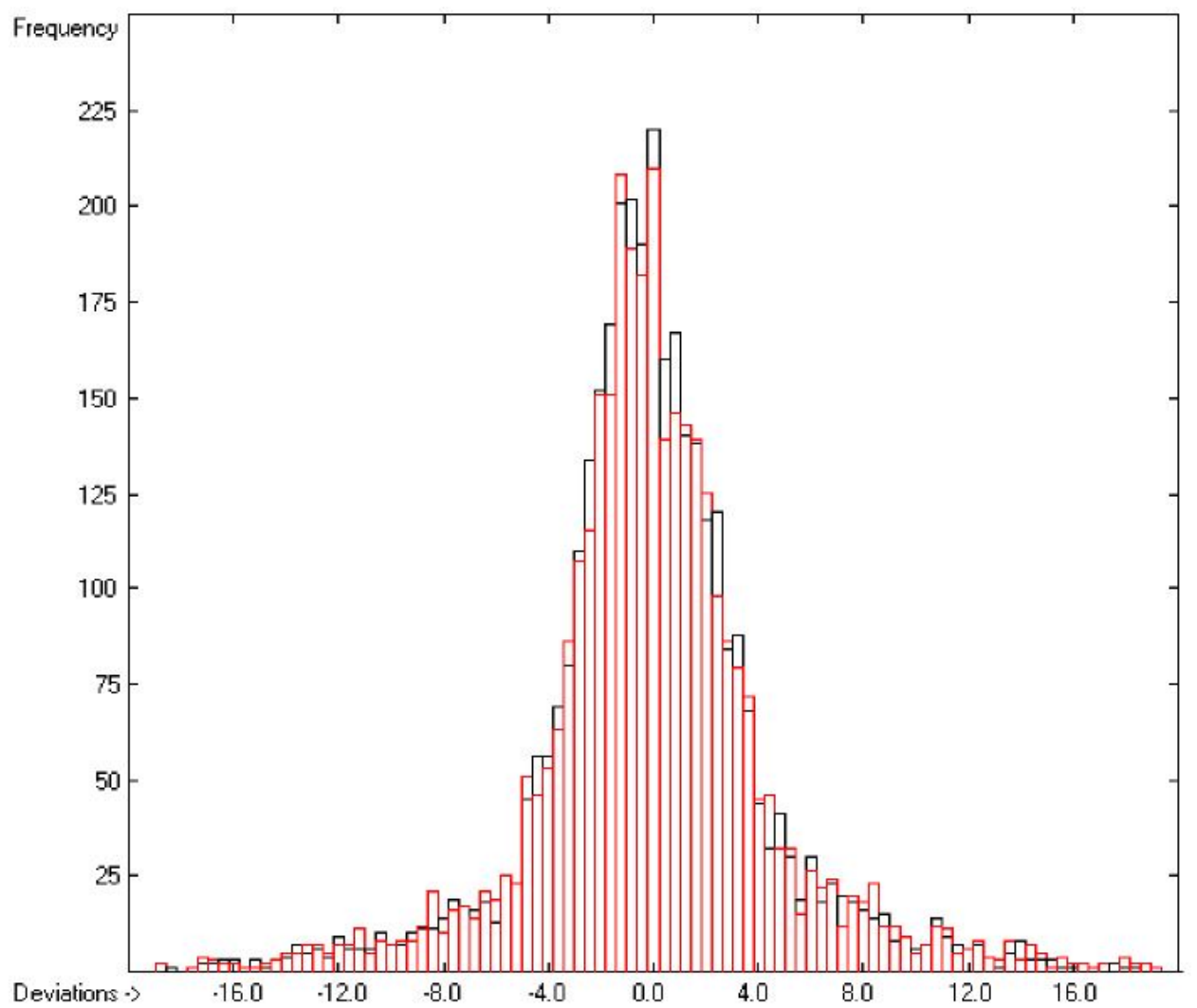

Figure 2. Histogram of the Enthalpy-of-Vaporization Data $(S=4.56 \mathrm{~kJ} / \mathrm{mol}$; Exp. values range: $15.6-177.2 \mathrm{~kJ} / \mathrm{mol})$. 
Despite the detailed distinction of the atom groups in Table 1, resulting in an extended list of groups of which about one third is "invalid", the still large number of "valid" atom groups enabled the calculation of reliable enthalpy-of-vaporization data for $78.2 \%$ of the complete set of compounds in the database.

\subsection{Enthalpy of Sublimation}

The enthalpy of sublimation is the sum of the enthalpies of vaporization and fusion, provided that all of them are referenced to the same temperature. This precondition has been thoroughly followed when selecting experimental data from literature. Again, as in the previous section, the main contribution of experimental sublimation values has been provided by the compendium of Acree, Jr. et al. [8], supplemented by a number of later publications, referencing the heat of sublimation of acetophenones [23], substituted benzamides [26], crown ethers [29], long-chain fluorinated alcohols [33], halogenated fluorenes [35], tricyclic nitrogen heteroaromatics [42], polyphenylbenzenes [47], adamantylideneadamantane [48], cyclic $N, N^{\prime}$-thioureas [49], indole-3-carboxylic acids [50], vanillyl alcohol [51], alkanoylphenols [52], adamantanes [53], six-membered ring aliphatics [54], fluoroquinolones [55], oxazolidinones [56], nitrogen-containing substituted adamantanes [57], 2,7-di-t-butylfluorene [58] and nitroimidazoles [59].

Table 2. Atom Groups and their Contributions (in kJ/mol) for Heat-of-Sublimation Calculations.

\begin{tabular}{|c|c|c|c|c|c|}
\hline Entry & Atom Type & Neighbours & Contribution & Occurrences & Molecules \\
\hline 1 & Const & & 21.03 & 1960 & 1960 \\
\hline 2 & B & $\mathrm{C} 3$ & 65.82 & 2 & 2 \\
\hline 3 & $\mathrm{Csp} \mathrm{sp}^{3}$ & $\mathrm{H} 3 \mathrm{C}$ & 5.99 & 1322 & 623 \\
\hline 4 & $\mathrm{C} \mathrm{sp}^{3}$ & $\mathrm{H} 3 \mathrm{~N}$ & 26.96 & 143 & 87 \\
\hline 5 & $\mathrm{C} \mathrm{sp}^{3}$ & $\mathrm{H} 3 \mathrm{~N}(+)$ & 98.98 & 1 & 1 \\
\hline 6 & $\mathrm{Csp} \mathrm{sp}^{3}$ & $\mathrm{H} 3 \mathrm{O}$ & 28.51 & 181 & 122 \\
\hline 7 & $C \mathrm{sp}^{3}$ & $\mathrm{H} 3 \mathrm{~S}$ & 30.06 & 7 & 6 \\
\hline 8 & $C \mathrm{sp}^{3}$ & $\mathrm{H} 2 \mathrm{C} 2$ & 6.88 & 2602 & 508 \\
\hline 9 & $\mathrm{Csp} \mathrm{sp}^{3}$ & $\mathrm{H} 2 \mathrm{CN}$ & 21.98 & 224 & 116 \\
\hline 10 & $C \mathrm{sp}^{3}$ & $\mathrm{H} 2 \mathrm{CN}(+)$ & 27.46 & 13 & 11 \\
\hline 11 & $C \mathrm{sp}^{3}$ & $\mathrm{H} 2 \mathrm{CO}$ & 29.62 & 242 & 134 \\
\hline 12 & $C \mathrm{sp}^{3}$ & $\mathrm{H} 2 \mathrm{CS}$ & 23.29 & 50 & 31 \\
\hline 13 & $\mathrm{Csp}$ & $\mathrm{H} 2 \mathrm{CF}$ & 15.91 & 1 & 1 \\
\hline 14 & $C \mathrm{sp}^{3}$ & $\mathrm{H} 2 \mathrm{CCl}$ & 17.59 & 3 & 3 \\
\hline 15 & $C \mathrm{sp}^{3}$ & $\mathrm{H} 2 \mathrm{CBr}$ & 22.76 & 5 & 4 \\
\hline 16 & $C \mathrm{sp}^{3}$ & $\mathrm{H} 2 \mathrm{CJ}$ & 21.83 & 3 & 2 \\
\hline 17 & $\mathrm{Csp} \mathrm{sp}^{3}$ & $\mathrm{H} 2 \mathrm{~N} 2$ & 43.95 & 18 & 6 \\
\hline 18 & $\mathrm{Csp} \mathrm{sp}^{3}$ & $\mathrm{H} 2 \mathrm{NCl}$ & 36.29 & 1 & 1 \\
\hline 19 & $C \mathrm{sp}^{3}$ & $\mathrm{H} 2 \mathrm{O} 2$ & 53.35 & 25 & 13 \\
\hline 20 & $C \mathrm{sp}^{3}$ & $\mathrm{H} 2 \mathrm{OS}$ & 54.78 & 1 & 1 \\
\hline 21 & $\mathrm{C} \mathrm{sp}^{3}$ & $\mathrm{H} 2 \mathrm{~S} 2$ & 47.45 & 6 & 4 \\
\hline 22 & $\mathrm{Csp} \mathrm{sp}^{3}$ & HBC2 & -36.17 & 3 & 1 \\
\hline 23 & $\mathrm{Csp} \mathrm{sp}^{3}$ & $\mathrm{HC} 3$ & 2.28 & 509 & 190 \\
\hline 24 & $C \mathrm{sp}^{3}$ & $\mathrm{HC} 2 \mathrm{~N}$ & 14.28 & 34 & 30 \\
\hline 25 & $\mathrm{Csp} \mathrm{sp}^{3}$ & $\mathrm{HC} 2 \mathrm{~N}(+)$ & 21.01 & 9 & 9 \\
\hline 26 & $\mathrm{Csp} \mathrm{sp}^{3}$ & $\mathrm{HC} 2 \mathrm{O}$ & 24.27 & 82 & 47 \\
\hline 27 & $C \mathrm{sp}^{3}$ & $\mathrm{HC} 2 \mathrm{~S}$ & 17.59 & 14 & 11 \\
\hline 28 & $C \mathrm{sp}^{3}$ & $\mathrm{HC} 2 \mathrm{~F}$ & 5.18 & 1 & 1 \\
\hline 29 & $\mathrm{Csp} \mathrm{sp}^{3}$ & $\mathrm{HC} 2 \mathrm{Cl}$ & 11.49 & 7 & 2 \\
\hline 30 & $C \mathrm{sp}^{3}$ & $\mathrm{HC} 2 \mathrm{Br}$ & -0.95 & 1 & 1 \\
\hline 31 & $C \mathrm{sp}^{3}$ & $\mathrm{HCN} 2$ & 39.48 & 8 & 2 \\
\hline 32 & $\mathrm{C} \mathrm{sp}^{3}$ & $\mathrm{HCN} 2(+)$ & 39.93 & 2 & 2 \\
\hline 33 & $C \mathrm{sp}^{3}$ & HCNO & 34.73 & 2 & 1 \\
\hline
\end{tabular}


Table 2. Cont.

\begin{tabular}{|c|c|c|c|c|c|}
\hline Entry & Atom Type & Neighbours & Contribution & Occurrences & Molecules \\
\hline 34 & $\mathrm{Csp}^{3}$ & HCNS & 20.56 & 2 & 1 \\
\hline 35 & $\mathrm{Csp}$ & $\mathrm{HCO} 2$ & 39.96 & 3 & 3 \\
\hline 36 & $\mathrm{Csp}$ & HCF2 & -0.19 & 1 & 1 \\
\hline 37 & $\mathrm{Csp}^{3}$ & $\mathrm{HCCl} 2$ & 15.78 & 1 & 1 \\
\hline 38 & $\mathrm{Csp}$ & $\mathrm{HN} 3(+)$ & 37.31 & 1 & 1 \\
\hline 39 & $\mathrm{Csp}^{3}$ & $\mathrm{HO} 3$ & 72.23 & 3 & 3 \\
\hline 40 & $\mathrm{Csp}$ & $\mathrm{C} 4$ & -4.25 & 209 & 137 \\
\hline 41 & $\mathrm{Csp}$ & $\mathrm{C} 3 \mathrm{~N}$ & 5.87 & 18 & 13 \\
\hline 42 & $\mathrm{Csp}$ & $\mathrm{C} 3 \mathrm{~N}(+)$ & 18.44 & 14 & 11 \\
\hline 43 & $\mathrm{Csp}$ & $\mathrm{C} 3 \mathrm{O}$ & 15.18 & 40 & 31 \\
\hline 44 & $\mathrm{Csp}$ & C3S & 6.40 & 5 & 5 \\
\hline 45 & $\mathrm{Csp}^{3}$ & $\mathrm{C} 3 \mathrm{~F}$ & 1.89 & 3 & 3 \\
\hline 46 & $\mathrm{Csp}$ & $\mathrm{C} 3 \mathrm{Cl}$ & -8.06 & 1 & 1 \\
\hline 47 & $\mathrm{Csp}$ & $\mathrm{C} 3 \mathrm{Br}$ & 2.34 & 1 & 1 \\
\hline 48 & $\mathrm{Csp}$ & $\mathrm{C} 2 \mathrm{~N} 2(+)$ & 34.78 & 7 & 6 \\
\hline 49 & $\mathrm{Csp}$ & $\mathrm{C} 2 \mathrm{O} 2$ & 39.73 & 8 & 8 \\
\hline 50 & $\mathrm{C} \mathrm{sp}^{3}$ & $\mathrm{C} 2 \mathrm{~S} 2$ & 37.28 & 4 & 1 \\
\hline 51 & $\mathrm{Csp}$ & $\mathrm{C} 2 \mathrm{~F} 2$ & 7.07 & 62 & 8 \\
\hline 52 & $\mathrm{Csp}$ & $\mathrm{CN} 3(+)$ & 43.89 & 19 & 12 \\
\hline 53 & $\mathrm{Csp}^{3}$ & $\mathrm{CN} 2 \mathrm{~F}(+)$ & 25.98 & 1 & 1 \\
\hline 54 & $\mathrm{Csp}$ & $\mathrm{CO} 3$ & 57.42 & 2 & 2 \\
\hline 55 & $\mathrm{Csp}$ & CF3 & -4.71 & 27 & 23 \\
\hline 56 & $\mathrm{C} \mathrm{sp}^{3}$ & $\mathrm{CCl} 3$ & 16.10 & 3 & 2 \\
\hline 57 & $\mathrm{Csp}$ & $\mathrm{N} 3 \mathrm{~F}(+)$ & 44.00 & 1 & 1 \\
\hline 58 & $\mathrm{Csp}^{3}$ & $\mathrm{O} 4$ & 73.43 & 1 & 1 \\
\hline 59 & $\mathrm{Csp} \mathrm{sp}^{2}$ & $\mathrm{H} 2=\mathrm{C}$ & 7.97 & 12 & 12 \\
\hline 60 & $\mathrm{Csp} \mathrm{sp}^{2}$ & $\mathrm{HC}=\mathrm{C}$ & 5.10 & 452 & 213 \\
\hline 61 & $\mathrm{Csp}^{2}$ & $\mathrm{HC}=\mathrm{N}$ & 35.49 & 21 & 19 \\
\hline 62 & $\mathrm{Csp}{ }^{2}$ & $\mathrm{HC}=\mathrm{N}(+)$ & 72.64 & 7 & 7 \\
\hline 63 & $\mathrm{C} \mathrm{sp}^{2}$ & $\mathrm{H}=\mathrm{CN}$ & 32.79 & 83 & 69 \\
\hline 64 & $\mathrm{C} \mathrm{sp}^{2}$ & $\mathrm{HC}=\mathrm{O}$ & 20.74 & 15 & 15 \\
\hline 65 & $\mathrm{Csp}^{2}$ & $\mathrm{H}=\mathrm{CO}$ & 16.89 & 16 & 14 \\
\hline 66 & $\mathrm{C} \mathrm{sp}^{2}$ & $\mathrm{H}=\mathrm{CS}$ & 15.22 & 49 & 36 \\
\hline 67 & $\mathrm{Csp} \mathrm{sp}^{2}$ & $\mathrm{HN}=\mathrm{N}$ & 55.52 & 19 & 18 \\
\hline 68 & $\mathrm{Csp}^{2}$ & $\mathrm{HN}=\mathrm{O}$ & 35.41 & 4 & 3 \\
\hline 69 & $\mathrm{C} \mathrm{sp}^{2}$ & $\mathrm{H}=\mathrm{NO}$ & 40.91 & 1 & 1 \\
\hline 70 & $\mathrm{Csp}^{2}$ & $\mathrm{H}=\mathrm{NS}$ & 33.85 & 2 & 2 \\
\hline 71 & $\mathrm{Csp}^{2}$ & $\mathrm{C} 2=\mathrm{C}$ & 3.91 & 78 & 61 \\
\hline 72 & $\mathrm{Csp}$ & $\mathrm{C} 2=\mathrm{N}$ & 30.47 & 35 & 26 \\
\hline 73 & $\mathrm{Csp} \mathrm{sp}^{2}$ & $\mathrm{C} 2=\mathrm{N}(+)$ & 13.76 & 5 & 5 \\
\hline 74 & $\mathrm{Csp}^{2}$ & $\mathrm{C}=\mathrm{CN}$ & 26.81 & 57 & 48 \\
\hline 75 & $\mathrm{Csp}^{2}$ & $\mathrm{C}=\mathrm{CN}(+)$ & 41.65 & 7 & 7 \\
\hline 76 & $\mathrm{Csp} \mathrm{sp}^{2}$ & $\mathrm{C} 2=\mathrm{O}$ & 15.10 & 200 & 161 \\
\hline 77 & $\mathrm{Csp}^{2}$ & $\mathrm{C}=\mathrm{CO}$ & 22.08 & 40 & 31 \\
\hline 78 & $\mathrm{Csp} \mathrm{sp}^{2}$ & $\mathrm{C} 2=\mathrm{S}$ & 18.21 & 3 & 3 \\
\hline 79 & $\mathrm{C} \mathrm{sp}^{2}$ & $\mathrm{C}=\mathrm{CS}$ & 15.64 & 36 & 27 \\
\hline 80 & $\mathrm{C} \mathrm{sp}^{2}$ & $\mathrm{C}=\mathrm{CF}$ & 16.81 & 2 & 2 \\
\hline 81 & $\mathrm{Csp} \mathrm{sp}^{2}$ & $\mathrm{C}=\mathrm{CCl}$ & 11.02 & 9 & 5 \\
\hline 82 & $\mathrm{C} \mathrm{sp}^{2}$ & $\mathrm{C}=\mathrm{CBr}$ & 34.06 & 2 & 2 \\
\hline 83 & $\mathrm{Csp}^{2}$ & $\mathrm{C}=\mathrm{CJ}$ & 32.46 & 1 & 1 \\
\hline 84 & $\mathrm{Csp} \mathrm{sp}^{2}$ & $=\mathrm{CN} 2$ & 64.94 & 6 & 6 \\
\hline 85 & $\mathrm{C} \mathrm{sp}^{2}$ & $=\mathrm{CN} 2(+)$ & 60.65 & 4 & 4 \\
\hline 86 & $\mathrm{Csp}^{2}$ & $\mathrm{CN}=\mathrm{N}$ & 54.51 & 27 & 25 \\
\hline 87 & $\mathrm{C} \mathrm{sp}^{2}$ & $\mathrm{CN}=\mathrm{N}(+)$ & 44.16 & 3 & 3 \\
\hline 88 & $\mathrm{Csp} \mathrm{sp}^{2}$ & $\mathrm{CN}=\mathrm{O}$ & 39.66 & 234 & 194 \\
\hline
\end{tabular}


Table 2. Cont.

\begin{tabular}{|c|c|c|c|c|c|}
\hline Entry & Atom Type & Neighbours & Contribution & Occurrences & Molecules \\
\hline 89 & $\mathrm{Csp} \mathrm{sp}^{2}$ & $\mathrm{C}=\mathrm{NO}$ & 42.74 & 2 & 2 \\
\hline 90 & $\mathrm{Csp} \mathrm{sp}^{2}$ & $\mathrm{CN}=\mathrm{S}$ & 39.85 & 8 & 7 \\
\hline 91 & $\mathrm{Csp}^{2}$ & $\mathrm{C}=\mathrm{NS}$ & 34.89 & 1 & 1 \\
\hline 92 & $\mathrm{Csp}^{2}$ & $=\mathrm{CNS}(+)$ & 41.29 & 2 & 2 \\
\hline 93 & $\mathrm{Csp} \mathrm{sp}^{2}$ & $=\mathrm{CNCl}$ & 38.14 & 4 & 3 \\
\hline 94 & $\mathrm{Csp}^{2}$ & $\mathrm{CO}=\mathrm{O}$ & 34.06 & 424 & 345 \\
\hline 95 & $\mathrm{Csp} \mathrm{sp}^{2}$ & $\mathrm{CO}=\mathrm{O}(-)$ & 80.89 & 22 & 22 \\
\hline 96 & $\mathrm{Csp} \mathrm{sp}^{2}$ & $\mathrm{C}=\mathrm{OCl}$ & 29.03 & 1 & 1 \\
\hline 97 & $\mathrm{Csp}^{2}$ & $\mathrm{CS}=\mathrm{S}$ & 56.97 & 3 & 3 \\
\hline 98 & $\mathrm{Csp} \mathrm{sp}^{2}$ & $\mathrm{~N} 2=\mathrm{N}$ & 80.72 & 4 & 4 \\
\hline 99 & $\mathrm{Csp} \mathrm{sp}^{2}$ & $\mathrm{~N} 2=\mathrm{N}(+)$ & 65.95 & 6 & 5 \\
\hline 100 & $\mathrm{Csp}^{2}$ & $\mathrm{~N} 2=\mathrm{O}$ & 59.57 & 76 & 70 \\
\hline 101 & $\mathrm{Csp} \mathrm{sp}^{2}$ & $\mathrm{~N} 2=\mathrm{S}$ & 66.62 & 29 & 29 \\
\hline 102 & $\mathrm{C} \mathrm{sp}^{2}$ & $\mathrm{~N}=\mathrm{NS}$ & 51.62 & 22 & 22 \\
\hline 103 & $\mathrm{Csp} \mathrm{sp}^{2}$ & $\mathrm{NO}=\mathrm{O}$ & 52.79 & 8 & 8 \\
\hline 104 & $\mathrm{Csp} \mathrm{sp}^{2}$ & $=\mathrm{NO} 2$ & 61.12 & 1 & 1 \\
\hline 105 & $\mathrm{C} \mathrm{sp}^{2}$ & $\mathrm{~N}=\mathrm{OS}$ & 48.27 & 1 & 1 \\
\hline 106 & $\mathrm{Csp} \mathrm{sp}^{2}$ & $\mathrm{NO}=\mathrm{S}$ & 58.04 & 11 & 11 \\
\hline 107 & $\mathrm{Csp} \mathrm{sp}^{2}$ & $=\mathrm{NOS}$ & 52.75 & 1 & 1 \\
\hline 108 & $\mathrm{Csp}^{2}$ & $\mathrm{NS}=\mathrm{S}$ & 60.83 & 5 & 3 \\
\hline 109 & $\mathrm{Csp} \mathrm{sp}^{2}$ & $=\mathrm{NS} 2$ & 64.37 & 1 & 1 \\
\hline 110 & $\mathrm{Csp}^{2}$ & $\mathrm{O} 2=\mathrm{O}$ & 41.40 & 7 & 7 \\
\hline 111 & $\mathrm{Csp}^{2}$ & $=\mathrm{OS} 2$ & 41.22 & 2 & 2 \\
\hline 112 & $\mathrm{Csp} \mathrm{sp}^{2}$ & $\mathrm{OS}=\mathrm{S}$ & 73.06 & 1 & 1 \\
\hline 113 & $\mathrm{Csp}^{2}$ & $\mathrm{~S} 2=\mathrm{S}$ & 49.39 & 5 & 5 \\
\hline 114 & $\mathrm{C}$ aromatic & $\mathrm{H}: \mathrm{C} 2$ & 5.36 & 7115 & 1269 \\
\hline 115 & $\mathrm{C}$ aromatic & $\mathrm{H}: \mathrm{C}: \mathrm{N}$ & 18.20 & 150 & 96 \\
\hline 116 & C aromatic & $\mathrm{H}: \mathrm{C}: \mathrm{N}(+)$ & 28.26 & 48 & 28 \\
\hline 117 & C aromatic & $\mathrm{H}: \mathrm{N} 2$ & 23.27 & 7 & 5 \\
\hline 118 & $\mathrm{C}$ aromatic & $\mathrm{B}: \mathrm{C} 2$ & -25.04 & 3 & 1 \\
\hline 119 & $\mathrm{C}$ aromatic & :C3 & 5.51 & 454 & 155 \\
\hline 120 & C aromatic & $\mathrm{C}: \mathrm{C} 2$ & 3.12 & 1684 & 835 \\
\hline 121 & C aromatic & $\mathrm{C}: \mathrm{C}: \mathrm{N}$ & 11.10 & 80 & 48 \\
\hline 122 & $\mathrm{C}$ aromatic & $\mathrm{C}: \mathrm{C}: \mathrm{N}(+)$ & 16.04 & 33 & 21 \\
\hline 123 & C aromatic & $: \mathrm{C} 2 \mathrm{~N}$ & 22.21 & 354 & 258 \\
\hline 124 & C aromatic & $: \mathrm{C} 2 \mathrm{~N}(+)$ & 28.67 & 169 & 134 \\
\hline 125 & C aromatic & $: \mathrm{C} 2 \mathrm{~N}$ & 17.03 & 79 & 61 \\
\hline 126 & $\mathrm{C}$ aromatic & $: \mathrm{C} 2: \mathrm{N}(+)$ & 18.05 & 35 & 20 \\
\hline 127 & C aromatic & $: \mathrm{C} 2 \mathrm{O}$ & 20.46 & 617 & 387 \\
\hline 128 & $\mathrm{C}$ aromatic & $: \mathrm{C} 2 \mathrm{P}$ & -1.63 & 12 & 4 \\
\hline 129 & C aromatic & :C2S & 16.31 & 80 & 64 \\
\hline 130 & $\mathrm{C}$ aromatic & $: \mathrm{C} 2 \mathrm{~F}$ & 4.45 & 77 & 42 \\
\hline 131 & C aromatic & $: \mathrm{C} 2 \mathrm{Cl}$ & 12.48 & 424 & 166 \\
\hline 132 & $\mathrm{C}$ aromatic & :C2Br & 14.66 & 63 & 43 \\
\hline 133 & C aromatic & :C2J & 20.68 & 31 & 27 \\
\hline 134 & C aromatic & :C2Si & 4.80 & 10 & 2 \\
\hline 135 & $\mathrm{C}$ aromatic & $\mathrm{C}: \mathrm{N} 2$ & 28.80 & 4 & 2 \\
\hline 136 & C aromatic & $: \mathrm{CN}: \mathrm{N}$ & 29.72 & 11 & 9 \\
\hline 137 & $\mathrm{C}$ aromatic & :CN:N(+) & 33.74 & 3 & 2 \\
\hline 138 & C aromatic & :C:NO & 41.44 & 13 & 12 \\
\hline 139 & $\mathrm{C}$ aromatic & :C:NO(+) & 33.50 & 5 & 5 \\
\hline 140 & C aromatic & :C:NCl & 21.70 & 18 & 13 \\
\hline 141 & $\mathrm{C}$ aromatic & :C:NBr & 31.31 & 3 & 2 \\
\hline 142 & C aromatic & N:N2 & 43.11 & 13 & 8 \\
\hline 143 & $\mathrm{C}$ aromatic & :N2O & 39.92 & 3 & 1 \\
\hline 144 & $\mathrm{C}$ aromatic & :N2S & 36.08 & 3 & 3 \\
\hline
\end{tabular}


Table 2. Cont.

\begin{tabular}{|c|c|c|c|c|c|}
\hline Entry & Atom Type & Neighbours & Contribution & Occurrences & Molecules \\
\hline 145 & C aromatic & :N2Cl & 35.90 & 3 & 3 \\
\hline 146 & C sp & $=\mathrm{C} 2$ & 6.39 & 3 & 2 \\
\hline 147 & $\mathrm{Csp}$ & $\mathrm{C \# C}$ & 3.24 & 14 & 7 \\
\hline 148 & C sp & $\mathrm{C \# N}$ & 16.49 & 96 & 67 \\
\hline 149 & $\mathrm{Csp}$ & $\mathrm{C \# N(+)}$ & 11.33 & 4 & 3 \\
\hline 150 & C sp & \#CS & 28.03 & 2 & 2 \\
\hline 151 & $\mathrm{C} \mathrm{sp}$ & $\mathrm{N \# N}$ & 47.80 & 1 & 1 \\
\hline 152 & C sp & \#NP & 12.53 & 3 & 1 \\
\hline 153 & $\mathrm{Nsp}^{3}$ & $\mathrm{H} 2 \mathrm{C}$ & 5.03 & 23 & 12 \\
\hline 154 & $\mathrm{~N} \mathrm{sp}^{3}$ & $\mathrm{H} 2 \mathrm{C}(\mathrm{pi})$ & 6.38 & 223 & 199 \\
\hline 155 & $\mathrm{~N} \mathrm{sp}^{3}$ & $\mathrm{H} 2 \mathrm{~N}$ & 17.97 & 10 & 8 \\
\hline 156 & $\mathrm{~N} \mathrm{sp}^{3}$ & $\mathrm{H} 2 \mathrm{~S}$ & 41.98 & 1 & 1 \\
\hline 157 & $\mathrm{~N} \mathrm{sp}^{3}$ & $\mathrm{HC} 2$ & -23.83 & 14 & 13 \\
\hline 158 & $\mathrm{~N} \mathrm{sp}^{3}$ & HC2(pi) & -13.51 & 72 & 55 \\
\hline 159 & $\mathrm{~N} \mathrm{sp}^{3}$ & $\mathrm{HC} 2(2 \mathrm{pi})$ & -20.10 & 200 & 165 \\
\hline 160 & $\mathrm{~N} \mathrm{sp}^{3}$ & $\mathrm{HCN}$ & -0.15 & 2 & 1 \\
\hline 161 & $\mathrm{~N} \mathrm{sp}^{3}$ & $\mathrm{HCN}(\mathrm{pi})$ & 6.71 & 14 & 9 \\
\hline 162 & $\mathrm{~N} \mathrm{sp}^{3}$ & $\mathrm{HCN}(2 \mathrm{pi})$ & -6.84 & 25 & 25 \\
\hline 163 & $\mathrm{~N} \mathrm{sp}^{3}$ & $\mathrm{HCS}(\mathrm{pi})$ & -15.10 & 20 & 20 \\
\hline 164 & $\mathrm{~N} \mathrm{sp}^{3}$ & $\mathrm{C} 3$ & -51.07 & 16 & 11 \\
\hline 165 & $\mathrm{~N} \mathrm{sp}^{3}$ & C3(pi) & -53.90 & 59 & 49 \\
\hline 166 & $\mathrm{~N} \mathrm{sp}^{3}$ & C3(2pi) & -60.80 & 72 & 54 \\
\hline 167 & $\mathrm{~N} \mathrm{sp}^{3}$ & C3(3pi) & -61.26 & 18 & 14 \\
\hline 168 & $\mathrm{~N} \mathrm{sp}^{3}$ & $\mathrm{C} 2 \mathrm{~N}(\mathrm{pi})$ & -7.05 & 6 & 3 \\
\hline 169 & $\mathrm{~N} \mathrm{sp}^{3}$ & $\mathrm{C} 2 \mathrm{~N}(+)(\mathrm{pi})$ & -5.52 & 24 & 9 \\
\hline 170 & $\mathrm{~N} \mathrm{sp}^{3}$ & $\mathrm{C} 2 \mathrm{~N}(2 \mathrm{pi})$ & -36.36 & 4 & 4 \\
\hline 171 & $\mathrm{~N} \mathrm{sp}^{3}$ & $\mathrm{C} 2 \mathrm{~N}(+)(2 \mathrm{pi})$ & -20.13 & 1 & 1 \\
\hline 172 & $\mathrm{~N} \mathrm{sp}^{3}$ & C2N(3pi) & -54.74 & 3 & 3 \\
\hline 173 & $\mathrm{~N} \mathrm{sp}^{3}$ & $\mathrm{C} 2 \mathrm{~S}$ & -49.13 & 4 & 2 \\
\hline 174 & $\mathrm{~N} \mathrm{sp}^{3}$ & $\mathrm{C} 2 \mathrm{~F}(2 \mathrm{pi})$ & -64.78 & 1 & 1 \\
\hline 175 & $\mathrm{~N} \mathrm{sp}^{3}$ & CN2(pi) & 30.74 & 4 & 3 \\
\hline 176 & $\mathrm{~N} \mathrm{sp}^{3}$ & CN2(2pi) & -49.40 & 3 & 3 \\
\hline 177 & $\mathrm{~N} \mathrm{sp}^{3}$ & $\mathrm{CN} 2(+)(2 \mathrm{pi})$ & 3.72 & 1 & 1 \\
\hline 178 & $\mathrm{~N} \mathrm{sp}^{3}$ & CNF(2pi) & -34.74 & 5 & 4 \\
\hline 179 & $\mathrm{~N} \mathrm{sp}^{2}$ & $\mathrm{C}=\mathrm{C}$ & -32.77 & 79 & 74 \\
\hline 180 & $\mathrm{~N} \mathrm{sp}{ }^{2}$ & $\mathrm{C}=\mathrm{N}$ & -4.54 & 13 & 9 \\
\hline 181 & $\mathrm{Nsp}^{2}$ & $\mathrm{C}=\mathrm{N}(+)$ & -15.43 & 5 & 5 \\
\hline 182 & $\mathrm{~N} \mathrm{sp}^{2}$ & $=\mathrm{CN}$ & -4.63 & 38 & 36 \\
\hline 183 & $\mathrm{~N} \mathrm{sp}^{2}$ & $=\mathrm{CN}(+)$ & 36.68 & 1 & 1 \\
\hline 184 & $\mathrm{Nsp}^{2}$ & $\mathrm{C}=\mathrm{O}$ & -12.04 & 9 & 9 \\
\hline 185 & $\mathrm{~N} \mathrm{sp}^{2}$ & $\mathrm{C}=\mathrm{P}$ & -49.18 & 1 & 1 \\
\hline 186 & $\mathrm{~N} \mathrm{sp}^{2}$ & $=\mathrm{CO}$ & -16.24 & 18 & 13 \\
\hline 187 & $\mathrm{~N} \mathrm{sp}^{2}$ & $=\mathrm{CS}$ & -26.78 & 10 & 8 \\
\hline 188 & $\mathrm{~N} \mathrm{sp}^{2}$ & $\mathrm{~N}=\mathrm{N}$ & 12.19 & 21 & 13 \\
\hline 189 & $\mathrm{~N} \mathrm{sp}^{2}$ & $\mathrm{~N}=\mathrm{O}$ & 0.00 & 10 & 6 \\
\hline 190 & $\mathrm{Nsp}^{2}$ & $=\mathrm{NO}$ & -6.67 & 2 & 1 \\
\hline 191 & $\mathrm{~N}$ aromatic & :C2 & -14.01 & 208 & 145 \\
\hline 192 & $\mathrm{~N}$ aromatic & $: \mathrm{C}: \mathrm{N}$ & -4.98 & 4 & 2 \\
\hline 193 & $\mathrm{~N}(+) \mathrm{sp}^{3}$ & Н3С & 2.77 & 13 & 13 \\
\hline 194 & $\mathrm{~N}(+) \mathrm{sp}^{3}$ & $\mathrm{H} 2 \mathrm{C} 2$ & -82.36 & 3 & 3 \\
\hline 195 & $\mathrm{~N}(+) \mathrm{sp}^{2}$ & $\mathrm{C}=\mathrm{CO}(-)$ & -68.61 & 7 & 7 \\
\hline 196 & $\mathrm{~N}(+) \mathrm{sp}^{2}$ & $\mathrm{C}=\mathrm{NO}$ & -26.37 & 10 & 5 \\
\hline 197 & $\mathrm{~N}(+) \mathrm{sp}^{2}$ & $\mathrm{C}=\mathrm{NO}(-)$ & -11.30 & 3 & 3 \\
\hline 198 & $\mathrm{~N}(+) \mathrm{sp}^{2}$ & $\mathrm{CO}=\mathrm{O}(-)$ & -4.38 & 270 & 163 \\
\hline 199 & $\mathrm{~N}(+) \mathrm{sp}^{2}$ & $=\mathrm{CO} 2(-)$ & 2.17 & 5 & 5 \\
\hline 200 & $\mathrm{~N}(+) \mathrm{sp}^{2}$ & $\mathrm{NO}=\mathrm{O}(-)$ & 0.15 & 28 & 12 \\
\hline
\end{tabular}


Table 2. Cont

\begin{tabular}{|c|c|c|c|c|c|}
\hline Entry & Atom Type & Neighbours & Contribution & Occurrences & Molecules \\
\hline 201 & $\mathrm{~N}(+) \mathrm{sp}^{2}$ & $\mathrm{O} 2=\mathrm{O}(-)$ & 6.00 & 14 & 6 \\
\hline 202 & $\mathrm{~N}(+)$ aromatic & $\mathrm{H}: \mathrm{C} 2$ & -46.79 & 6 & 6 \\
\hline 203 & $\mathrm{~N}(+)$ aromatic & $: \mathrm{C} 2 \mathrm{O}(-)$ & -7.10 & 56 & 40 \\
\hline 204 & $\mathrm{~N}(+) \mathrm{sp}$ & C\#C(-) & -14.36 & 3 & 3 \\
\hline 205 & $\mathrm{~N}(+) \mathrm{sp}$ & \#CO(-) & 0.00 & 4 & 3 \\
\hline 206 & $\mathrm{~N}(+) \mathrm{sp}$ & $=\mathrm{N} 2(-)$ & 19.14 & 2 & 2 \\
\hline 207 & O & $\mathrm{HC}$ & 4.49 & 143 & 92 \\
\hline 208 & $\mathrm{O}$ & $\mathrm{HC}(\mathrm{pi})$ & 8.19 & 560 & 470 \\
\hline 209 & $\mathrm{O}$ & $\mathrm{HN}(\mathrm{pi})$ & 2.28 & 4 & 3 \\
\hline 210 & $\mathrm{O}$ & $\mathrm{HO}$ & 29.95 & 4 & 4 \\
\hline 211 & $\mathrm{O}$ & $\mathrm{C} 2$ & -39.23 & 94 & 37 \\
\hline 212 & $\mathrm{O}$ & C2(pi) & -31.33 & 292 & 201 \\
\hline 213 & $\mathrm{O}$ & $\mathrm{C} 2(2 \mathrm{pi})$ & -24.06 & 147 & 121 \\
\hline 214 & $\mathrm{O}$ & $\mathrm{CN}(\mathrm{pi})$ & 0.00 & 2 & 1 \\
\hline 215 & $\mathrm{O}$ & $\mathrm{CN}(+)(\mathrm{pi})$ & 0.00 & 14 & 6 \\
\hline 216 & $\mathrm{O}$ & $\mathrm{CN}(2 \mathrm{pi})$ & 4.91 & 1 & 1 \\
\hline 217 & $\mathrm{O}$ & $\mathrm{CO}(\mathrm{pi})$ & -27.16 & 8 & 6 \\
\hline 218 & $\mathrm{O}$ & $\mathrm{CP}(\mathrm{pi})$ & -16.12 & 3 & 1 \\
\hline 219 & $\mathrm{O}$ & N2(2pi) & 5.87 & 4 & 4 \\
\hline 220 & $\mathrm{O}$ & $\mathrm{N} 2(+)(2 \mathrm{pi})$ & 6.27 & 5 & 5 \\
\hline 221 & P3 & $\mathrm{C} 3$ & 16.70 & 2 & 2 \\
\hline 222 & P3 & S3 & -66.68 & 1 & 1 \\
\hline 223 & $\mathrm{P} 4$ & $\mathrm{C} 3=\mathrm{N}$ & 0.00 & 1 & 1 \\
\hline 224 & $\mathrm{P} 4$ & $\mathrm{C} 3=\mathrm{O}$ & -30.50 & 1 & 1 \\
\hline 225 & $\mathrm{P} 4$ & $\mathrm{C} 3=\mathrm{S}$ & 46.30 & 1 & 1 \\
\hline 226 & $\mathrm{P} 4$ & $\mathrm{O} 3=\mathrm{O}$ & 0.00 & 1 & 1 \\
\hline 227 & $\mathrm{~S} 2$ & $\mathrm{HC}$ & -2.58 & 1 & 1 \\
\hline 228 & $\mathrm{~S} 2$ & $\mathrm{HC}(\mathrm{pi})$ & 18.47 & 2 & 2 \\
\hline 229 & $\mathrm{~S} 2$ & $\mathrm{C} 2$ & -22.69 & 19 & 12 \\
\hline 230 & $\mathrm{~S} 2$ & C2(pi) & -15.86 & 34 & 29 \\
\hline 231 & $\mathrm{~S} 2$ & $\mathrm{C} 2(2 \mathrm{pi})$ & -7.94 & 59 & 49 \\
\hline 232 & $\mathrm{~S} 2$ & $\mathrm{CN}(\mathrm{pi})$ & 25.96 & 1 & 1 \\
\hline 233 & S2 & CN(2pi) & -6.82 & 6 & 6 \\
\hline 234 & $\mathrm{~S} 2$ & $\mathrm{CS}(\mathrm{pi})$ & -6.16 & 8 & 4 \\
\hline 235 & $\mathrm{~S} 2$ & $\mathrm{CP}(\mathrm{pi})$ & 0.00 & 3 & 1 \\
\hline 236 & $\mathrm{~S} 2$ & N2 & -2.00 & 1 & 1 \\
\hline 237 & $\mathrm{~S} 2$ & N2(2pi) & 21.36 & 2 & 2 \\
\hline 238 & $\mathrm{~S} 2$ & NS & 1.00 & 2 & 1 \\
\hline 239 & $\mathrm{~S} 4$ & $\mathrm{C} 2=\mathrm{O}$ & -5.89 & 2 & 2 \\
\hline 240 & $\mathrm{~S} 4$ & $\mathrm{C} 2=\mathrm{O} 2$ & -4.26 & 27 & 27 \\
\hline 241 & $\mathrm{~S} 4$ & $\mathrm{CN}=\mathrm{O} 2$ & 9.20 & 20 & 20 \\
\hline 242 & $\mathrm{Si}$ & $\mathrm{C} 4$ & 2.02 & 1 & 1 \\
\hline 243 & $\mathrm{Si}$ & $\mathrm{C} 3 \mathrm{Si}$ & -0.67 & 2 & 1 \\
\hline 244 & $\mathrm{H}$ & H Acceptor & -8.63 & 107 & 89 \\
\hline 245 & Alkane & No. of $C$ atoms & -0.53 & 849 & 59 \\
\hline 246 & Unsaturated HC & No. of $\mathrm{C}$ atoms & -0.10 & 2679 & 148 \\
\hline A & Based on & Valid groups & 154 & & 1960 \\
\hline B & Goodness of fit & $R^{2}$ & 0.8887 & & 1866 \\
\hline $\mathrm{C}$ & Deviation & Average & 7.81 & & 1866 \\
\hline $\mathrm{D}$ & Deviation & Standard & 10.33 & & 1866 \\
\hline $\mathrm{E}$ & $\mathrm{K}$-fold cv & $\mathrm{K}$ & 10 & & 1791 \\
\hline $\mathrm{F}$ & Goodness of fit & $Q^{2}$ & 0.8657 & & 1791 \\
\hline G & Deviation & Average (cv) & 8.56 & & 1791 \\
\hline $\mathrm{H}$ & Deviation & Standard (cv) & 11.39 & & 1791 \\
\hline
\end{tabular}

The correlation coefficients $R^{2}$ and $Q^{2}$ (rows B and $\mathrm{F}$ at the bottom of Table 2) exhibit a higher scatter of the experimental data in comparison with the heat-of-vaporization data. The increased 
uncertainty might be partly ascribed to the fact that in many cases molecules form several crystal structures at different temperatures, having different enthalpies of fusion, and that, therefore, the starting point of the measurements is not clearly defined. In other cases the molecules may not be completely crystalline due to impurities. Another reason may be that while many of the compounds in the enthalpy of the vaporization dataset of the previous chapter are liquid at ambient room temperature and the vaporization measurements have been performed at temperatures not too far removed from $298.15 \mathrm{~K}$, requiring only a small correction back to this reference temperature, the enthalpy of sublimation measurements, on the other hand, are often carried out at higher temperatures where the compounds are more volatile. In these cases, the uncertainty in the correction term needed to extrapolate the experimental value back to the reference temperature is higher and increases with the difference between the experimental and the reference temperature. The consequences of these uncertainties are reflected in the spread of experimental data originating from different authors for the same compounds: for example, for the enthalpy of sublimation of anthracene, there are seven values given in the 2010 Acree and Chickos compilation [8] that range from 88.3 to $93.3 \mathrm{~kJ} / \mathrm{mol}$, and for coumarin there are two values for the same property that range from 83.1 to $95.4 \mathrm{~kJ} / \mathrm{mol}$.

Figure 3 demonstrates the larger scatter of the data about the regression line, leading to a cross-validated standard deviation of 11.39 (see row $\mathrm{H}$ in Table 2), i.e., 2.5 times larger than for the heat of vaporization. Figure 4 visualizes the error distribution, showing that, according to an analysis, $74 \%$ of the molecules' predicted values differ by less one cv-standard deviation and only $5.6 \%$ by more than twice that amount. Only 16 compunds had to be declared as outliers because their experimental value exceeded four times the cv-standard deviation. One compound, norcamphor, had to be excluded from calculation because its experimental enthalpy of sublimation was lower than its experimental enthalpy of vaporization, an obviously impossible finding.

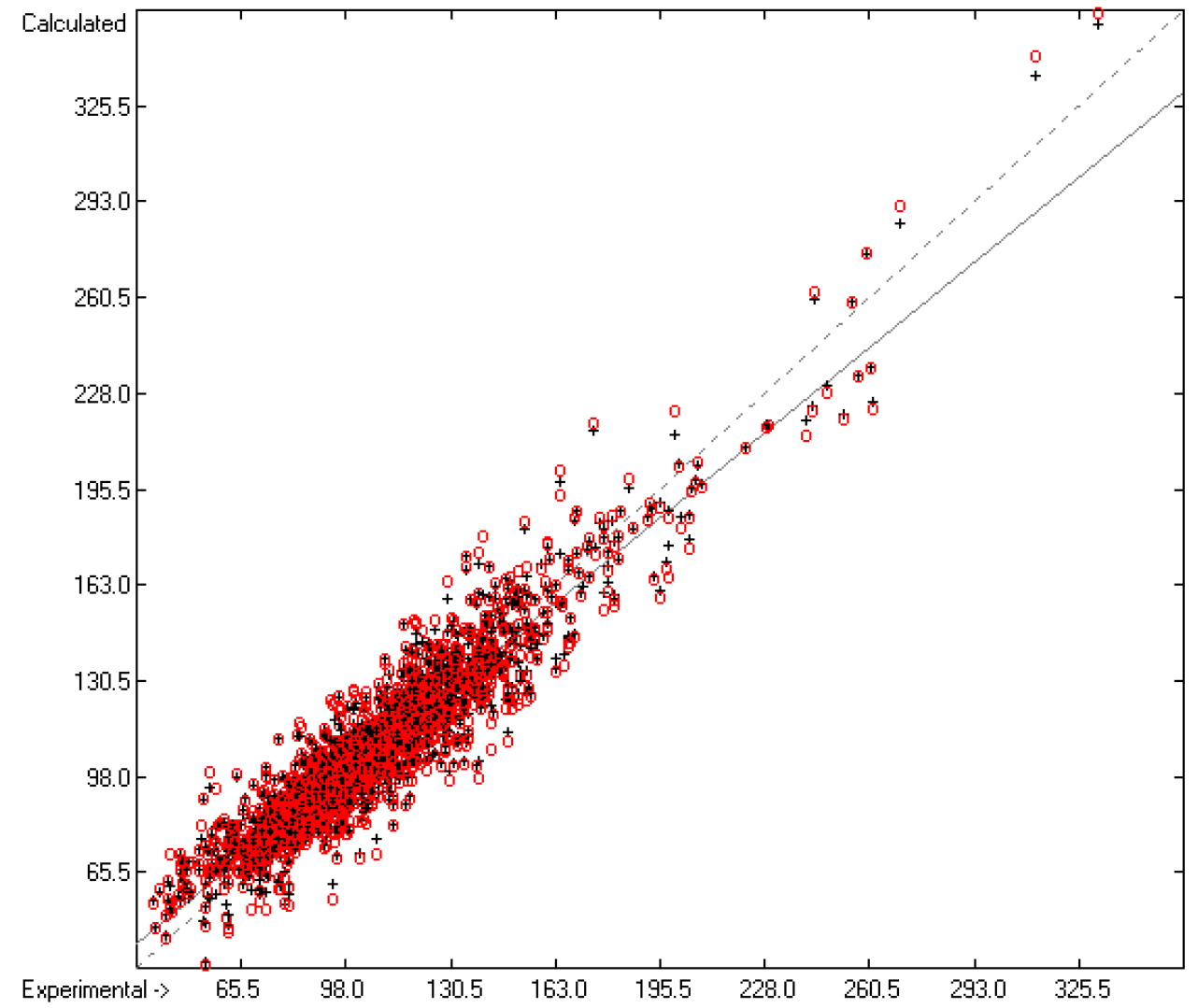

Figure 3. Correlation Diagram of the Enthalpy-of-Sublimation Data $\left(N=1866 ; R^{2}=0.8887 ; Q^{2}=0.8657\right.$; regression line: intercept $=12.0233$, slope $=0.8884$ ). 


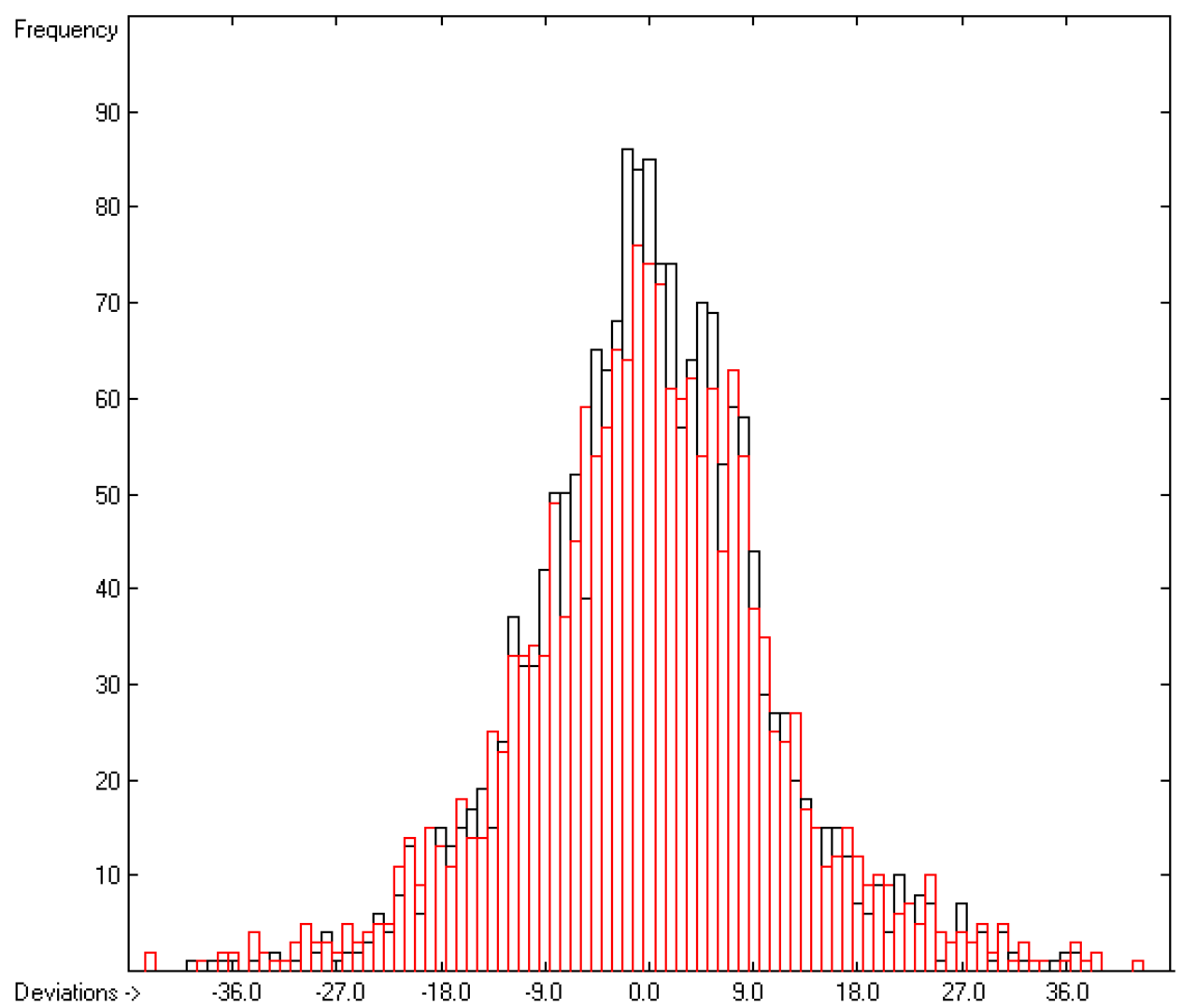

Figure 4. Histogram of the Enthalpy-of-Sublimation Data $(S=11.39 \mathrm{~kJ} / \mathrm{mol}$; Exp. values range: $38.7-331.88 \mathrm{~kJ} / \mathrm{mol})$.

The lower number of "valid" atom groups of 154, as shown in row A of Table 2, led to the slightly reduced amount of $75.9 \%$ of the molecules in the representative database for which the heat of sublimation was calculable.

\subsection{Enthalpy of Fusion}

It seems obvious to try to apply the atom-group additivity method as described in the preceding chapters for the prediction of the enthalpy of fusion, all the more as several authors $[21,60]$ have already used this principle very successfully. However, since the presented predictions of the enthalpies of sublimation and vaporization rest exclusively on experimental values at $298 \mathrm{~K}$, it is legitimate to refer to the simple Equation (2) which defines a molecule's enthalpy of fusion at standard conditions as the difference between its enthalpy of sublimation and its enthalpy of vaporization:

$$
\Delta H^{\circ} \text { fus }(298 \mathrm{~K}) \text { calc. }=\Delta H_{\text {sub }}^{\circ}(298 \mathrm{~K}) \text { calc. }-\Delta H^{\circ}{ }_{\text {vap }}(298 \mathrm{~K}) \text { calc. }
$$

Accordingly, the standard deviation of the thus evaluated enthalpy of fusion can be calculated by means of the error-propagation equation for the sum of two cross-validation standard errors $Q^{2}\left(\Delta H^{\circ}{ }_{\text {sub }}\right)(=11.39 \mathrm{~kJ} / \mathrm{mol})$ and $Q^{2}\left(\Delta H^{\circ}{ }_{\text {vap }}\right)(=4.56 \mathrm{~kJ} / \mathrm{mol})$, resulting in a standard deviation $\sigma$ for the calculated enthalpy of fusion of $12.27 \mathrm{~kJ} / \mathrm{mol}$. Evidently, this deviation is largely dominated by the uncertainty of the experimental heats of sublimation and, thus would gain the most upon the provision of more accurate sublimation data.

How well do the predictions of Equation (2) compare to experimental heat-of-fusion data? In order to answer this question more than 1200 experimental values have been inserted into the database, taken from Acree's compendium publication [8], complemented by recent values for crown ethers [29], 
fluorinated alcohols [33], adamantanes [53], 2-chloro-3-(trifluoromethyl)pyridine [61], cyanatophenyl derivatives [62], diphenylamines [63], fatty acids [64], pyridinecarbothioamides [65], isoniazid [66] and phenylthiazole-thione [67]. Figure 5 shows a comparison of the experimental with the predicted values, independently calculated by means of Equation (2). After removal of the worst 28 outliers the correlation coefficient $R^{2}$ for the remaining 990 samples (for which both the experimental and predicted values were available) was calculated to 0.60 . This rather low value is at least in part explicable by findings outlined in several papers revealing that for certain compounds experimental values originating from different authors often scatter over a large range. For instance, Eckert et al. [64] graphically demonstrated for various fatty acids that the value of their enthalpy of fusion varied drastically over a period of up to 80 years of repeated examination. Some examples: the enthalpy of fusion for palmitic acid randomly varied over the years between ca. 41 and $60 \mathrm{~kJ} / \mathrm{mol}$, and for stearic acid the range, varying between ca. 45 and $74 \mathrm{~kJ} / \mathrm{mol}$, was even wider. Analogous observations were made by Leitner and Jurik [68], who discovered similar discrepancies by different authors also for small molecules, exemplified by paracetamol and aspirin, for which the published values varied between 26 and 34.1, and between 29.89 and $32.92 \mathrm{~kJ} / \mathrm{mol}$, respectively. Figure 5, also demonstrating that the overwhelming number of experimental data is concentrated in the narrow range of below $40 \mathrm{~kJ} / \mathrm{mol}$, provides another explanation for the difficulty to enable exact predictions. The related histogram in Figure 6 nevertheless proves a satisfyingly even distribution of the deviations about the regression line drawn in Figure 5. Thanks to the broad applicability of the "valid" number of atom-group parameters for both the heat of sublimation as well as the heat of vaporization, Equation (2) enabled the estimation of the heat of fusion of $68 \%$ of the database's molecules.

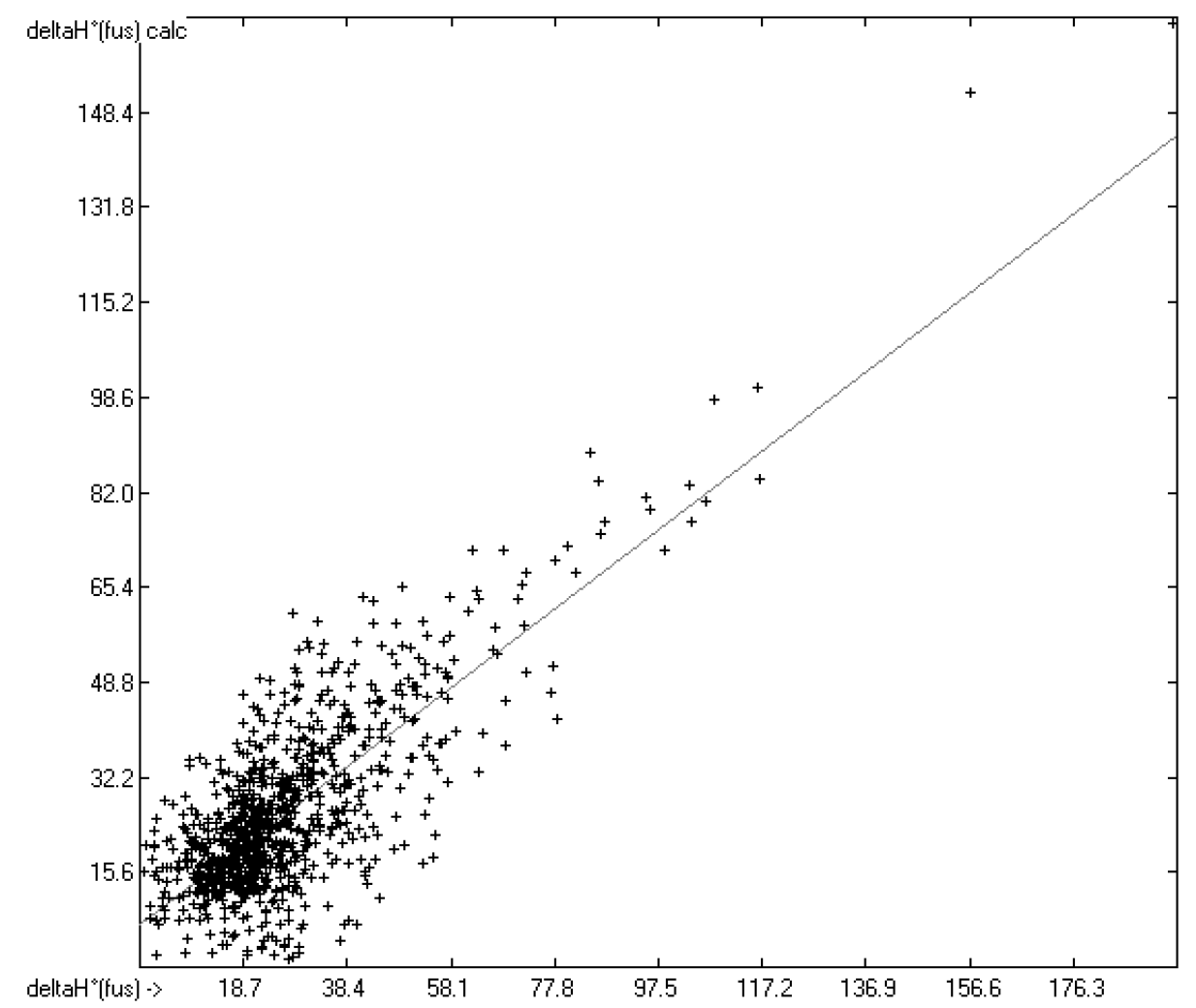

Figure 5. Correlation Diagram of the Enthalpy-of-Fusion Data $\left(N=990 ; R^{2}=0.6066\right.$; calculated values evaluated by means of Equation (2)). 


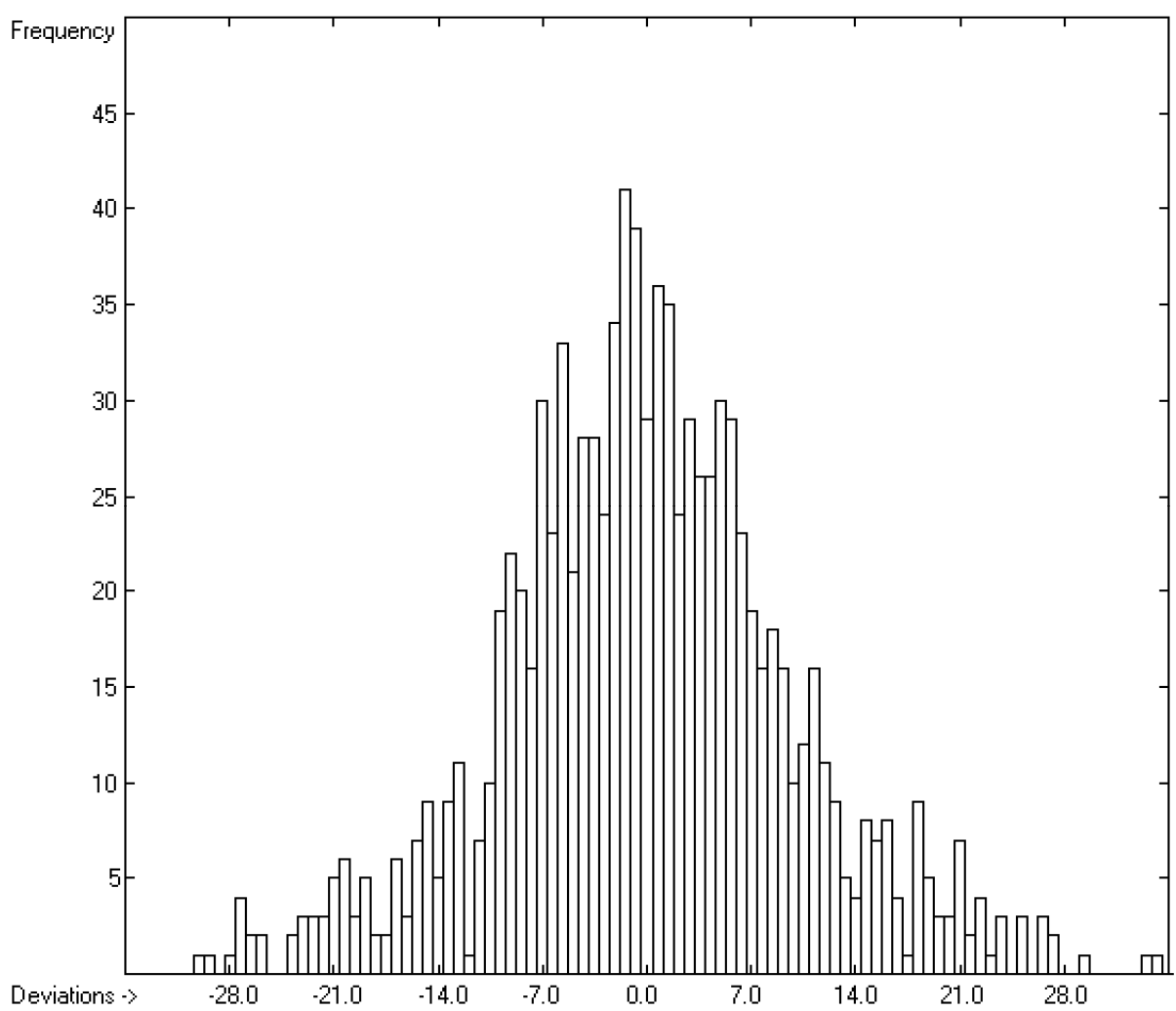

Figure 6. Histogram of the Enthalpy-of-Fusion Data $(S=9.78 \mathrm{~kJ} / \mathrm{mol}$; Exp. values range: $0.30-164 \mathrm{~kJ} / \mathrm{mol})$.

\subsection{Enthalpy of Solvation}

Literature referencing experimental enthalpy-of-solvation data is relatively scarce. The most yielding source was found in Mintz et al.'s [69] paper on the application of the Abraham model mentioned earlier on gaseous solutes dissolved in water and 1-octanol. Further studies were made on $\mathrm{N}$-methylimidazole [70], urea and its derivatives [71-73], thiourea and its derivatives [74], carboxamides and their $\mathrm{N}$-substituted derivatives [75-78], and uracil and its alkyl-, amino-, nitro- and halosubstituted derivatives [79-82]. Of the accordingly limited number of 465 compounds having experimental enthalpy-of-solvation values for water as solvent in their datalist, 436 have been entered into the calculation of the atom-group parameters, resulting in 61 valid groups allowing the evaluation of the cross-validated prediction of the solvation enthalpy of 373 compounds with a cv-goodness of fit of 0.9546 and a corresponding standard deviation $Q^{2}$ of $4.34 \mathrm{~kJ} / \mathrm{mol}$ as is shown in aggregated manner in Table 3.

Table 3. Atom Groups and their Contributions (in $\mathrm{kJ} / \mathrm{mol}$ ) for Heat-of-Solvation Calculations.

\begin{tabular}{cccccc}
\hline Entry & Atom Type & Neighbours & Contribution & Occurrences & Molecules \\
\hline 1 & Const & & -13.33 & 436 & 436 \\
2 & $\mathrm{C} \mathrm{sp}{ }^{3}$ & $\mathrm{H} 3 \mathrm{C}$ & -4.44 & 483 & 265 \\
3 & $\mathrm{C} \mathrm{sp}$ & $\mathrm{H} 3 \mathrm{~N}$ & -31.51 & 47 & 28 \\
4 & $\mathrm{C} \mathrm{sp}$ & $\mathrm{H} 3 \mathrm{~N}(+)$ & -31.22 & 1 & 1 \\
5 & $\mathrm{C} \mathrm{sp}$ & $\mathrm{H} 3 \mathrm{O}$ & -15.38 & 34 & 29 \\
6 & $\mathrm{C} \mathrm{sp}$ & $\mathrm{H} 3 \mathrm{~S}$ & -12.79 & 7 & 4 \\
7 & $\mathrm{H} \mathrm{Cs}^{3}$ & $\mathrm{H} 2 \mathrm{CN}$ & -3.86 & 506 & 36 \\
8 & $\mathrm{C} \mathrm{sp}$ & $\mathrm{H} 2 \mathrm{CN}(+)$ & -31.29 & 55 & 2 \\
9 & $\mathrm{C} \mathrm{sp}$ & $\mathrm{H} 2 \mathrm{CO}$ & -15.60 & 178 & 90 \\
10 & $\mathrm{C} \mathrm{sp}$ & & & & 26 \\
\hline
\end{tabular}


Table 3. Cont.

\begin{tabular}{|c|c|c|c|c|c|}
\hline Entry & Atom Type & Neighbours & Contribution & Occurrences & Molecules \\
\hline 11 & $\mathrm{Csp}^{3}$ & $\mathrm{H} 2 \mathrm{CS}$ & -12.03 & 9 & 6 \\
\hline 12 & $\mathrm{Csp}$ & $\mathrm{H} 2 \mathrm{CF}$ & -6.02 & 1 & 1 \\
\hline 13 & $\mathrm{Csp}$ & $\mathrm{H} 2 \mathrm{CCl}$ & -8.52 & 15 & 11 \\
\hline 14 & $\mathrm{Csp}$ & $\mathrm{H} 2 \mathrm{CBr}$ & -11.73 & 1 & 1 \\
\hline 15 & $\mathrm{Csp}$ & $\mathrm{H} 2 \mathrm{CJ}$ & -13.80 & 2 & 2 \\
\hline 16 & $\mathrm{Csp}$ & $\mathrm{H} 2 \mathrm{O} 2$ & -14.86 & 1 & 1 \\
\hline 17 & $\mathrm{Csp}$ & $\mathrm{HC} 3$ & -2.51 & 45 & 35 \\
\hline 18 & $\mathrm{Csp}$ & $\mathrm{HC} 2 \mathrm{~N}$ & -29.99 & 6 & 5 \\
\hline 19 & $\mathrm{Csp}$ & $\mathrm{HC} 2 \mathrm{~N}(+)$ & -20.74 & 1 & 1 \\
\hline 20 & $\mathrm{Csp}$ & $\mathrm{HC} 2 \mathrm{O}$ & -14.95 & 32 & 29 \\
\hline 21 & $\mathrm{Csp}$ & $\mathrm{HC} 2 \mathrm{~F}$ & -5.77 & 1 & 1 \\
\hline 22 & $\mathrm{Csp}$ & $\mathrm{HC} 2 \mathrm{Cl}$ & -8.53 & 1 & 1 \\
\hline 23 & $\mathrm{C} \mathrm{sp}^{3}$ & HC2J & -14.39 & 1 & 1 \\
\hline 24 & $\mathrm{C} \mathrm{sp}^{3}$ & HCF2 & -5.07 & 3 & 3 \\
\hline 25 & $\mathrm{Csp}$ & $\mathrm{HCCl} 2$ & -11.02 & 5 & 4 \\
\hline 26 & $\mathrm{Csp}$ & C4 & 0.43 & 10 & 10 \\
\hline 27 & $\mathrm{Csp}$ & $\mathrm{C} 3 \mathrm{~N}$ & -24.37 & 3 & 3 \\
\hline 28 & $\mathrm{Csp}$ & $\mathrm{C} 3 \mathrm{O}$ & -16.23 & 6 & 6 \\
\hline 29 & $\mathrm{Csp}$ & $\mathrm{C} 3 \mathrm{Cl}$ & -1.29 & 1 & 1 \\
\hline 30 & $\mathrm{Csp}$ & $\mathrm{C} 3 \mathrm{Br}$ & 1.24 & 1 & 1 \\
\hline 31 & $\mathrm{Csp} \mathrm{sp}^{3}$ & C3J & -7.51 & 1 & 1 \\
\hline 32 & $\mathrm{Csp}$ & $\mathrm{C} 2 \mathrm{~F} 2$ & -5.12 & 2 & 2 \\
\hline 33 & $\mathrm{Csp}$ & COF2 & 0.74 & 1 & 1 \\
\hline 34 & $\mathrm{Csp} \mathrm{sp}^{3}$ & CF3 & -2.85 & 11 & 9 \\
\hline 35 & $C \mathrm{sp}^{3}$ & $\mathrm{CF} 2 \mathrm{Cl}$ & -3.44 & 3 & 2 \\
\hline 36 & $\mathrm{Csp}$ & $\mathrm{CFCl} 2$ & -12.04 & 1 & 1 \\
\hline 37 & $\mathrm{Csp}$ & $\mathrm{CCl} 3$ & -12.64 & 2 & 2 \\
\hline 38 & $\mathrm{Csp} \mathrm{sp}^{2}$ & $\mathrm{H} 2=\mathrm{C}$ & -2.93 & 15 & 13 \\
\hline 39 & $\mathrm{Csp} \mathrm{sp}^{2}$ & $\mathrm{HC}=\mathrm{C}$ & -2.16 & 26 & 20 \\
\hline 40 & $C \mathrm{sp}^{2}$ & $\mathrm{HC}=\mathrm{O}$ & -16.45 & 9 & 9 \\
\hline 41 & $\mathrm{Csp}$ & $\mathrm{H}=\mathrm{CN}$ & -13.78 & 17 & 13 \\
\hline 42 & $C \mathrm{sp}^{2}$ & $\mathrm{H}=\mathrm{CO}$ & -10.21 & 1 & 1 \\
\hline 43 & $C \mathrm{sp}^{2}$ & $\mathrm{H}=\mathrm{CS}$ & -6.13 & 2 & 1 \\
\hline 44 & $C \mathrm{sp}^{2}$ & $\mathrm{H}=\mathrm{CCl}$ & -7.34 & 5 & 3 \\
\hline 45 & $C \mathrm{sp}^{2}$ & $\mathrm{HN}=\mathrm{N}$ & -10.70 & 2 & 2 \\
\hline 46 & $\mathrm{Csp} \mathrm{sp}^{2}$ & $\mathrm{HN}=\mathrm{O}$ & -33.05 & 4 & 4 \\
\hline 47 & $\mathrm{Csp} \mathrm{sp}^{2}$ & $\mathrm{HO}=\mathrm{O}$ & -14.45 & 7 & 7 \\
\hline 48 & $C \mathrm{sp}^{2}$ & $\mathrm{C} 2=\mathrm{C}$ & 1.28 & 11 & 11 \\
\hline 49 & $\mathrm{Csp}$ & $\mathrm{C}=\mathrm{CN}$ & -15.51 & 2 & 2 \\
\hline 50 & $\mathrm{Csp} \mathrm{sp}^{2}$ & $\mathrm{C}=\mathrm{CN}(+)$ & -39.48 & 1 & 1 \\
\hline 51 & $C \mathrm{sp}^{2}$ & $\mathrm{C} 2=\mathrm{O}$ & -17.65 & 20 & 20 \\
\hline 52 & $C \mathrm{sp}^{2}$ & $\mathrm{C}=\mathrm{CF}$ & -6.97 & 2 & 2 \\
\hline 53 & $\mathrm{Csp}^{2}$ & $\mathrm{C}=\mathrm{CCl}$ & -31.39 & 1 & 1 \\
\hline 54 & $\mathrm{Csp} \mathrm{sp}^{2}$ & $\mathrm{C}=\mathrm{CBr}$ & -28.79 & 1 & 1 \\
\hline 55 & $\mathrm{Csp} \mathrm{sp}^{2}$ & $\mathrm{C}=\mathrm{CJ}$ & -31.42 & 1 & 1 \\
\hline 56 & $C \mathrm{sp}^{2}$ & $=\mathrm{CN} 2$ & -32.45 & 3 & 3 \\
\hline 57 & $\mathrm{Csp}$ & $\mathrm{CN}=\mathrm{O}$ & -39.35 & 30 & 30 \\
\hline 58 & $\mathrm{Csp} \mathrm{sp}^{2}$ & $=\mathrm{CNCl}$ & -30.33 & 1 & 1 \\
\hline 59 & $C \mathrm{sp}^{2}$ & $\mathrm{CO}=\mathrm{O}$ & -17.24 & 63 & 52 \\
\hline 60 & $\mathrm{Csp}{ }^{2}$ & $=\mathrm{CF} 2$ & 0.44 & 3 & 2 \\
\hline 61 & $\mathrm{Csp} \mathrm{sp}^{2}$ & $=\mathrm{CCl} 2$ & -11.89 & 2 & 2 \\
\hline 62 & $\mathrm{Csp} \mathrm{sp}^{2}$ & $\mathrm{~N} 2=\mathrm{O}$ & -35.29 & 25 & 25 \\
\hline 63 & $C \mathrm{sp}^{2}$ & $\mathrm{~N} 2=\mathrm{S}$ & -41.79 & 6 & 6 \\
\hline 64 & C aromatic & $\mathrm{H}: \mathrm{C} 2$ & -2.84 & 437 & 100 \\
\hline 65 & C aromatic & $\mathrm{H}: \mathrm{C}: \mathrm{N}$ & -14.82 & 29 & 18 \\
\hline 66 & C aromatic & :C3 & -3.23 & 13 & 6 \\
\hline
\end{tabular}


Table 3. Cont

\begin{tabular}{|c|c|c|c|c|c|}
\hline Entry & Atom Type & Neighbours & Contribution & Occurrences & Molecules \\
\hline 67 & $\mathrm{C}$ aromatic & $\mathrm{C}: \mathrm{C} 2$ & -1.72 & 90 & 63 \\
\hline 68 & $\mathrm{C}$ aromatic & $\mathrm{C}: \mathrm{C}: \mathrm{N}$ & -15.13 & 7 & 6 \\
\hline 69 & C aromatic & $: \mathrm{C} 2 \mathrm{~N}$ & -10.35 & 13 & 13 \\
\hline 70 & $\mathrm{C}$ aromatic & $: \mathrm{C} 2 \mathrm{~N}(+)$ & -21.83 & 6 & 6 \\
\hline 71 & $\mathrm{C}$ aromatic & $: \mathrm{C} 2: \mathrm{N}$ & -15.19 & 1 & 1 \\
\hline 72 & $\mathrm{C}$ aromatic & $\mathrm{C} 2 \mathrm{O}$ & -9.63 & 21 & 17 \\
\hline 73 & $\mathrm{C}$ aromatic & $: \mathrm{C} 2 \mathrm{~F}$ & -1.79 & 1 & 1 \\
\hline 74 & $\mathrm{C}$ aromatic & $: \mathrm{C} 2 \mathrm{Cl}$ & -3.91 & 37 & 19 \\
\hline 75 & $\mathrm{C}$ aromatic & :C2Br & -5.99 & 1 & 1 \\
\hline 76 & $\mathrm{C}$ aromatic & $: \mathrm{CN}: \mathrm{N}$ & -16.20 & 1 & 1 \\
\hline 77 & $\mathrm{Csp}$ & $\mathrm{H \# C}$ & -1.37 & 1 & 1 \\
\hline 78 & C sp & C\#C & 0.00 & 1 & 1 \\
\hline 79 & C sp & $\mathrm{C \# N}$ & -17.66 & 15 & 12 \\
\hline 80 & $\mathrm{Nsp}^{3}$ & $\mathrm{H} 2 \mathrm{C}$ & -2.40 & 25 & 20 \\
\hline 81 & $\mathrm{Nsp}^{3}$ & $\mathrm{H} 2 \mathrm{C}(\mathrm{pi})$ & -16.13 & 32 & 30 \\
\hline 82 & $\mathrm{~N} \mathrm{sp}^{3}$ & HC2 & 24.30 & 6 & 6 \\
\hline 83 & $\mathrm{~N} \mathrm{sp}^{3}$ & HC2(pi) & 11.97 & 26 & 22 \\
\hline 84 & $\mathrm{~N} \mathrm{sp}^{3}$ & $\mathrm{HC} 2(2 \mathrm{pi})$ & 3.09 & 21 & 12 \\
\hline 85 & $\mathrm{~N} \mathrm{sp}^{3}$ & $\mathrm{C} 3$ & 57.51 & 5 & 5 \\
\hline 86 & $\mathrm{~N} \mathrm{sp}$ & C3(pi) & 52.51 & 10 & 9 \\
\hline 87 & $\mathrm{~N} \mathrm{sp}$ & C3(2pi) & 36.53 & 13 & 8 \\
\hline 88 & $\mathrm{~N} \mathrm{sp}^{2}$ & $\mathrm{C}=\mathrm{C}$ & -19.81 & 2 & 2 \\
\hline 89 & $\mathrm{~N}$ aromatic & :C2 & 5.38 & 19 & 19 \\
\hline 90 & $\mathrm{~N}(+) \mathrm{sp}^{2}$ & $\mathrm{CO}=\mathrm{O}(-)$ & 8.85 & 11 & 11 \\
\hline 91 & $\mathrm{O}$ & $\mathrm{HC}$ & -17.23 & 61 & 50 \\
\hline 92 & $\mathrm{O}$ & $\mathrm{HC}(\mathrm{pi})$ & -18.29 & 32 & 26 \\
\hline 93 & $\mathrm{O}$ & $\mathrm{HO}$ & -22.54 & 2 & 1 \\
\hline 94 & $\mathrm{O}$ & $\mathrm{C} 2$ & 8.60 & 68 & 39 \\
\hline 95 & $\mathrm{O}$ & C2(pi) & 10.97 & 56 & 49 \\
\hline 96 & $\mathrm{O}$ & C2(2pi) & 9.97 & 2 & 2 \\
\hline 97 & S2 & $\mathrm{HC}$ & 1.98 & 4 & 4 \\
\hline 98 & S2 & $\mathrm{C} 2$ & 6.62 & 3 & 3 \\
\hline 99 & S2 & C2(2pi) & 0.00 & 1 & 1 \\
\hline 100 & S2 & CS & 2.30 & 4 & 2 \\
\hline 101 & S4 & $\mathrm{C} 2=\mathrm{O}$ & -33.00 & 1 & 1 \\
\hline 102 & $\mathrm{H}$ & H Acceptor & 10.02 & 2 & 2 \\
\hline 103 & Alkane & No. of C atoms & 0.96 & 142 & 23 \\
\hline 104 & Unsaturated HC & No. of $\mathrm{C}$ atoms & 0.25 & 307 & 37 \\
\hline $\mathrm{A}$ & Based on & Valid groups & 61 & & 436 \\
\hline $\mathrm{B}$ & Goodness of fit & $R^{2}$ & 0.9731 & & 388 \\
\hline $\mathrm{C}$ & Deviation & Average & 2.68 & & 388 \\
\hline $\mathrm{D}$ & Deviation & Standard & 3.53 & & 388 \\
\hline $\mathrm{E}$ & K-fold cv & K & 10 & & 373 \\
\hline $\mathrm{F}$ & Goodness of fit & $Q^{2}$ & 0.9546 & & 373 \\
\hline G & Deviation & Average (cv) & 3.22 & & 373 \\
\hline $\mathrm{H}$ & Deviation & Standard (cv) & 4.34 & & 373 \\
\hline
\end{tabular}

Due to the limited number of compounds, the histogram in Figures 7 and 8 reveals a slightly distorted Gaussian bell form. Nevertheless, the analysis of the error distribution reveals that $78.8 \%$ of the compounds deviated by less than one $\mathrm{cv}$-standard deviation, whereas for only $5 \%$ the deviation was larger than twice this value. The small number of only 61 valid atom groups limited the range of compounds in the database eligible for a heat-of-solvation prediction to $40 \%$. As an informational note, the Abraham model used by Mintz et al. [69] described the enthalpy-of-solvation data of the 369 compounds in their data set to within a standard deviation of $4.04 \mathrm{~kJ} / \mathrm{mol}$, which is slightly larger than our standard deviation of $3.53 \mathrm{~kJ} / \mathrm{mol}$ based on a data set of 388 compounds. Beyond this, of the 
thermodynamic properties considered here and in the previous paper [1], the Abraham model can only predict enthalpies of solvation.

The observant reader may have noticed that the goodness of fit of the heat-of-solvation calculation is better than that for the heat of sublimation, although the experimental source for the former is the difference between the heat of solution and the heat of sublimation (or vaporization). Hence, one would expect that the uncertainty of the heat of sublimation would be reflected in the goodness of fit of the heat of solvation. The reason as to why this is not the case lies in the nature of the experimental measurements which reduces the chemical diversity: while the determination of the heat of sublimation in principle allows molecules of nearly any size and complexity, the solvation experiments are limited to mostly simple organic liquids and solids having only one, two or three functional groups because these molecules had to exhibit sufficient solubility in water to enable the measurement and they had to readily dissolve within a reasonable amount of time. This precondition eliminated compounds with poor water solubility, in other words many of the larger species. These limitations are also visible in the scope of the experimental enthalpy values: while the range for the heat of sublimation is between about 30 and $330 \mathrm{~kJ} / \mathrm{mol}$, for the heat of solvation it is only between about -12 and $-150 \mathrm{~kJ} / \mathrm{mol}$, i.e., much smaller. It is reasonable to presume that if the solvation experiments would include structurally complex compounds, the correlation deviations would be larger. This size limitation has also a negative effect on the diversity of the atom groups, as can be seen in Table 3, row A, where the number of "valid" groups, available for the calculation of the heat of solvation, is only 61 in relation to 154 (see Table 2, row A) for the heat of sublimation.

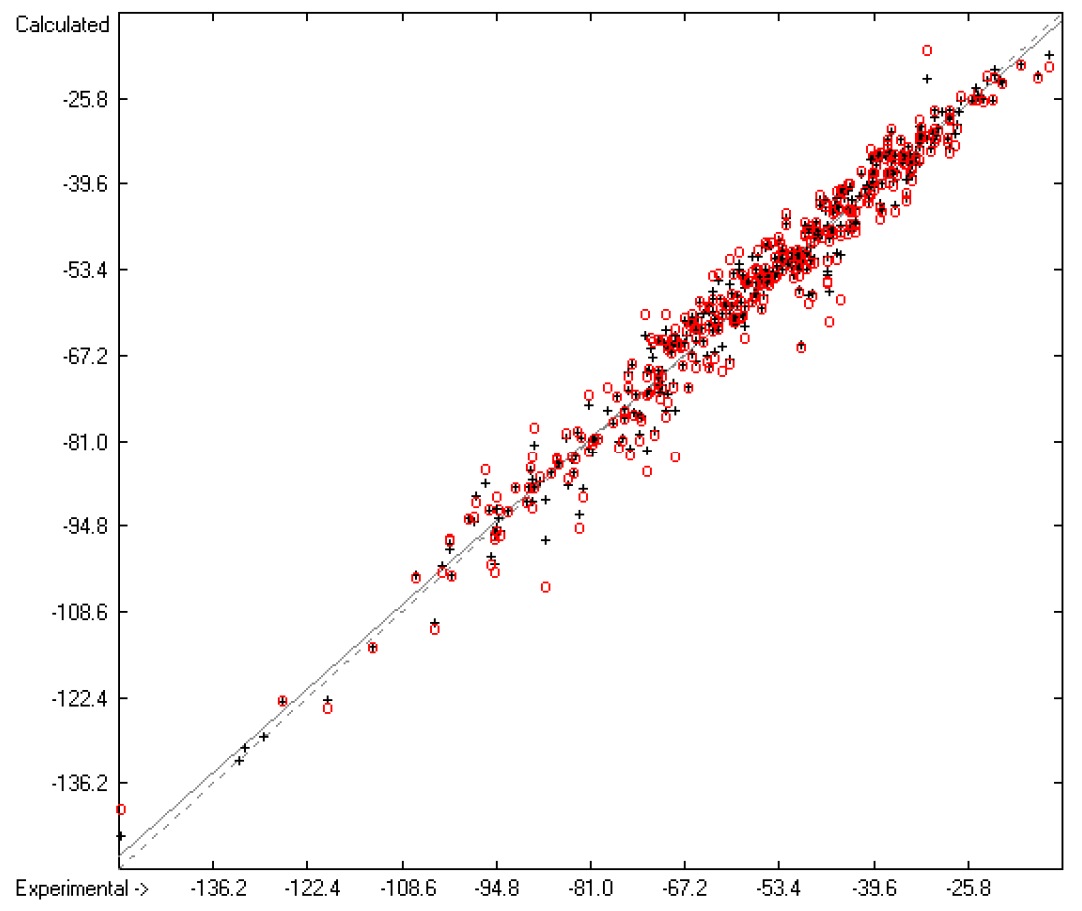

Figure 7. Correlation Diagram of the Enthalpy-of-Solvation Data $\left(N=388 ; R^{2}=0.9731 ; Q^{2}=0.9546\right.$; regression line: intercept $=-1.4422$, slope $=0.9759$ ). 


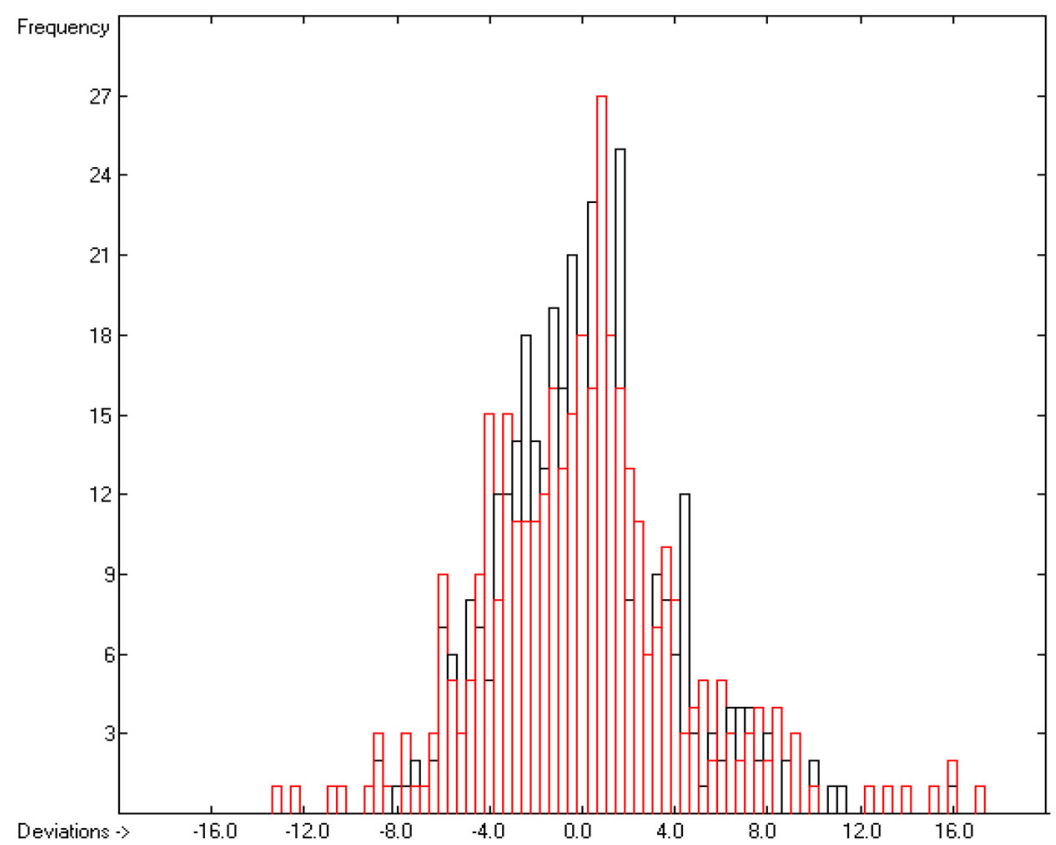

Figure 8. Histogram of the Enthalpy-of-Solvation Data $(S=4.34 \mathrm{~kJ} / \mathrm{mol}$; exp. values range: -149.51--13.7 kJ/mol).

\subsection{Entropy of Fusion}

The entropy of fusion under this subtitle is defined as the entropy change associated with the phase change from the crystalline to the isotropic liquid state of a molecule without passing any intermediate anisotropic, semiliquid phases. In most cases this transition indeed occurs in one stage, but several molecules, on addition of heat, undergo a change from one crystalline phase to a second or even third energetically less stable phase prior to melting. In the following, the entropy-of-fusion values cited in the tables are the sum of all the entropies associated with these solid-solid phase changes including the final solid-liquid phase change. The main source for these values was found in the comprehensive collection of Chickos, Acree and Liebman [21] and in its update [60]. More recent entropy-of-fusion data were found for long-chain fluorinated alcohols [33], halogenated fluorenes [35], di- and tri(cyanatophenyl)alkanes and -silanes [62], 2-cyano-4'-methylbiphenyl [83], diphenyl cyclohexyl-phosphoramidate [84] and 3,4-dinitrofurazanfuroxan [85]. The complete set of compounds with experimental entropy-of-fusion values amounted to a total of 2809 used for the evaluation of the atom-group parameters, yielding 188 valid atom groups. Various tentative calculations including or excluding certain special groups revealed a distinct improvement of the goodness of fit of the optimization process, if the group "Endocyclic bonds" was involved, which counts all single endocyclic bonds in a molecule. However, for small molecules containing small rings this group parameter tended to overcompensate the decrease of freedom of mobility and, therefore, the three special groups "Angle60", "Angle90" and "Angle102" were added as counter-correctives. The cross-validation calculation with 2637 samples resulted in a very satisfying goodness of fit $Q^{2}$ of 0.8727 and a standard deviation of $17.93 \mathrm{~J} / \mathrm{mol} / \mathrm{K}$. In Table 4 the results of these calculations are summarized. Fifty-five compounds had to be removed from the calculations as their experimental values deviated from prediction by more than three times the cv-standard deviation. The large number of valid atom groups, on the other hand, enabled the calculation of the entropy of fusion for $81.8 \%$ of the database's compounds. 
Table 4. Atom Groups and their Contributions (in J/mol/K) for Entropy-of-Fusion Calculations.

\begin{tabular}{|c|c|c|c|c|c|}
\hline Entry & Atom Type & Neighbours & Contribution & Occurrences & Molecules \\
\hline 1 & Const & & 31.12 & 2809 & 2809 \\
\hline 2 & B & $\mathrm{C} 3$ & 12.34 & 2 & 2 \\
\hline 3 & B & $\mathrm{CO} 2$ & 51.11 & 5 & 5 \\
\hline 4 & $\mathrm{Csp} \mathrm{sp}^{3}$ & $\mathrm{H} 3 \mathrm{~B}$ & -4.93 & 3 & 1 \\
\hline 5 & $\mathrm{Csp} \mathrm{sp}^{3}$ & $\mathrm{H} 2 \mathrm{BC}$ & 4.93 & 3 & 1 \\
\hline 6 & $\mathrm{Csp}$ & Н3С & 1.90 & 2944 & 1402 \\
\hline 7 & $\mathrm{Csp}$ & $\mathrm{H} 3 \mathrm{~N}$ & 15.63 & 279 & 149 \\
\hline 8 & $\mathrm{Csp}$ & $\mathrm{H} 3 \mathrm{~N}(+)$ & 7.07 & 2 & 2 \\
\hline 9 & $\mathrm{Csp}$ & $\mathrm{H} 3 \mathrm{O}$ & 14.42 & 366 & 232 \\
\hline 10 & $\mathrm{C} \mathrm{sp}^{3}$ & $\mathrm{H} 3 \mathrm{P}$ & 21.07 & 3 & 3 \\
\hline 11 & $C \mathrm{sp}^{3}$ & H3S & 12.93 & 35 & 31 \\
\hline 12 & $\mathrm{Csp} \mathrm{sp}^{3}$ & $\mathrm{H} 3 \mathrm{Si}$ & 8.19 & 283 & 46 \\
\hline 13 & $\mathrm{Csp}$ & $\mathrm{H} 2 \mathrm{C} 2$ & 8.46 & 8600 & 1239 \\
\hline 14 & $\mathrm{Csp}$ & $\mathrm{H} 2 \mathrm{CN}$ & 14.85 & 505 & 257 \\
\hline 15 & $\mathrm{Csp}$ & $\mathrm{H} 2 \mathrm{CN}(+)$ & 19.09 & 29 & 21 \\
\hline 16 & $\mathrm{Csp}$ & $\mathrm{H} 2 \mathrm{CO}$ & 14.52 & 952 & 473 \\
\hline 17 & $C \mathrm{sp}^{3}$ & $\mathrm{H} 2 \mathrm{CP}$ & 17.50 & 3 & 2 \\
\hline 18 & $\mathrm{Csp}$ & $\mathrm{H} 2 \mathrm{CS}$ & 16.77 & 166 & 83 \\
\hline 19 & $\mathrm{Csp}$ & $\mathrm{H} 2 \mathrm{CF}$ & 12.36 & 1 & 1 \\
\hline 20 & $\mathrm{Csp}$ & $\mathrm{H} 2 \mathrm{CCl}$ & 10.67 & 30 & 24 \\
\hline 21 & $\mathrm{Csp}$ & $\mathrm{H} 2 \mathrm{CBr}$ & 11.79 & 24 & 17 \\
\hline 22 & $\mathrm{Csp} \mathrm{sp}^{3}$ & $\mathrm{H} 2 \mathrm{CJ}$ & 3.10 & 2 & 2 \\
\hline 23 & $\mathrm{Csp} \mathrm{sp}^{3}$ & $\mathrm{H} 2 \mathrm{CSi}$ & 8.50 & 62 & 20 \\
\hline 24 & $C \mathrm{sp}^{3}$ & $\mathrm{H} 2 \mathrm{~N} 2$ & 5.03 & 20 & 11 \\
\hline 25 & $C \mathrm{sp}^{3}$ & $\mathrm{H} 2 \mathrm{NO}$ & 8.98 & 8 & 7 \\
\hline 26 & $\mathrm{C} \mathrm{sp}^{3}$ & $\mathrm{H} 2 \mathrm{NS}$ & 43.70 & 4 & 4 \\
\hline 27 & $\mathrm{C} \mathrm{sp}^{3}$ & $\mathrm{H} 2 \mathrm{O} 2$ & 22.34 & 23 & 14 \\
\hline 28 & $C \mathrm{sp}^{3}$ & $\mathrm{H} 2 \mathrm{~S} 2$ & 29.21 & 7 & 5 \\
\hline 29 & $\mathrm{Csp} \mathrm{sp}^{3}$ & $\mathrm{H} 2 \mathrm{SCl}$ & 22.89 & 1 & 1 \\
\hline 30 & $C \mathrm{sp}^{3}$ & $\mathrm{H} 2 \mathrm{Si} 2$ & 12.02 & 6 & 3 \\
\hline 31 & $\mathrm{Csp} \mathrm{sp}^{3}$ & HC3 & 0.64 & 817 & 388 \\
\hline 32 & $\mathrm{Csp} \mathrm{sp}^{3}$ & $\mathrm{HC} 2 \mathrm{~N}$ & 18.09 & 117 & 103 \\
\hline 33 & $\mathrm{Csp}$ & $\mathrm{HC} 2 \mathrm{~N}(+)$ & -9.91 & 16 & 16 \\
\hline 34 & $C \mathrm{sp}^{3}$ & $\mathrm{HC} 2 \mathrm{O}$ & 10.63 & 357 & 226 \\
\hline 35 & $\mathrm{Csp}$ & $\mathrm{HC} 2 \mathrm{~S}$ & 9.80 & 18 & 13 \\
\hline 36 & $C \mathrm{sp}^{3}$ & $\mathrm{HC} 2 \mathrm{~F}$ & 8.23 & 2 & 2 \\
\hline 37 & $C \mathrm{sp}^{3}$ & $\mathrm{HC} 2 \mathrm{Cl}$ & 10.38 & 22 & 10 \\
\hline 38 & $\mathrm{Csp}$ & $\mathrm{HC} 2 \mathrm{Br}$ & 8.94 & 5 & 4 \\
\hline 39 & $C \mathrm{sp}^{3}$ & $\mathrm{HC} 2 \mathrm{Si}$ & -14.02 & 1 & 1 \\
\hline 40 & $C \mathrm{sp}^{3}$ & HCN2 & 1.21 & 2 & 1 \\
\hline 41 & $\mathrm{Csp} \mathrm{sp}^{3}$ & $\mathrm{HCNO}$ & 23.14 & 7 & 6 \\
\hline 42 & $\mathrm{Csp} \mathrm{sp}^{3}$ & HCNS & 23.70 & 1 & 1 \\
\hline 43 & $C \mathrm{sp}^{3}$ & $\mathrm{HCO} 2$ & 19.18 & 30 & 26 \\
\hline 44 & $\mathrm{Csp} \mathrm{sp}^{3}$ & $\mathrm{HCOCl}$ & 19.13 & 2 & 1 \\
\hline 45 & $C \mathrm{sp}^{3}$ & HCF2 & 4.20 & 4 & 4 \\
\hline 46 & $\mathrm{Csp} \mathrm{sp}^{3}$ & $\mathrm{HCFCl}$ & -10.16 & 1 & 1 \\
\hline 47 & $\mathrm{Csp} \mathrm{sp}^{3}$ & $\mathrm{HCCl} 2$ & 9.01 & 10 & 9 \\
\hline 48 & $\mathrm{Csp}$ & $\mathrm{HCClBr}$ & -3.80 & 1 & 1 \\
\hline 49 & $\mathrm{Csp} \mathrm{sp}^{3}$ & C4 & -0.23 & 435 & 256 \\
\hline 50 & $\mathrm{Csp}{ }^{3}$ & $\mathrm{C} 3 \mathrm{~N}$ & 14.87 & 22 & 20 \\
\hline 51 & $C \mathrm{sp}^{3}$ & $\mathrm{C} 3 \mathrm{~N}(+)$ & 12.86 & 6 & 5 \\
\hline 52 & $\mathrm{Csp}$ & $\mathrm{C} 3 \mathrm{O}$ & 4.63 & 81 & 74 \\
\hline 53 & $C \mathrm{sp}^{3}$ & C3S & 16.54 & 6 & 6 \\
\hline 54 & $\mathrm{Csp} \mathrm{sp}^{3}$ & $\mathrm{C} 3 \mathrm{~F}$ & 18.64 & 14 & 12 \\
\hline 55 & $\mathrm{Csp}$ & $\mathrm{C} 3 \mathrm{Cl}$ & 9.23 & 14 & 9 \\
\hline 56 & $C \mathrm{sp}^{3}$ & $\mathrm{C} 3 \mathrm{Br}$ & 3.44 & 2 & 2 \\
\hline 57 & $\mathrm{Csp} \mathrm{sp}^{3}$ & $\mathrm{C} 3 \mathrm{~J}$ & 31.10 & 1 & 1 \\
\hline
\end{tabular}


Table 4. Cont.

\begin{tabular}{|c|c|c|c|c|c|}
\hline Entry & Atom Type & Neighbours & Contribution & Occurrences & Molecules \\
\hline 58 & $\mathrm{Csp}^{3}$ & $\mathrm{C} 2 \mathrm{~N} 2$ & 52.69 & 3 & 2 \\
\hline 59 & $\mathrm{Csp}^{3}$ & C2N2(+) & 4.24 & 7 & 6 \\
\hline 60 & $\mathrm{C} \mathrm{sp}^{3}$ & $\mathrm{C} 2 \mathrm{NO}$ & 34.66 & 1 & 1 \\
\hline 61 & $\mathrm{Csp}$ & $\mathrm{C} 2 \mathrm{NF}$ & 47.27 & 1 & 1 \\
\hline 62 & $\mathrm{C} \mathrm{sp}^{3}$ & $\mathrm{C} 2 \mathrm{NCl}(+)$ & 13.35 & 1 & 1 \\
\hline 63 & $C \mathrm{sp}^{3}$ & $\mathrm{C} 2 \mathrm{O} 2$ & 13.44 & 47 & 29 \\
\hline 64 & $\mathrm{Csp}$ & $\mathrm{C} 2 \mathrm{~S} 2$ & 10.13 & 1 & 1 \\
\hline 65 & $\mathrm{Csp}$ & $\mathrm{C} 2 \mathrm{~F} 2$ & -0.09 & 262 & 37 \\
\hline 66 & $\mathrm{C} \mathrm{sp}^{3}$ & $\mathrm{C} 2 \mathrm{Cl} 2$ & 10.32 & 9 & 7 \\
\hline 67 & $\mathrm{C} \mathrm{sp}^{3}$ & $\mathrm{CN} 3(+)$ & 7.29 & 6 & 5 \\
\hline 68 & $\mathrm{Csp}$ & CNF2 & 6.86 & 7 & 3 \\
\hline 69 & $\mathrm{Csp}$ & COF2 & -3.57 & 4 & 3 \\
\hline 70 & $\mathrm{Csp}$ & CS3 & 30.56 & 4 & 1 \\
\hline 71 & $\mathrm{Csp}$ & CSF2 & 41.61 & 2 & 1 \\
\hline 72 & $C \mathrm{sp}^{3}$ & $\mathrm{CSCl} 2$ & 46.90 & 2 & 2 \\
\hline 73 & $\mathrm{C} \mathrm{sp}^{3}$ & CF3 & 3.38 & 91 & 76 \\
\hline 74 & $\mathrm{C} \mathrm{sp}^{3}$ & $\mathrm{CF} 2 \mathrm{Cl}$ & -1.55 & 6 & 5 \\
\hline 75 & $\mathrm{C} \mathrm{sp}^{3}$ & $\mathrm{CF} 2 \mathrm{Br}$ & 8.94 & 4 & 3 \\
\hline 76 & $\mathrm{Csp}$ & $\mathrm{CFCl} 2$ & -6.89 & 3 & 2 \\
\hline 77 & $\mathrm{C} \mathrm{sp}^{3}$ & $\mathrm{CCl} 3$ & 0.92 & 17 & 16 \\
\hline 78 & $\mathrm{C} \mathrm{sp}^{3}$ & NF3 & 11.04 & 1 & 1 \\
\hline 79 & $\mathrm{C} \mathrm{sp}^{3}$ & $\mathrm{O} 2 \mathrm{~F} 2$ & 20.23 & 1 & 1 \\
\hline 80 & $\mathrm{C} \mathrm{sp}^{3}$ & OF3 & 2.25 & 2 & 2 \\
\hline 81 & $\mathrm{C} \mathrm{sp}^{3}$ & SF3 & 24.96 & 4 & 4 \\
\hline 82 & $\mathrm{Csp}{ }^{3}$ & $\mathrm{SCl} 3$ & 46.90 & 1 & 1 \\
\hline 83 & $\mathrm{C} \mathrm{sp}^{3}$ & $\mathrm{SiCl} 3$ & 14.20 & 1 & 1 \\
\hline 84 & $C \mathrm{sp}^{2}$ & $\mathrm{H} 2=\mathrm{C}$ & 5.49 & 84 & 76 \\
\hline 85 & $C \mathrm{sp}^{2}$ & $\mathrm{HC}=\mathrm{C}$ & 2.46 & 607 & 323 \\
\hline 86 & $C \mathrm{sp}^{2}$ & $\mathrm{HC}=\mathrm{N}$ & -0.81 & 48 & 40 \\
\hline 87 & $C \mathrm{sp}^{2}$ & $\mathrm{H}=\mathrm{CN}$ & 3.18 & 44 & 37 \\
\hline 88 & $C \mathrm{sp}^{2}$ & $\mathrm{HC}=\mathrm{O}$ & 8.29 & 18 & 18 \\
\hline 89 & $C \mathrm{sp}^{2}$ & $\mathrm{H}=\mathrm{CO}$ & 5.29 & 19 & 17 \\
\hline 90 & $C \mathrm{sp}^{2}$ & $\mathrm{H}=\mathrm{CS}$ & -1.85 & 43 & 33 \\
\hline 91 & $C \mathrm{sp}^{2}$ & $\mathrm{H}=\mathrm{CCl}$ & 10.11 & 3 & 3 \\
\hline 92 & $C \mathrm{sp}^{2}$ & $\mathrm{H}=\mathrm{CSi}$ & 2.92 & 3 & 3 \\
\hline 93 & $C \mathrm{sp}^{2}$ & $\mathrm{HN}=\mathrm{N}$ & 9.78 & 30 & 22 \\
\hline 94 & $\mathrm{C} \mathrm{sp}^{2}$ & $\mathrm{HN}=\mathrm{O}$ & -10.25 & 3 & 3 \\
\hline 95 & $C \mathrm{sp}^{2}$ & $\mathrm{H}=\mathrm{NO}$ & 21.94 & 1 & 1 \\
\hline 96 & $\mathrm{C} \mathrm{sp}^{2}$ & $\mathrm{H}=\mathrm{NS}$ & 1.04 & 4 & 4 \\
\hline 97 & $\mathrm{C} \mathrm{sp}^{2}$ & $\mathrm{HO}=\mathrm{O}$ & 14.63 & 2 & 2 \\
\hline 98 & $\mathrm{C} \mathrm{sp}^{2}$ & $\mathrm{C} 2=\mathrm{C}$ & 0.30 & 212 & 166 \\
\hline 99 & $C \mathrm{sp}^{2}$ & $\mathrm{C} 2=\mathrm{N}$ & 7.33 & 35 & 33 \\
\hline 100 & $\mathrm{C} \mathrm{sp}^{2}$ & $\mathrm{C} 2=\mathrm{N}(+)$ & 2.31 & 1 & 1 \\
\hline 101 & $C \mathrm{sp}^{2}$ & $\mathrm{C}=\mathrm{CN}$ & -2.70 & 51 & 45 \\
\hline 102 & $C \mathrm{sp}^{2}$ & $\mathrm{C}=\mathrm{CN}(+)$ & 0.00 & 2 & 1 \\
\hline 103 & $\mathrm{C} \mathrm{sp}^{2}$ & $\mathrm{C} 2=\mathrm{O}$ & 1.57 & 386 & 298 \\
\hline 104 & $\mathrm{Csp}{ }^{2}$ & $\mathrm{C}=\mathrm{CO}$ & 5.58 & 70 & 52 \\
\hline 105 & $C \mathrm{sp}^{2}$ & $\mathrm{C}=\mathrm{CS}$ & 0.18 & 38 & 25 \\
\hline 106 & $C \mathrm{sp}^{2}$ & $\mathrm{C}=\mathrm{CCl}$ & 3.68 & 20 & 13 \\
\hline 107 & $C \mathrm{sp}^{2}$ & $\mathrm{C}=\mathrm{CBr}$ & 45.90 & 1 & 1 \\
\hline 108 & $C \mathrm{sp}^{2}$ & $=\mathrm{CN} 2$ & 12.85 & 17 & 17 \\
\hline 109 & $C \mathrm{sp}^{2}$ & $=\mathrm{CN} 2(+)$ & 6.14 & 1 & 1 \\
\hline 110 & $C \mathrm{sp}^{2}$ & $\mathrm{CN}=\mathrm{N}$ & 1.47 & 25 & 19 \\
\hline 111 & $C \mathrm{sp}^{2}$ & $=\mathrm{CNO}$ & -1.47 & 6 & 4 \\
\hline 112 & $C \mathrm{sp}^{2}$ & $\mathrm{CN}=\mathrm{O}$ & 0.63 & 366 & 234 \\
\hline 113 & $C \mathrm{sp}^{2}$ & $\mathrm{C}=\mathrm{NO}$ & 9.33 & 5 & 5 \\
\hline 114 & $C \mathrm{sp}^{2}$ & $\mathrm{C}=\mathrm{NS}$ & 7.20 & 7 & 7 \\
\hline
\end{tabular}


Table 4. Cont.

\begin{tabular}{|c|c|c|c|c|c|}
\hline Entry & Atom Type & Neighbours & Contribution & Occurrences & Molecules \\
\hline 115 & $\mathrm{Csp}^{2}$ & $\mathrm{CN}=\mathrm{S}$ & -2.87 & 10 & 8 \\
\hline 116 & $\mathrm{C} \mathrm{sp}^{2}$ & $=\mathrm{CNCl}$ & 11.25 & 1 & 1 \\
\hline 117 & $\mathrm{Csp}^{2}$ & $\mathrm{CO}=\mathrm{O}$ & 5.68 & 718 & 546 \\
\hline 118 & $C \mathrm{sp}^{2}$ & $\mathrm{CO}=\mathrm{O}(-)$ & -16.84 & 19 & 19 \\
\hline 119 & $\mathrm{C} \mathrm{sp}^{2}$ & $\mathrm{C}=\mathrm{OF}$ & 9.78 & 3 & 2 \\
\hline 120 & $C \mathrm{sp}^{2}$ & $\mathrm{C}=\mathrm{OCl}$ & 14.97 & 2 & 1 \\
\hline 121 & $\mathrm{C} \mathrm{sp}^{2}$ & $\mathrm{C}=\mathrm{OS}$ & 16.72 & 1 & 1 \\
\hline 122 & $\mathrm{C} \mathrm{sp}^{2}$ & $=\mathrm{CS} 2$ & -7.29 & 12 & 2 \\
\hline 123 & $\mathrm{Csp}^{2}$ & $=\mathrm{CSCl}$ & 2.93 & 3 & 2 \\
\hline 124 & $C \mathrm{sp}^{2}$ & $=\mathrm{CSBr}$ & -4.03 & 1 & 1 \\
\hline 125 & $C \mathrm{sp}^{2}$ & $=\mathrm{CF} 2$ & 11.60 & 3 & 2 \\
\hline 126 & $C \mathrm{sp}^{2}$ & $=\mathrm{CFCl}$ & 1.87 & 1 & 1 \\
\hline 127 & $\mathrm{C} \mathrm{sp}^{2}$ & $=\mathrm{CCl} 2$ & 5.32 & 9 & 8 \\
\hline 128 & $\mathrm{C} \mathrm{sp}^{2}$ & $=\mathrm{CBr} 2$ & 46.05 & 1 & 1 \\
\hline 129 & $C \mathrm{sp}^{2}$ & $\mathrm{~N} 2=\mathrm{N}$ & 11.87 & 9 & 9 \\
\hline 130 & $\mathrm{C} \mathrm{sp}^{2}$ & $\mathrm{~N} 2=\mathrm{O}$ & -3.48 & 90 & 84 \\
\hline 131 & $\mathrm{C} \mathrm{sp}^{2}$ & $\mathrm{~N}=\mathrm{NO}$ & 3.41 & 1 & 1 \\
\hline 132 & $\mathrm{C} \mathrm{sp}^{2}$ & $\mathrm{~N} 2=\mathrm{S}$ & 0.55 & 32 & 31 \\
\hline 133 & $C \mathrm{sp}^{2}$ & $\mathrm{~N}=\mathrm{NS}$ & -3.08 & 23 & 23 \\
\hline 134 & $\mathrm{Csp}^{2}$ & $\mathrm{NO}=\mathrm{O}$ & 0.38 & 62 & 60 \\
\hline 135 & $C \mathrm{sp}^{2}$ & $\mathrm{~N}=\mathrm{OS}$ & 20.86 & 2 & 2 \\
\hline 136 & $\mathrm{C} \mathrm{sp}^{2}$ & $\mathrm{NO}=\mathrm{S}$ & -2.08 & 8 & 8 \\
\hline 137 & $\mathrm{C} \mathrm{sp}^{2}$ & $\mathrm{NS}=\mathrm{S}$ & 25.24 & 3 & 3 \\
\hline 138 & $\mathrm{Csp}{ }^{2}$ & $=\mathrm{NS} 2$ & -12.86 & 2 & 2 \\
\hline 139 & $C \mathrm{sp}^{2}$ & $\mathrm{O} 2=\mathrm{O}$ & -9.60 & 10 & 10 \\
\hline 140 & $C \mathrm{sp}^{2}$ & $=\mathrm{OS} 2$ & 6.53 & 1 & 1 \\
\hline 141 & $\mathrm{C}$ aromatic & $\mathrm{B}: \mathrm{C} 2$ & -47.51 & 5 & 5 \\
\hline 142 & $\mathrm{C}$ aromatic & $\mathrm{H}: \mathrm{C} 2$ & 2.57 & 8600 & 1498 \\
\hline 143 & $\mathrm{C}$ aromatic & $\mathrm{H}: \mathrm{C}: \mathrm{N}$ & 1.17 & 108 & 68 \\
\hline 144 & $\mathrm{C}$ aromatic & $\mathrm{H}: \mathrm{N} 2$ & -1.12 & 5 & 3 \\
\hline 145 & $\mathrm{C}$ aromatic & :C3 & -1.60 & 481 & 153 \\
\hline 146 & $\mathrm{C}$ aromatic & $\mathrm{C}: \mathrm{C} 2$ & -2.58 & 2198 & 1062 \\
\hline 147 & $\mathrm{C}$ aromatic & $C: C: N$ & 5.44 & 46 & 38 \\
\hline 148 & C aromatic & $: \mathrm{C} 2 \mathrm{~N}$ & -0.38 & 524 & 389 \\
\hline 149 & $\mathrm{C}$ aromatic & $: \mathrm{C} 2: \mathrm{N}$ & -5.26 & 33 & 20 \\
\hline 150 & $\mathrm{C}$ aromatic & $: \mathrm{C} 2 \mathrm{~N}(+)$ & 4.26 & 203 & 144 \\
\hline 151 & $\mathrm{C}$ aromatic & $: \mathrm{C} 2 \mathrm{O}$ & 2.82 & 853 & 532 \\
\hline 152 & C aromatic & $: \mathrm{C} 2 \mathrm{P}$ & -2.68 & 12 & 5 \\
\hline 153 & C aromatic & :C2S & 0.30 & 98 & 73 \\
\hline 154 & $\mathrm{C}$ aromatic & $: \mathrm{C} 2 \mathrm{Si}$ & 3.80 & 45 & 21 \\
\hline 155 & $\mathrm{C}$ aromatic & :C2F & 4.24 & 150 & 69 \\
\hline 156 & $\mathrm{C}$ aromatic & $: \mathrm{C} 2 \mathrm{Cl}$ & 5.68 & 860 & 318 \\
\hline 157 & $\mathrm{C}$ aromatic & $\mathrm{C} 2 \mathrm{Br}$ & 4.73 & 92 & 57 \\
\hline 158 & $\mathrm{C}$ aromatic & :C2J & 6.30 & 26 & 19 \\
\hline 159 & $\mathrm{C}$ aromatic & $: \mathrm{CN}: \mathrm{N}$ & 5.87 & 28 & 27 \\
\hline 160 & $\mathrm{C}$ aromatic & :CN:N(+) & 0.05 & 2 & 1 \\
\hline 161 & $\mathrm{C}$ aromatic & :C:NO & 3.76 & 9 & 7 \\
\hline 162 & $\mathrm{C}$ aromatic & :C:NS & 2.70 & 2 & 1 \\
\hline 163 & $\mathrm{C}$ aromatic & :C:NCl & 9.38 & 8 & 8 \\
\hline 164 & C aromatic & $\mathrm{N}: \mathrm{N} 2$ & -9.59 & 85 & 40 \\
\hline 165 & $\mathrm{C}$ aromatic & :N2O & -5.16 & 4 & 2 \\
\hline 166 & $\mathrm{C}$ aromatic & :N2S & -2.43 & 5 & 5 \\
\hline 167 & $\mathrm{C}$ aromatic & :N2Cl & 19.07 & 8 & 7 \\
\hline 168 & C sp & $\mathrm{H \# C}$ & 2.83 & 26 & 23 \\
\hline 169 & C sp & $\mathrm{C \# C}$ & -0.52 & 183 & 83 \\
\hline 170 & C sp & $=\mathrm{C} 2$ & 7.54 & 4 & 4 \\
\hline 171 & $\mathrm{Csp}$ & $\mathrm{CHN}$ & 2.66 & 120 & 94 \\
\hline
\end{tabular}


Table 4. Cont.

\begin{tabular}{|c|c|c|c|c|c|}
\hline Entry & Atom Type & Neighbours & Contribution & Occurrences & Molecules \\
\hline 172 & C sp & \#CSi & 3.40 & 3 & 2 \\
\hline 173 & C sp & $\mathrm{N \# N}$ & -16.19 & 1 & 1 \\
\hline 174 & C sp & $=\mathrm{N} 2$ & 23.07 & 1 & 1 \\
\hline 175 & C sp & \#NO & 6.78 & 10 & 4 \\
\hline 176 & C sp & $=\mathrm{N}=\mathrm{O}$ & 14.08 & 6 & 3 \\
\hline 177 & $\mathrm{~N} \mathrm{sp}^{3}$ & $\mathrm{H} 2 \mathrm{C}$ & 9.39 & 34 & 21 \\
\hline 178 & $\mathrm{~N} \mathrm{sp}^{3}$ & $\mathrm{H} 2 \mathrm{C}(\mathrm{pi})$ & 7.89 & 190 & 160 \\
\hline 179 & $\mathrm{~N} \mathrm{sp}^{3}$ & $\mathrm{H} 2 \mathrm{~N}$ & 0.92 & 5 & 5 \\
\hline 180 & $\mathrm{~N} \mathrm{sp}^{3}$ & $\mathrm{H} 2 \mathrm{P}$ & -16.37 & 1 & 1 \\
\hline 181 & $\mathrm{~N} \mathrm{sp}^{3}$ & $\mathrm{H} 2 \mathrm{~S}$ & 10.07 & 7 & 7 \\
\hline 182 & $\mathrm{~N} \mathrm{sp}^{3}$ & $\mathrm{HC} 2$ & -1.65 & 20 & 20 \\
\hline 183 & $\mathrm{~N} \mathrm{sp}^{3}$ & $\mathrm{HC} 2$ (pi) & -9.81 & 190 & 133 \\
\hline 184 & $\mathrm{~N} \mathrm{sp}{ }^{3}$ & HC2(2pi) & 4.73 & 204 & 169 \\
\hline 185 & $\mathrm{~N} \mathrm{sp}^{3}$ & $\mathrm{HCN}$ & -5.80 & 4 & 3 \\
\hline 186 & $\mathrm{~N} \mathrm{sp}^{3}$ & $\mathrm{HCN}(\mathrm{pi})$ & -2.85 & 8 & 6 \\
\hline 187 & $\mathrm{~N} \mathrm{sp}^{3}$ & $\mathrm{HCN}(+)(\mathrm{pi})$ & 16.06 & 4 & 2 \\
\hline 188 & $\mathrm{~N} \mathrm{sp}^{3}$ & $\operatorname{HCN}(2 \mathrm{pi})$ & 0.95 & 12 & 11 \\
\hline 189 & $\mathrm{~N} \mathrm{sp}^{3}$ & $\mathrm{HCO}(\mathrm{pi})$ & 30.19 & 1 & 1 \\
\hline 190 & $\mathrm{~N} \mathrm{sp}^{3}$ & $\mathrm{HCP}$ & -6.83 & 2 & 2 \\
\hline 191 & $\mathrm{~N} \mathrm{sp}{ }^{3}$ & HCS & 17.10 & 2 & 2 \\
\hline 192 & $\mathrm{~N} \mathrm{sp}^{3}$ & HCS(pi) & 9.38 & 22 & 22 \\
\hline 193 & $\mathrm{~N} \mathrm{sp}^{3}$ & $\mathrm{HSi} 2$ & 1.67 & 7 & 2 \\
\hline 194 & $\mathrm{~N} \mathrm{sp}^{3}$ & $\mathrm{C} 3$ & -32.04 & 41 & 37 \\
\hline 195 & $\mathrm{~N} \mathrm{sp}^{3}$ & C3(pi) & -17.08 & 137 & 97 \\
\hline 196 & $\mathrm{~N} \mathrm{sp}^{3}$ & C3(2pi) & -12.64 & 136 & 108 \\
\hline 197 & $\mathrm{~N} \mathrm{sp}^{3}$ & C3(3pi) & 4.26 & 22 & 20 \\
\hline 198 & $\mathrm{~N} \mathrm{sp}^{3}$ & $\mathrm{C} 2 \mathrm{~N}$ & -18.10 & 3 & 3 \\
\hline 199 & $\mathrm{~N} \mathrm{sp}^{3}$ & C2N(pi) & -6.67 & 7 & 5 \\
\hline 200 & $\mathrm{~N} \mathrm{sp}^{3}$ & $\mathrm{C} 2 \mathrm{~N}(+)(\mathrm{pi})$ & 20.95 & 32 & 17 \\
\hline 201 & $\mathrm{~N} \mathrm{sp}{ }^{3}$ & $\mathrm{C} 2 \mathrm{~N}(2 \mathrm{pi})$ & -3.87 & 15 & 14 \\
\hline 202 & $\mathrm{~N} \mathrm{sp}^{3}$ & $\mathrm{C} 2 \mathrm{~N}(3 \mathrm{pi})$ & 1.17 & 6 & 6 \\
\hline 203 & $\mathrm{~N} \mathrm{sp}^{3}$ & $\mathrm{C} 2 \mathrm{~N}(+)(2 \mathrm{pi})$ & -0.16 & 12 & 12 \\
\hline 204 & $\mathrm{~N} \mathrm{sp}^{3}$ & $\mathrm{C} 2 \mathrm{O}$ & -41.10 & 5 & 5 \\
\hline 205 & $\mathrm{~N} \mathrm{sp}^{3}$ & $\mathrm{C} 2 \mathrm{O}(\mathrm{pi})$ & 9.25 & 39 & 15 \\
\hline 206 & $\mathrm{~N} \mathrm{sp}^{3}$ & $\mathrm{C} 2 \mathrm{O}(2 \mathrm{pi})$ & 29.03 & 1 & 1 \\
\hline 207 & $\mathrm{~N} \mathrm{sp}{ }^{3}$ & $\mathrm{C} 2 \mathrm{P}$ & 7.24 & 1 & 1 \\
\hline 208 & $\mathrm{~N} \mathrm{sp}^{3}$ & $\mathrm{C} 2 \mathrm{~S}$ & -25.22 & 3 & 3 \\
\hline 209 & $\mathrm{~N} \mathrm{sp}^{3}$ & C2S(pi) & -22.07 & 1 & 1 \\
\hline 210 & $\mathrm{~N} \mathrm{sp}^{3}$ & C2S(2pi) & -6.25 & 3 & 3 \\
\hline 211 & $\mathrm{~N} \mathrm{sp}^{3}$ & CF2 & -2.10 & 6 & 2 \\
\hline 212 & $\mathrm{~N}(+) \mathrm{sp}^{3}$ & $\mathrm{H} 2 \mathrm{C} 2$ & 4.33 & 19 & 19 \\
\hline 213 & $\mathrm{~N}(+) \mathrm{sp}^{3}$ & $\mathrm{C} 3 \mathrm{O}(-)$ & -33.09 & 1 & 1 \\
\hline 214 & $\mathrm{~N} \mathrm{sp}^{2}$ & $\mathrm{H}=\mathrm{C}$ & 16.94 & 3 & 3 \\
\hline 215 & $\mathrm{~N} \mathrm{sp}^{2}$ & $\mathrm{C}=\mathrm{C}$ & -7.28 & 122 & 101 \\
\hline 216 & $\mathrm{~N} \mathrm{sp}^{2}$ & $\mathrm{C}=\mathrm{N}$ & -11.24 & 64 & 32 \\
\hline 217 & $\mathrm{~N} \mathrm{sp}^{2}$ & $\mathrm{C}=\mathrm{N}(+)$ & 10.95 & 10 & 7 \\
\hline 218 & $\mathrm{~N} \mathrm{sp}^{2}$ & $=\mathrm{CN}$ & -0.51 & 38 & 31 \\
\hline 219 & $\mathrm{~N} \mathrm{sp}^{2}$ & $=\mathrm{CO}$ & 0.98 & 32 & 31 \\
\hline 220 & $\mathrm{~N} \mathrm{sp}^{2}$ & $=\mathrm{CS}$ & -4.17 & 3 & 2 \\
\hline 221 & $\mathrm{~N} \mathrm{sp}^{2}$ & $\mathrm{~N}=\mathrm{N}$ & -0.32 & 10 & 6 \\
\hline 222 & $\mathrm{~N} \mathrm{sp}^{2}$ & $\mathrm{~N}=\mathrm{O}$ & 18.24 & 4 & 2 \\
\hline 223 & $\mathrm{~N}$ aromatic & :C2 & 5.43 & 222 & 128 \\
\hline 224 & $\mathrm{~N}$ aromatic & $: \mathrm{C}: \mathrm{N}$ & -4.60 & 6 & 3 \\
\hline 225 & $\mathrm{~N}(+) \mathrm{sp}^{2}$ & $\mathrm{C}=\mathrm{NO}(-)$ & -19.90 & 4 & 4 \\
\hline 226 & $\mathrm{~N}(+) \mathrm{sp}^{2}$ & $\mathrm{CO}=\mathrm{O}(-)$ & 1.45 & 248 & 163 \\
\hline 227 & $\mathrm{~N}(+) \mathrm{sp}^{2}$ & $=\mathrm{CO} 2(-)$ & -3.88 & 1 & 1 \\
\hline
\end{tabular}


Table 4. Cont.

\begin{tabular}{|c|c|c|c|c|c|}
\hline Entry & Atom Type & Neighbours & Contribution & Occurrences & Molecules \\
\hline 228 & $\mathrm{~N}(+) \mathrm{sp}^{2}$ & $\mathrm{NO}=\mathrm{O}(-)$ & -1.33 & 48 & 31 \\
\hline 229 & $\mathrm{~N}(+) \mathrm{sp}^{2}$ & $\mathrm{O} 2=\mathrm{O}(-)$ & 1.85 & 7 & 5 \\
\hline 230 & $\mathrm{~N}(+) \mathrm{sp}$ & $\mathrm{C \# C}(-)$ & 10.24 & 1 & 1 \\
\hline 231 & $\mathrm{~N}(+) \mathrm{sp}$ & $=\mathrm{N} 2(-)$ & 2.76 & 6 & 3 \\
\hline 232 & $\mathrm{O}$ & $\mathrm{HC}$ & -2.00 & 452 & 254 \\
\hline 233 & $\mathrm{O}$ & $\mathrm{HC}(\mathrm{pi})$ & 3.39 & 478 & 400 \\
\hline 234 & $\mathrm{O}$ & HN & 0.63 & 36 & 12 \\
\hline 235 & $\mathrm{O}$ & $\mathrm{HN}(\mathrm{pi})$ & -1.02 & 19 & 19 \\
\hline 236 & $\mathrm{O}$ & $\mathrm{HP}$ & -8.39 & 2 & 1 \\
\hline 237 & $\mathrm{O}$ & HS & 60.03 & 5 & 2 \\
\hline 238 & $\mathrm{O}$ & $\mathrm{BC}$ & 0.00 & 5 & 5 \\
\hline 239 & $\mathrm{O}$ & $\mathrm{BN}$ & 0.00 & 5 & 5 \\
\hline 240 & $\mathrm{O}$ & $\mathrm{C} 2$ & -4.67 & 357 & 135 \\
\hline 241 & $\mathrm{O}$ & C2(pi) & -5.72 & 740 & 513 \\
\hline 242 & $\mathrm{O}$ & C2(2pi) & -3.04 & 267 & 217 \\
\hline 243 & $\mathrm{O}$ & $\mathrm{CN}$ & -20.33 & 4 & 4 \\
\hline 244 & $\mathrm{O}$ & $\mathrm{CN}(\mathrm{pi})$ & 0.00 & 1 & 1 \\
\hline 245 & $\mathrm{O}$ & $\mathrm{CN}(2 \mathrm{pi})$ & 1.82 & 12 & 11 \\
\hline 246 & $\mathrm{O}$ & $\mathrm{CN}(+)(\mathrm{pi})$ & 0.47 & 7 & 5 \\
\hline 247 & $\mathrm{O}$ & $\mathrm{CO}$ & 1.80 & 8 & 4 \\
\hline 248 & $\mathrm{O}$ & $\mathrm{CP}$ & -6.11 & 47 & 25 \\
\hline 249 & $\mathrm{O}$ & $\mathrm{CP}(\mathrm{pi})$ & 6.35 & 20 & 17 \\
\hline 250 & $\mathrm{O}$ & $\mathrm{CS}(\mathrm{pi})$ & 1.11 & 3 & 3 \\
\hline 251 & $\mathrm{O}$ & CSi & -12.94 & 5 & 2 \\
\hline 252 & $\mathrm{O}$ & N2(2pi) & & & \\
\hline 253 & $\mathrm{O}$ & $\mathrm{N} 2(+)(2 \mathrm{pi})$ & 0.00 & 1 & 1 \\
\hline 254 & $\mathrm{O}$ & $\mathrm{Si} 2$ & 2.53 & 84 & 24 \\
\hline 255 & P3 & $\mathrm{C} 3$ & -6.01 & 3 & 2 \\
\hline 256 & P4 & $\mathrm{C} 3=\mathrm{O}$ & -6.07 & 1 & 1 \\
\hline 257 & P4 & $\mathrm{C}=\mathrm{OF} 2$ & -1.93 & 1 & 1 \\
\hline 258 & P4 & $\mathrm{C}=\mathrm{OFCl}$ & -4.92 & 1 & 1 \\
\hline 259 & P4 & $\mathrm{C}=\mathrm{OCl} 2$ & 6.84 & 1 & 1 \\
\hline 260 & P4 & $\mathrm{N} 2 \mathrm{O}=\mathrm{O}$ & 6.11 & 1 & 1 \\
\hline 261 & P4 & $\mathrm{NO} 2=\mathrm{O}$ & -7.48 & 1 & 1 \\
\hline 262 & P4 & $\mathrm{NOS}=\mathrm{S}$ & 6.11 & 1 & 1 \\
\hline 263 & $\mathrm{P} 4$ & $\mathrm{O} 3=\mathrm{O}$ & -5.29 & 2 & 2 \\
\hline 264 & P4 & $\mathrm{O} 3=\mathrm{S}$ & -3.13 & 13 & 12 \\
\hline 265 & P4 & $\mathrm{CO} 2=\mathrm{O}$ & 0.00 & 1 & 1 \\
\hline 266 & P4 & $\mathrm{CO} 2=\mathrm{S}$ & 7.66 & 2 & 2 \\
\hline 267 & P4 & $\mathrm{O} 2 \mathrm{~S}=\mathrm{S}$ & -5.52 & 7 & 7 \\
\hline 268 & S2 & $\mathrm{HC}$ & -0.29 & 19 & 19 \\
\hline 269 & S2 & $\mathrm{HC}(\mathrm{pi})$ & -11.91 & 2 & 2 \\
\hline 270 & S2 & $\mathrm{C} 2$ & -10.10 & 74 & 47 \\
\hline 271 & S2 & C2(pi) & 1.44 & 44 & 37 \\
\hline 272 & S2 & C2(2pi) & 8.54 & 74 & 60 \\
\hline 273 & S2 & $\mathrm{CN}$ & 0.00 & 3 & 3 \\
\hline 274 & S2 & $\mathrm{CN}(\mathrm{pi})$ & 5.57 & 1 & 1 \\
\hline 275 & S2 & $\mathrm{CS}$ & 1.49 & 8 & 4 \\
\hline 276 & S2 & CS(pi) & 0.18 & 6 & 4 \\
\hline 277 & S2 & $\mathrm{CP}$ & 0.00 & 8 & 8 \\
\hline 278 & S2 & N2(2pi) & -3.71 & 1 & 1 \\
\hline 279 & S4 & $\mathrm{C} 2=\mathrm{O}$ & -10.46 & 6 & 4 \\
\hline 280 & S4 & $\mathrm{C} 2=\mathrm{O} 2$ & -10.18 & 22 & 22 \\
\hline 281 & S4 & $\mathrm{CN}=\mathrm{O} 2$ & 1.23 & 31 & 31 \\
\hline 282 & S4 & $\mathrm{CO}=\mathrm{O} 2$ & 0.00 & 8 & 5 \\
\hline 283 & S4 & $\mathrm{C}=\mathrm{OS}$ & 4.07 & 2 & 2 \\
\hline 284 & S4 & $\mathrm{N} 2=\mathrm{O} 2$ & 4.49 & 2 & 2 \\
\hline
\end{tabular}


Table 4. Cont.

\begin{tabular}{|c|c|c|c|c|c|}
\hline Entry & Atom Type & Neighbours & Contribution & Occurrences & Molecules \\
\hline 285 & $\mathrm{Si}$ & $\mathrm{H} 3 \mathrm{C}$ & 0.00 & 1 & 1 \\
\hline 286 & $\mathrm{Si}$ & $\mathrm{HC} 2 \mathrm{O}$ & -77.65 & 3 & 3 \\
\hline 287 & $\mathrm{Si}$ & $\mathrm{HCO} 2$ & 18.28 & 1 & 1 \\
\hline 288 & $\mathrm{Si}$ & $\mathrm{C} 4$ & -12.05 & 23 & 18 \\
\hline 289 & $\mathrm{Si}$ & $\mathrm{C} 3 \mathrm{O}$ & -15.58 & 14 & 9 \\
\hline 290 & $\mathrm{Si}$ & $\mathrm{C} 3 \mathrm{Cl}$ & -8.02 & 2 & 2 \\
\hline 291 & $\mathrm{Si}$ & $\mathrm{C} 3 \mathrm{Si}$ & -6.42 & 6 & 3 \\
\hline 292 & $\mathrm{Si}$ & $\mathrm{C} 2 \mathrm{~N} 2$ & 0.00 & 7 & 2 \\
\hline 293 & $\mathrm{Si}$ & $\mathrm{C} 2 \mathrm{O} 2$ & 1.03 & 75 & 18 \\
\hline 294 & $\mathrm{Si}$ & $\mathrm{C} 2 \mathrm{Cl} 2$ & -1.79 & 2 & 2 \\
\hline 295 & $\mathrm{Si}$ & $\mathrm{C} 2 \mathrm{Si} 2$ & -10.09 & 34 & 5 \\
\hline 296 & $\mathrm{Si}$ & $\mathrm{CCl} 3$ & 4.64 & 8 & 7 \\
\hline 297 & $\mathrm{Si}$ & $\mathrm{O} 4$ & 13.30 & 1 & 1 \\
\hline 298 & $\mathrm{H}$ & H Acceptor & 6.31 & 153 & 128 \\
\hline 299 & Angle60 & & 0.54 & 120 & 33 \\
\hline 300 & Angle90 & & 2.37 & 138 & 29 \\
\hline 301 & Angle102 & & 0.12 & 1131 & 342 \\
\hline 302 & Endocyclic bonds & No. of single bonds & -4.42 & 5302 & 680 \\
\hline $\mathrm{A}$ & Based on & Valid groups & 188 & & 2809 \\
\hline $\mathrm{B}$ & Goodness of fit & $R^{2}$ & 0.8875 & & 2701 \\
\hline $\mathrm{C}$ & Deviation & Average & 12.33 & & 2701 \\
\hline $\mathrm{D}$ & Deviation & Standard & 16.72 & & 2701 \\
\hline $\mathrm{E}$ & $\mathrm{K}$-fold cv & $\mathrm{K}$ & 10 & & 2637 \\
\hline $\mathrm{F}$ & Goodness of fit & $Q^{2}$ & 0.8727 & & 2637 \\
\hline G & Deviation & Average (cv) & 13.23 & & 2637 \\
\hline $\mathrm{H}$ & Deviation & Standard (cv) & 17.93 & & 2637 \\
\hline
\end{tabular}

The correlation diagram in Figure 9 exhibits a large concentration of the entropy values in the range between 0 and ca. $140 \mathrm{~J} / \mathrm{mol} / \mathrm{K}$; values of $200 \mathrm{~J} / \mathrm{mol} / \mathrm{K}$ or more are exclusively reserved for molecules carrying long, mostly un-branched methylene or poly-ether chains. The histogram (Figure 10) reveals a slight overweight of the positive deviations, indicating a minor trend to predict too low values.

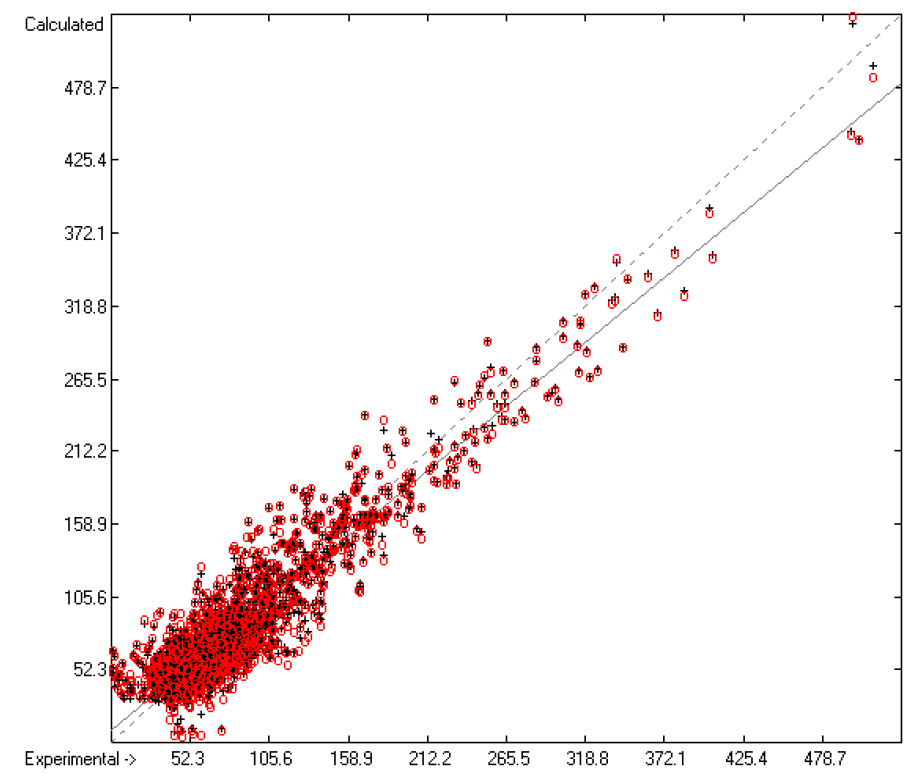

Figure 9. Correlation Diagram of the Entropy-of-Fusion Data $\left(N=2701 ; R^{2}=0.8874 ; Q^{2}=0.8727\right.$; regression line: intercept $=8.6540$; slope $=0.8883$ ). 


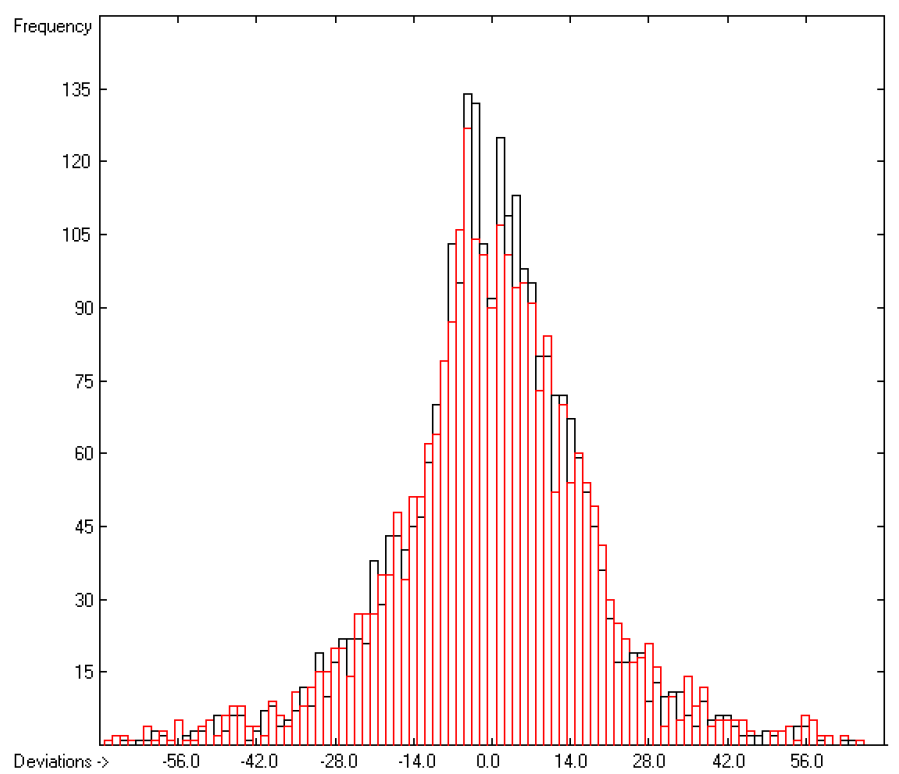

Figure 10. Histogram of the Entropy-of-Fusion Data $(S=17.93 \mathrm{~J} / \mathrm{mol} / \mathrm{K} ;$ Exp. values range: $0.65-513.5 \mathrm{~J} / \mathrm{mol} / \mathrm{K})$.

\subsection{Total Phase-Change Entropy of Liquid Crystals}

Liquid crystals are a class of molecules characterized by the special feature to often exhibit several distinct semiliquid states between their crystalline and isotropic liquid phases, i.e., anisotropic phases which are stable over an extended temperature range. Depending on their intermediate structure these phases are either called meso, cholesteric, smectic or nematic. This strange self-associative behaviour has typically been found with compounds the molecular structure of which contains rigid moieties and highly flexible pendant alkyl or polyether chains of various length, but also with molecules where certain parts exhibit strong intermolecular hydrogen bonds besides moieties of intermolecular inertness. Due to the variability of their entire melting processes resulting from their structural characteristics, the only common entropy term to possibly be generally applicable is the total phase-change entropy, defined as "the sum of all the entropy changes associated with phase transitions occurring from $T=0 \mathrm{~K}$ to the

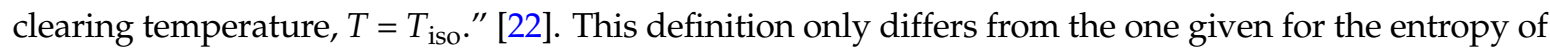
fusion in the previous chapter, in that here not only the potential solid-solid entropy-phase changes but also the entropy changes of the semiliquid intermediate phases are considered. Based on this definition, only the total phase-change entropy data of liquid crystals have been entered into the evaluation of the related atom-group parameters. The only source for these data was the large collection of more than 3000 compounds in the compendium work of Acree, Jr. and Chickos [22]. The parameters calculation finally rested on 2686 compounds, yielding a direct goodness of fit $R^{2}$ of 0.6094 and a cross-validated goodness $Q^{2}$ of 0.5804 with a standard deviation of 32.79, as condensed at the bottom of Table 5 . (In order to compare these data directly with those of the entropy-of-fusion calculation, the special groups "Angle60" and "Angle90" are kept in the parameters list although obviously no compound met any of these two criteria, i.e., bond angle $\leq 90$ or $<60$.) These data compare favourably with those of Acree and Chickos [22], who reported a correlation coefficient of only 0.35 for 627 liquid crystals. The present results, however, required the removal of 56 compounds from the evaluation of the parameters, as their deviation from prediction was much larger than three times the cv-standard deviation. 
Table 5. Atom Groups and their Contributions (in J/mol/K) for Total Phase-Change Entropy Calculations.

\begin{tabular}{|c|c|c|c|c|c|}
\hline Entry & Atom Type & Neighbours & Contribution & Occurrences & Molecules \\
\hline 1 & Const & & 60.14 & 2686 & 2686 \\
\hline 2 & $\mathrm{Csp}{ }^{3}$ & $\mathrm{H} 3 \mathrm{C}$ & 5.33 & 5873 & 2490 \\
\hline 3 & $\mathrm{Csp}$ & $\mathrm{H} 3 \mathrm{~N}$ & 16.05 & 12 & 6 \\
\hline 4 & $\mathrm{Csp} \mathrm{sp}^{3}$ & $\mathrm{H} 3 \mathrm{O}$ & 2.66 & 195 & 172 \\
\hline 5 & $\mathrm{Csp}{ }^{3}$ & $\mathrm{H} 3 \mathrm{Si}$ & 3.08 & 110 & 5 \\
\hline 6 & $\mathrm{Csp}$ & $\mathrm{H} 2 \mathrm{C} 2$ & 4.04 & 30,650 & 2478 \\
\hline 7 & $\mathrm{Csp}$ & $\mathrm{H} 2 \mathrm{CN}$ & -1.70 & 286 & 114 \\
\hline 8 & $\mathrm{Csp}$ & $\mathrm{H} 2 \mathrm{CO}$ & -0.01 & 3584 & 1901 \\
\hline 9 & $\mathrm{Csp}$ & $\mathrm{H} 2 \mathrm{CS}$ & -8.01 & 68 & 42 \\
\hline 10 & $\mathrm{Csp}$ & $\mathrm{H} 2 \mathrm{CCl}$ & -27.41 & 2 & 2 \\
\hline 11 & $\mathrm{Csp}{ }^{3}$ & $\mathrm{H} 2 \mathrm{CBr}$ & -10.24 & 3 & 3 \\
\hline 12 & $\mathrm{Csp}$ & $\mathrm{H} 2 \mathrm{CJ}$ & 30.88 & 1 & 1 \\
\hline 13 & $\mathrm{Csp}$ & $\mathrm{H} 2 \mathrm{CSi}$ & -2.48 & 6 & 3 \\
\hline 14 & $\mathrm{Csp}^{3}$ & $\mathrm{HC} 3$ & -9.84 & 1088 & 414 \\
\hline 15 & $\mathrm{Csp}$ & $\mathrm{HC} 2 \mathrm{~N}$ & -17.47 & 4 & 4 \\
\hline 16 & $\mathrm{Csp}$ & $\mathrm{HC} 2 \mathrm{O}$ & -19.96 & 428 & 324 \\
\hline 17 & $\mathrm{Csp}$ & $\mathrm{HC} 2 \mathrm{~S}$ & -42.59 & 18 & 18 \\
\hline 18 & $\mathrm{Csp}$ & $\mathrm{HC} 2 \mathrm{Cl}$ & -12.96 & 53 & 53 \\
\hline 19 & $\mathrm{Csp}$ & $\mathrm{HC} 2 \mathrm{Br}$ & 6.97 & 4 & 4 \\
\hline 20 & $\mathrm{Csp}$ & $\mathrm{HCO} 2$ & 7.19 & 34 & 28 \\
\hline 21 & $\mathrm{Csp} \mathrm{sp}^{3}$ & HCF2 & -21.83 & 11 & 11 \\
\hline 22 & $\mathrm{Csp}{ }^{3}$ & $\mathrm{C} 4$ & -0.53 & 212 & 120 \\
\hline 23 & $\mathrm{Csp} \mathrm{sp}^{3}$ & $\mathrm{C} 3 \mathrm{O}$ & 12.06 & 10 & 10 \\
\hline 24 & $\mathrm{Csp}$ & $\mathrm{C} 3 \mathrm{~F}$ & -25.29 & 2 & 2 \\
\hline 25 & $\mathrm{Csp}$ & $\mathrm{C} 2 \mathrm{~F} 2$ & 4.67 & 272 & 57 \\
\hline 26 & $\mathrm{Csp}$ & CSF2 & -1.17 & 5 & 5 \\
\hline 27 & $\mathrm{Csp} \mathrm{sp}^{3}$ & $\mathrm{CF} 3$ & -8.30 & 67 & 54 \\
\hline 28 & $C \mathrm{sp}^{3}$ & OF3 & 24.11 & 2 & 2 \\
\hline 29 & $\mathrm{C} \mathrm{sp}^{3}$ & SF3 & -196.06 & 1 & 1 \\
\hline 30 & $\mathrm{Csp} \mathrm{sp}^{2}$ & $\mathrm{H} 2=\mathrm{C}$ & 14.81 & 58 & 56 \\
\hline 31 & $C \mathrm{sp}^{2}$ & $\mathrm{HC}=\mathrm{C}$ & -2.97 & 946 & 440 \\
\hline 32 & $\mathrm{Csp}$ & $\mathrm{HC}=\mathrm{N}$ & -2.07 & 922 & 704 \\
\hline 33 & $\mathrm{Csp} \mathrm{sp}^{2}$ & $\mathrm{HC}=\mathrm{N}(+)$ & 32.39 & 9 & 9 \\
\hline 34 & $\mathrm{Csp}{ }^{2}$ & $\mathrm{HC}=\mathrm{O}$ & 15.32 & 6 & 6 \\
\hline 35 & $\mathrm{Csp}$ & $\mathrm{H}=\mathrm{CN}$ & -16.69 & 43 & 41 \\
\hline 36 & $\mathrm{Csp} \mathrm{sp}^{2}$ & $\mathrm{H}=\mathrm{CO}$ & -2.30 & 28 & 28 \\
\hline 37 & $\mathrm{Csp}^{2}$ & $\mathrm{H}=\mathrm{CS}$ & -4.67 & 2 & 2 \\
\hline 38 & $C \mathrm{sp}^{2}$ & $\mathrm{H}=\mathrm{NS}$ & 74.91 & 1 & 1 \\
\hline 39 & $C \mathrm{sp}^{2}$ & $\mathrm{C} 2=\mathrm{C}$ & -13.21 & 186 & 160 \\
\hline 40 & $\mathrm{Csp} \mathrm{sp}^{2}$ & $\mathrm{C} 2=\mathrm{N}$ & 9.17 & 17 & 17 \\
\hline 41 & $\mathrm{Csp}$ & $\mathrm{C} 2=\mathrm{O}$ & 2.80 & 266 & 202 \\
\hline 42 & $\mathrm{Csp} \mathrm{sp}^{2}$ & $\mathrm{C}=\mathrm{CN}$ & 2.69 & 28 & 21 \\
\hline 43 & $C \mathrm{sp}^{2}$ & $\mathrm{C}=\mathrm{CO}$ & -53.38 & 21 & 21 \\
\hline 44 & $\mathrm{Csp}$ & $\mathrm{C}=\mathrm{CS}$ & -5.66 & 340 & 150 \\
\hline 45 & $\mathrm{Csp}^{2}$ & $\mathrm{C}=\mathrm{CF}$ & 31.70 & 10 & 5 \\
\hline 46 & $\mathrm{Csp} \mathrm{sp}^{2}$ & $\mathrm{CN}=\mathrm{N}$ & -13.68 & 15 & 15 \\
\hline 47 & $\mathrm{Csp} \mathrm{sp}^{2}$ & $\mathrm{CN}=\mathrm{O}$ & -1.75 & 326 & 171 \\
\hline 48 & $\mathrm{C} \mathrm{sp}^{2}$ & $\mathrm{C}=\mathrm{NO}$ & -39.68 & 45 & 30 \\
\hline 49 & $\mathrm{Csp}$ & $\mathrm{CN}=\mathrm{S}$ & -6.95 & 8 & 6 \\
\hline 50 & $\mathrm{C} \mathrm{sp}^{2}$ & $\mathrm{C}=\mathrm{NS}$ & 38.49 & 105 & 77 \\
\hline 51 & $C \mathrm{sp}^{2}$ & $=\mathrm{CNS}$ & -47.14 & 22 & 11 \\
\hline 52 & $\mathrm{Csp}$ & $\mathrm{CO}=\mathrm{O}$ & 8.07 & 3115 & 1580 \\
\hline 53 & $\mathrm{Csp} \mathrm{sp}^{2}$ & $=\mathrm{COS}$ & 128.10 & 5 & 5 \\
\hline 54 & $\mathrm{Csp}$ & $\mathrm{C}=\mathrm{OS}$ & 5.46 & 91 & 81 \\
\hline 55 & $C \mathrm{sp}^{2}$ & $=\mathrm{CSCl}$ & 15.27 & 9 & 9 \\
\hline 56 & $C \mathrm{sp}^{2}$ & $=\mathrm{CSJ}$ & 10.36 & 2 & 2 \\
\hline 57 & $C \mathrm{sp}^{2}$ & $\mathrm{~N}=\mathrm{NS}$ & -11.16 & 72 & 72 \\
\hline 58 & $C \mathrm{sp}^{2}$ & $\mathrm{NO}=\mathrm{O}$ & 38.80 & 6 & 6 \\
\hline 59 & $\mathrm{C} \mathrm{sp}^{2}$ & $=$ NOS & 96.96 & 24 & 12 \\
\hline
\end{tabular}


Table 5. Cont

\begin{tabular}{|c|c|c|c|c|c|}
\hline Entry & Atom Type & Neighbours & Contribution & Occurrences & Molecules \\
\hline 60 & $\mathrm{Csp}{ }^{2}$ & $\mathrm{O} 2=\mathrm{O}$ & 26.06 & 3 & 3 \\
\hline 61 & $\mathrm{C}$ aromatic & $\mathrm{H}: \mathrm{C} 2$ & 3.37 & 28,602 & 2538 \\
\hline 62 & $\mathrm{C}$ aromatic & $\mathrm{H}: \mathrm{C}: \mathrm{N}$ & -0.02 & 151 & 82 \\
\hline 63 & $\mathrm{C}$ aromatic & $\mathrm{H}: \mathrm{C}: \mathrm{N}(+)$ & -9.49 & 12 & 6 \\
\hline 64 & $\mathrm{C}$ aromatic & :C3 & -8.40 & 322 & 107 \\
\hline 65 & $\mathrm{C}$ aromatic & $\mathrm{C}: \mathrm{C} 2$ & -9.58 & 7933 & 2410 \\
\hline 66 & $\mathrm{C}$ aromatic & $\mathrm{C}: \mathrm{C}: \mathrm{N}$ & -38.40 & 89 & 61 \\
\hline 67 & C aromatic & $: \mathrm{C} 2 \mathrm{~N}$ & -13.66 & 1866 & 1124 \\
\hline 68 & C aromatic & $: \mathrm{C} 2 \mathrm{~N}(+)$ & -5.68 & 135 & 119 \\
\hline 69 & $\mathrm{C}$ aromatic & $: \mathrm{C} 2: \mathrm{N}$ & 16.73 & 34 & 33 \\
\hline 70 & $\mathrm{C}$ aromatic & $: \mathrm{C} 2 \mathrm{O}$ & -4.24 & 5711 & 2230 \\
\hline 71 & $\mathrm{C}$ aromatic & :C2S & -29.84 & 116 & 105 \\
\hline 72 & $\mathrm{C}$ aromatic & :C2Si & 10.60 & 4 & 2 \\
\hline 73 & C aromatic & $: \mathrm{C} 2 \mathrm{~F}$ & 4.38 & 525 & 266 \\
\hline 74 & $\mathrm{C}$ aromatic & $: \mathrm{C} 2 \mathrm{Cl}$ & -3.87 & 197 & 151 \\
\hline 75 & $\mathrm{C}$ aromatic & :C2Br & 2.55 & 24 & 23 \\
\hline 76 & $\mathrm{C}$ aromatic & :C2J & -35.42 & 9 & 9 \\
\hline 77 & $\mathrm{C}$ aromatic & $\mathrm{C}: \mathrm{N} 2$ & -43.07 & 27 & 21 \\
\hline 78 & $\mathrm{C}$ aromatic & $: \mathrm{C}: \mathrm{NCl}$ & -51.42 & 2 & 2 \\
\hline 79 & $\mathrm{C}$ aromatic & $\mathrm{N}: \mathrm{N} 2$ & -17.88 & 6 & 3 \\
\hline 80 & $\mathrm{C}$ aromatic & :N2O & -31.16 & 4 & 4 \\
\hline 81 & C sp & $\mathrm{H \# C}$ & 15.40 & 1 & 1 \\
\hline 82 & $\mathrm{Csp}$ & $\mathrm{C \# C}$ & -1.90 & 929 & 304 \\
\hline 83 & C sp & $=\mathrm{C} 2$ & -15.98 & 9 & 9 \\
\hline 84 & C sp & $\mathrm{CHN}$ & 4.72 & 229 & 212 \\
\hline 85 & $\mathrm{Csp}$ & \#CO & 29.96 & 2 & 1 \\
\hline 86 & $\mathrm{Csp}$ & $=\mathrm{N}=\mathrm{O}$ & 0.85 & 3 & 2 \\
\hline 87 & $\mathrm{Csp}$ & $=\mathrm{N}=\mathrm{S}$ & 15.48 & 42 & 42 \\
\hline 88 & C sp & \#NS & 7.49 & 26 & 26 \\
\hline 89 & $\mathrm{~N} \mathrm{sp}^{3}$ & $\mathrm{H} 2 \mathrm{C}$ & -12.08 & 5 & 5 \\
\hline 90 & $\mathrm{~N} \mathrm{sp}^{3}$ & $\mathrm{H} 2 \mathrm{C}(\mathrm{pi})$ & -66.66 & 6 & 6 \\
\hline 91 & $\mathrm{~N} \mathrm{sp}^{3}$ & HC2(pi) & 18.61 & 17 & 9 \\
\hline 92 & $\mathrm{~N} \mathrm{sp}^{3}$ & HC2(2pi) & -4.58 & 233 & 143 \\
\hline 93 & $\mathrm{~N} \mathrm{sp}^{3}$ & $\mathrm{HCN}(\mathrm{pi})$ & -6.87 & 6 & 3 \\
\hline 94 & $\mathrm{~N} \mathrm{sp}^{3}$ & $\mathrm{HCN}(2 \mathrm{pi})$ & 42.99 & 12 & 12 \\
\hline 95 & $\mathrm{~N} \mathrm{sp}^{3}$ & $\operatorname{HCS}(p i)$ & 157.30 & 1 & 1 \\
\hline 96 & $\mathrm{~N} \mathrm{sp}^{3}$ & C3 & -75.12 & 10 & 10 \\
\hline 97 & $\mathrm{~N} \mathrm{sp}^{3}$ & C3(pi) & -20.84 & 64 & 33 \\
\hline 98 & $\mathrm{~N} \mathrm{sp}^{3}$ & C3(2pi) & 8.12 & 34 & 25 \\
\hline 99 & $\mathrm{~N} \mathrm{sp}^{3}$ & C3(3pi) & 29.75 & 24 & 14 \\
\hline 100 & $\mathrm{~N} \mathrm{sp}^{2}$ & $\mathrm{C}=\mathrm{C}$ & 14.07 & 1014 & 778 \\
\hline 101 & $\mathrm{~N} \mathrm{sp}^{2}$ & $\mathrm{C}=\mathrm{N}$ & 9.88 & 722 & 295 \\
\hline 102 & $\mathrm{~N} \mathrm{sp}^{2}$ & $\mathrm{C}=\mathrm{N}(+)$ & 8.87 & 32 & 32 \\
\hline 103 & $\mathrm{~N} \mathrm{sp}^{2}$ & $=\mathrm{CN}$ & -40.91 & 206 & 94 \\
\hline 104 & $\mathrm{~N} \mathrm{sp}^{2}$ & $=\mathrm{CO}$ & 33.53 & 26 & 26 \\
\hline 105 & $\mathrm{~N}$ aromatic & $: \mathrm{C} 2$ & 18.59 & 169 & 125 \\
\hline 106 & $\mathrm{~N}$ aromatic & $: \mathrm{C}: \mathrm{N}$ & 17.07 & 12 & 3 \\
\hline 107 & $\mathrm{~N}(+) \mathrm{sp}^{2}$ & $\mathrm{CO}=\mathrm{O}(-)$ & 0.77 & 94 & 78 \\
\hline 108 & $\mathrm{~N}(+) \mathrm{sp}^{2}$ & $\mathrm{C}=\mathrm{CO}(-)$ & -3.27 & 9 & 9 \\
\hline 109 & $\mathrm{~N}(+) \mathrm{sp}^{2}$ & $\mathrm{C}=\mathrm{NO}(-)$ & 0.00 & 32 & 32 \\
\hline 110 & $\mathrm{~N}(+)$ aromatic & $: \mathrm{C} 2 \mathrm{O}(-)$ & 23.39 & 6 & 6 \\
\hline 111 & $\mathrm{O}$ & $\mathrm{HC}$ & 20.86 & 186 & 70 \\
\hline 112 & $\mathrm{O}$ & $\mathrm{HC}(\mathrm{pi})$ & 16.46 & 202 & 156 \\
\hline 113 & $\mathrm{O}$ & $\mathrm{C} 2$ & 1.72 & 100 & 57 \\
\hline 114 & $\mathrm{O}$ & C2(pi) & -0.12 & 3901 & 2018 \\
\hline 115 & $\mathrm{O}$ & C2(2pi) & -2.52 & 2419 & 1340 \\
\hline 116 & $\mathrm{O}$ & $\mathrm{CN}(2 \mathrm{pi})$ & -4.06 & 26 & 26 \\
\hline 117 & S2 & $\mathrm{HC}(\mathrm{pi})$ & -10.11 & 2 & 2 \\
\hline 118 & S2 & C2 & 12.90 & 18 & 18 \\
\hline
\end{tabular}


Table 5. Cont

\begin{tabular}{cccccc}
\hline Entry & Atom Type & Neighbours & Contribution & Occurrences & Molecules \\
\hline 119 & $\mathrm{~S} 2$ & $\mathrm{C} 2(\mathrm{pi})$ & 14.58 & 55 & 42 \\
120 & $\mathrm{~S} 2$ & $\mathrm{C} 2(2 \mathrm{pi})$ & 15.10 & 379 & 314 \\
121 & $\mathrm{~S} 4$ & $\mathrm{CN}=\mathrm{O} 2$ & -36.49 & 1 & 1 \\
122 & $\mathrm{Si}$ & $\mathrm{C} 3 \mathrm{Si}$ & 0.00 & 10 & 5 \\
123 & $\mathrm{Si}$ & $\mathrm{C} 2 \mathrm{Si} 2$ & -3.55 & 45 & 5 \\
124 & $\mathrm{H}$ & $\mathrm{H}$ Acceptor & -17.84 & 151 & 107 \\
125 & Angle60 & & 0.00 & 0 & 0 \\
126 & Angle90 & & 0.00 & 0 & 0 \\
127 & Angle102 & & 7.37 & 513 & 309 \\
128 & Endocyclic bonds & No of single bonds & -1.14 & 3024 & 2686 \\
$\mathrm{~A}$ & Based on & Valid groups & 108 & & 2663 \\
$\mathrm{~B}$ & Goodness of fit & $R^{2}$ & 0.6094 & & 2663 \\
$\mathrm{C}$ & Deviation & Average & 23.83 & & 2663 \\
$\mathrm{D}$ & Deviation & Standard & 31.62 & & 2643 \\
$\mathrm{E}$ & K-fold cv & K & 10 & & 2643 \\
$\mathrm{~F}$ & Goodness of fit & $Q^{2}$ & 0.5804 & & 2643 \\
$\mathrm{G}$ & Deviation & Average (cv) & 24.65 & & 2643 \\
$\mathrm{H}$ & Deviation & Standard (cv) & 32.79 & &
\end{tabular}

Nevertheless, it was to be expected that the additional entropy terms relating to the semiliquid phases would blur the picture in comparison with the previous chapter, since not only each homologous series of liquid crystals but even individual molecules proceed via different melting pathways. This feature is even observable in the list of outliers where several entire homologous series had to be removed. As a consequence of this inhomogeneity, the scatter of the total phase-change entropy of the liquid crystals in Figure 11 is extraordinarily high, but, as the histogram in Figure 12 shows, is evenly distributed about the regression line. This, and the close similarities of $R^{2}$ and $Q^{2}$ as well as of the direct and the cross-validated standard deviations, collected at the bottom of Table 5, may lead to the assumption that the associated atom-group parameters are reliable enough for phase-change entropy predictions within the class of liquid crystals. Two homologous examples may prove whether this assumption is justified.

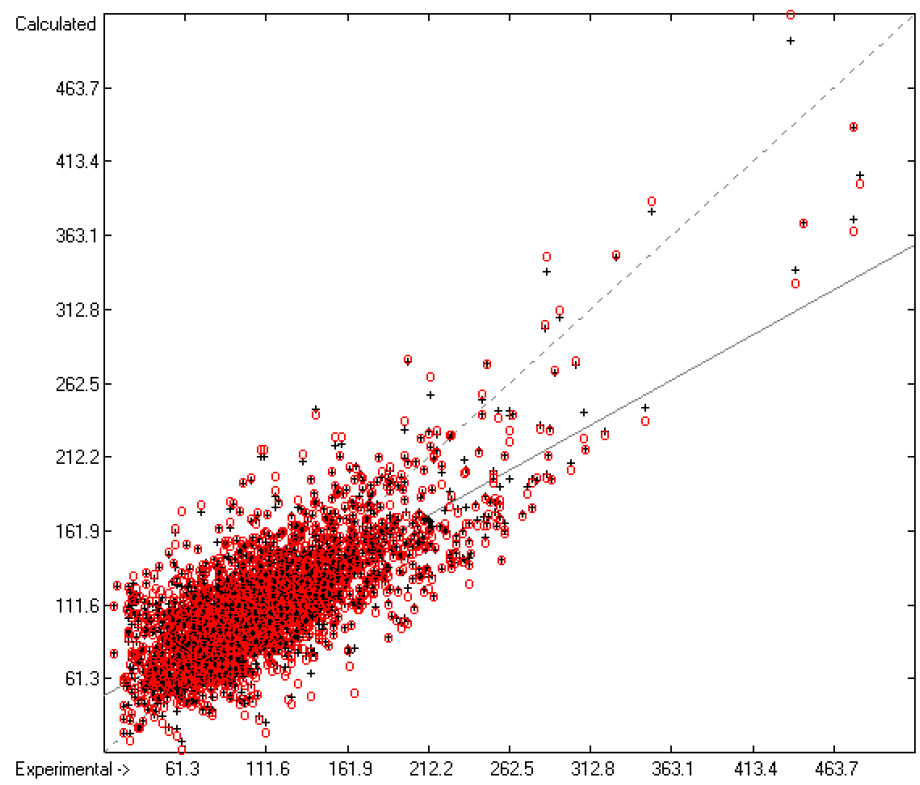

Figure 11. Correlation Diagram of the Total Phase-change Entropy Data $\left(N=2663 ; R^{2}=0.6091\right.$; $Q^{2}=0.5804$; regression line: intercept $=43.5325$, slope $=0.6083$ ). 


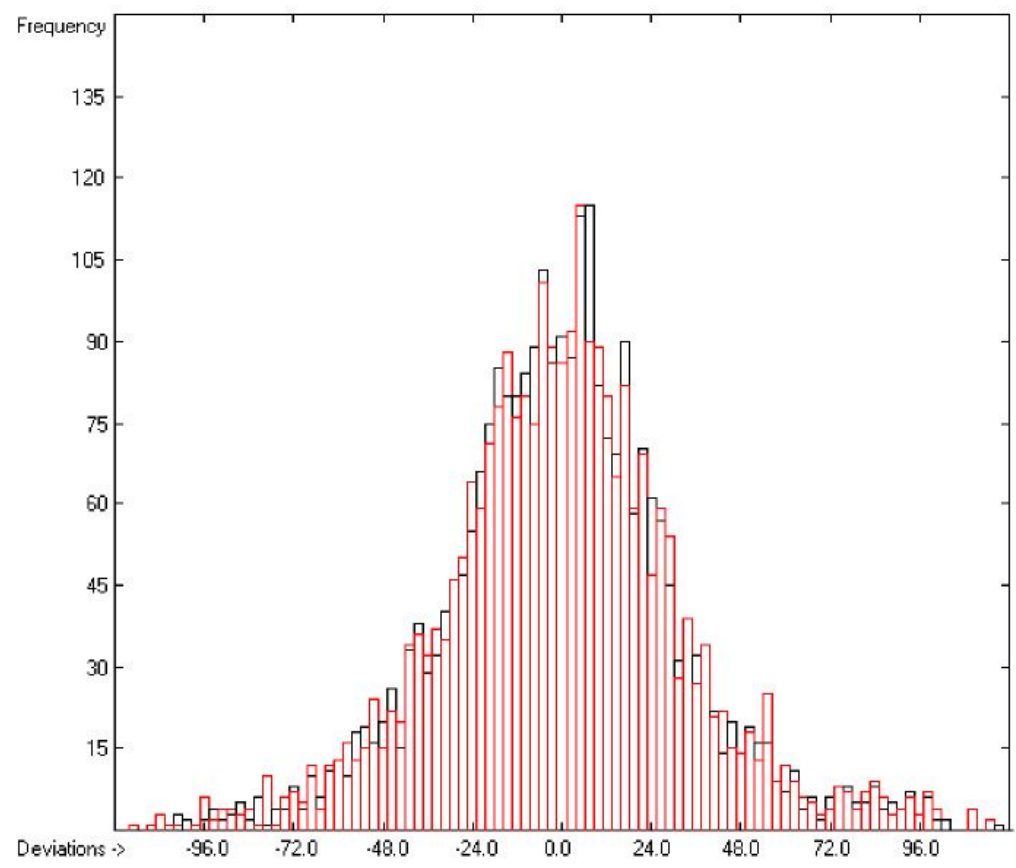

Figure 12. Histogram of the Total Phase-change Entropy Data $(S=32.79 \mathrm{~J} / \mathrm{mol} / \mathrm{K}$; Exp. values range: $17.6-480.76 \mathrm{~J} / \mathrm{mol} / \mathrm{K})$

In Figure 13, the experimental total phase-change entropy data of the liquid-crystal homologues of 7-alkyl-2-(4-cyanophenyl)-fluorene (with alkyl = ethyl, propyl, butyl, pentyl, hexyl, heptyl, octyl and nonyl) are correlated with predicted values, revealing an excellent correlation coefficient $R^{2}$ of 0.9176 . The slope of the regression line, however, is at 0.8830 considerably lower than 1.0. Figure 14 shows the analogous correlation of the homologues of 3-(4-alkyloxyphenylamino)-1-(2-(5-cyanothienyl))-2-propen-1-one (with alkyl = pentyl, hexyl, heptyl, octyl, nonyl, decyl, undecyl, dodecyl, tridecyl and tetradecyl). Here, the correlation coefficient has been calculated to 0.0023 and the slope of the regression line is even slightly negative at -0.0364 . These examples prove that the class of liquid crystals is too heterogeneous for the present atom-group additivity model to be applicable. Consequently, if even within the class of liquid crystals reliable predictions are impossible, attempts to do so outside this class would not make sense at all.

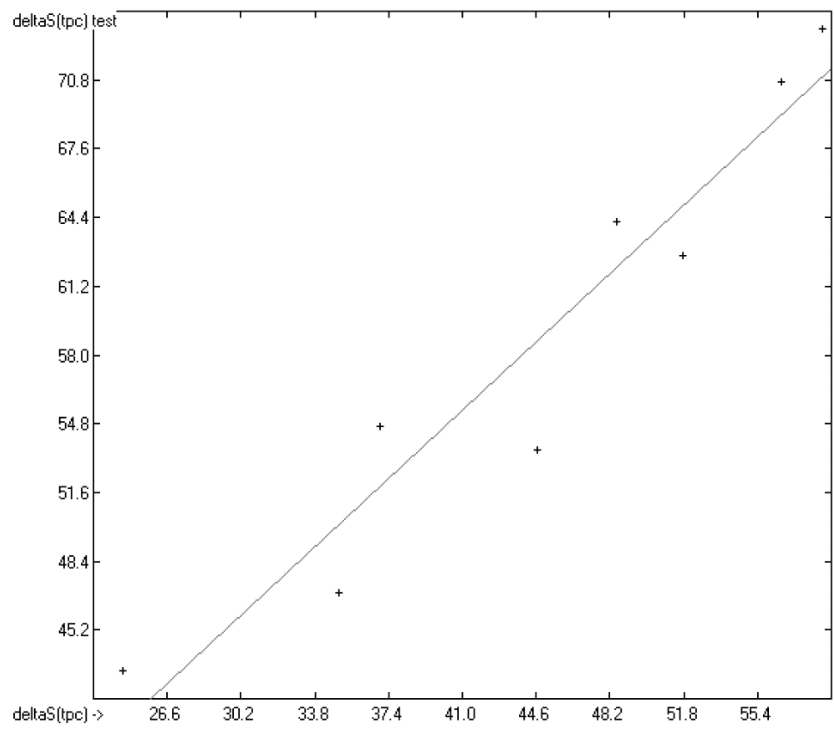

Figure 13. Correlation Diagram of the Total Phase-change Entropy Data of the homologues of 7-alkyl-2-(4-cyanophenyl)-fluorenes. $\left(N=8 ; R^{2}=0.9176 ; \sigma=2.90 \mathrm{~J} / \mathrm{mol} / \mathrm{K}\right)$. 


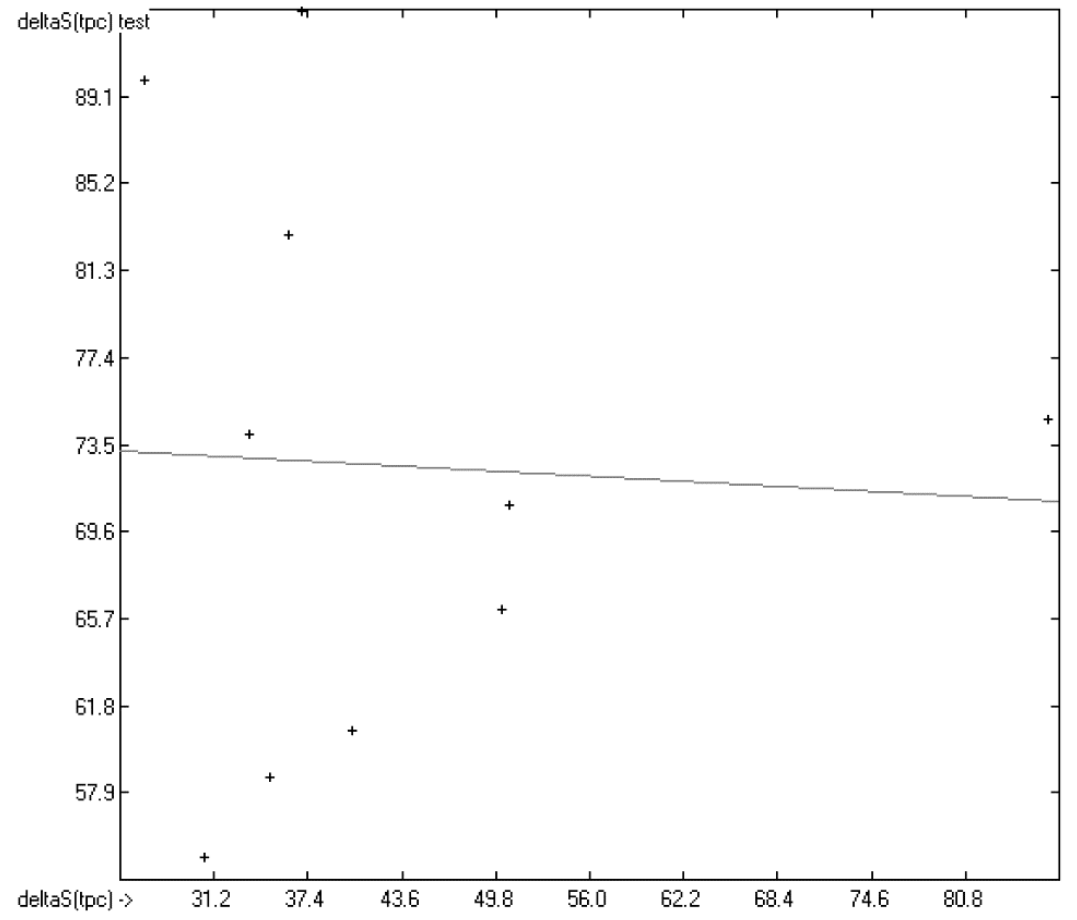

Figure 14. Correlation Diagram of the Total Phase-change Entropy Data of the homologues of 3-(4-alkyloxyphenylamino)-1-(2-(5-cyanothienyl))-2-propen-1-one. $\left(N=10 ; R^{2}=0.0023 ; \sigma=12.29 \mathrm{~J} / \mathrm{mol} / \mathrm{K}\right)$.

\section{Conclusions}

The application of a computer algorithm described in detail in an earlier paper [1], based on the atom-group additivity principle to calculate reliable values of the heat of combustion (and indirectly-formation), $\log \mathrm{P}_{\mathrm{o}} / \mathrm{w}, \log \mathrm{S}$, refractivity, polarizability and toxicity, has successfully been extended to the prediction of the heats of vaporization, sublimation and solvation, and the entropy of fusion of ordinary molecules as well as the total phase-change entropy of liquid crystals. The principle to only accept experimental vaporization and sublimation data measured at or reduced to standard conditions also enabled the indirect calculation of the standard heat of fusion by applying Equation (2). It has been shown, however, that this indirect approach leads to rather rough estimates, yet still comparable to the often large differences of experimental values originating from different authors. The limits of the present method have been reached in the attempt to predict the total phase-change entropy of liquid crystals. In contrast to ordinary molecules which allow the entropy of fusion to be determined in a mutually comparable manner due to a mostly straightforward, uniform melting process, liquid crystals have proven to be an inconsistent class of compounds in that their melting processes pass through several individual semiliquid phases, preventing a standardized approach for the prediction of their phase-change entropy.

On the whole, the present computer algorithm, integrated in a project called ChemBrain IXL, has proven its versatility in that any extension to calculate the presented and future descriptors only requires a few more lines of controlling code to include the corresponding tables and descriptor names. At present, the project covers thermodynamic (heats of combustion, formation, solvation, vaporization, sublimation and fusion as well as entropy of fusion), solubility-related $\left(\log \mathrm{P}_{\mathrm{o}} / \mathrm{w}\right.$ and $\log S$ ), optics-related (refractivity), charge-related (polarizability) and environment-related (toxicity) descriptors. On the other hand, it also shows its limitations where the descriptor is either not addressable by the atom groups (e.g., with $\log B B$ ) or does not describe a uniform characteristic (e.g., the total phase-change entropy). Yet, there is no limit to this ongoing project to extend the number of calculable descriptors beyond the present twelve, provided that there is a number of experimental 
data available that are large enough and reliable. ChemBrain IXL is available from Neuronix Software (www.neuronix.ch, Rudolf Naef, Lupsingen, Switzerland).

Supplementary Materials: The following files are available online.

The entire set of experimental and calculated data of the heat-of-vaporization calculations is available under the name of "S1. Experimental and Calculated Heat-of-Vaporization Data Table.doc"; the corresponding list of compounds is added as an SD file named "S2. Compounds List for Heat-of-Vaporization Calculations.sdf" and the outliers list as an Excel file under the name "S3. Compounds List of Heat-of-Vaporization Outliers.xls".

The list of compounds, their experimental and calculated data and 3D structures of the heat-of-sublimation calculations are available under the names of "S4. Experimental and Calculated Heat-of-Sublimation Data Table.doc" and "S5. Compounds List for Heat-of-Sublimation Calculations.sdf". A list of the outliers has been added under the name of "S6. Compounds List of Heat-of-Sublimation Outliers.xls".

The supplementary material also offers the list of molecules for the enthalpy-of-fusion calculations together with the experimental data under the file names "S7. Experimental and Calculated Heat-of-Fusion Data Table.doc" and "S8. Compounds List for Heat-of-Fusion Calculations.sdf". The list of the outliers is available under the name of "S9. Compounds List of Heat-of-Fusion Outliers.xls".

The heat-of-solvation result list, encompassing the molecule names, experimental and calculated data, are available under the name "S10. Experimental and Calculated Heat-of-Solvation Data Table.doc"; and the molecules list, encompassing their name and 3D coordinates is collected under the name "S11. Compounds List for Heat-of-Solvation Calculations.sdf".

The list of compounds for entropy-of-fusion calculations, together with experimental and calculated data is provided under the name of "S12. Experimental and Calculated Entropy-of-Fusion Data Table.doc". The related compounds' 3D-structures are available in "S13. Compounds List for Entropy-of-Fusion Calculations.sdf", the list of outliers in the Excel sheet called "S14. Compounds List of Entropy-of-Fusion Outliers.xls".

The list of the experimental and calculated data for the total phase-change entropy calculations is provided under "S15. Experimental and Calculated Tpc-Entropy Data Table.doc", the related compounds under the name "S16. Compounds List for Tpc-Entropy Calculations.sdf", and the outliers list under the name "S17. Compounds List of Tpc-Entropy Outliers.xls".

All the figures are available under the names given in the text as gif files, and the tables as doc files.

Acknowledgments: R. Naef is indebted to the library of the University of Basel for allowing him full and free access to the electronic literature database.

Author Contributions: R. Naef developed project ChemBrain and its entire software upon which this paper is based. R. Naef also fed the database, calculated and analysed the results and wrote the paper. W. E. Acree contributed all the experimental data and the majority of the literature references. Beyond this, R. Naef is deeply indebted to W. E. Acree for the many valuable discussions.

Conflicts of Interest: The authors declare no conflict of interest.

\section{References}

1. Naef, R. A Generally Applicable Computer Algorithm Based on the Group Additivity Method for the Calculation of Seven Molecular Descriptors: Heat of Combustion, $\log \mathrm{P}_{\mathrm{O} / \mathrm{W}}, \mathrm{LogS}$, Refractivity, Polarizability, Toxicity and LogBB of Organic Compounds; Scope and Limits of Applicability. Molecules 2015, 20, 18279-18351. [CrossRef] [PubMed]

2. Ghose, A.K.; Crippen, G.M. Atomic physicochemical parameters for three-dimensional structure-directed quantitative structure-activity relationships I. Partition coefficients as a measure of hydrophobicity. J. Comput. Chem. 1986, 7, 565-577. [CrossRef]

3. Ghose, A.K.; Pritchett, A.; Crippen, G.M. Atomic physicochemical parameters for three dimensional structure directed quantitative structure-activity relationships III: Modeling hydrophobic interactions. J. Comput. Chem. 1988, 9, 80-90. [CrossRef]

4. Ghose, A.K.; Crippen, G.M. Atomic Physicochemical parameters for three-dimensional-structure-directed quantitative structure-activity relationships. 2. Modeling dispersive and hydrophobic interactions. J. Chem. Inf. Comput. Sci. 1987, 27, 21-35. [CrossRef] [PubMed]

5. Miller, K.J.; Savchik, J.A. A new empirical Method to calculate Average Molecular Polarizabilities. J. Am. Chem. Soc. 1979, 101, 7206-7213. [CrossRef]

6. Miller, K.J. Additivity methods in molecular polarizability. J. Am. Chem. Soc. 1990, 112, 8533-8542. [CrossRef]

7. Sun, H. A universal molecular descriptor system for prediction of LogP, LogS, LogBB, and absorption. J. Chem. Inf. Comput. Sci. 2004, 44, 748-757. [CrossRef] [PubMed] 
8. Acree, W.E., Jr.; Chickos, J.S. Phase Transition Enthalpy Measurements of Organic and Organometallic Compounds. Sublimation, Vaporization and Fusion Enthalpies from 1880 to 2010. J. Phys. Chem. Ref. Data 2010, 39, 043101. [CrossRef]

9. Roux, M.V.; Temprado, M.; Chickos, J.; Nagano, Y. Critically Evaluated Thermo-chemical Properties of Polycyclic Aromatic Hydrocarbons. J. Phys. Chem. Ref. Data 2008, 37, 1855. [CrossRef]

10. Chickos, J.; Wang, T.; Sharma, E. Hypothetical Thermodynamic Properties: Vapor Pressures and Vaporization Enthalpies of the Even $n$-Alkanes from C40 to C76 at $T=298.15 \mathrm{~K}$ by Correlation-Gas Chromatography. Are the Vaporization Enthalpies a Linear Function of Carbon Number? J. Chem. Eng. Data 2008, 53, 481-491. [CrossRef]

11. Chickos, J.; Lipkind, D. Hypothetical Thermodynamic Properties: Vapor Pressures and Vaporization Enthalpies of the Even $n$-Alkanes from C78 to C92 at $T=298.15 \mathrm{~K}$ by Correlation-Gas Chromatography. J. Chem. Eng. Data 2008, 53, 2432-2440. [CrossRef]

12. Chickos, J.; Hanshaw, W. Vapor Pressures and Vaporization Enthalpies of the $n$-Alkanes from C21 to C30 at $T=298.15 \mathrm{~K}$ by Correlation Gas Chromatography. J. Chem. Eng. Data 2004, 49, 77-85. [CrossRef]

13. Chickos, J.; Hanshaw, W. Vapor Pressures and Vaporization Enthalpies of the n-Alkanes from C31 to C38 at $T=298.15 \mathrm{~K}$ by Correlation Gas Chromatography. J. Chem. Eng. Data 2004, 49, 620-630. [CrossRef]

14. Wilson, J.; Gobble, C.; Chickos, J. Vaporization, Sublimation, and Fusion Enthalpies of Some Saturated and Unsaturated Long Chain Fatty Acids by Correlation Gas Chromatography. J. Chem. Eng. Data 2015, 60, 202-212. [CrossRef]

15. Abraham, M.H. Scales of Hydrogen-bonding: Their Construction and Application to Physicochemical and Biochemical Processes. Chem. Rev. 1993, 22, 73-83. [CrossRef]

16. Abraham, M.H.; Chadha, H.S.; Whinting, G.S.; Mitchell, R.C. Hydrogen-bonding. 32. An Analysis of Water-Octanol and Water-Alkane Partitioning and the $\Delta \log P$ Parameter of Seiler. J. Pharm. Sci. 1994, 83, 1085-1100. [CrossRef] [PubMed]

17. Abraham, M.H.; Zissimos, A.M.; Acree, W.E. Partition of solutes from the gas phase and from water to wet and dry di- $n$-butyl Ether: A linear free energy relationship analysis. Phys. Chem. Chem. Phys. 2001, 3, 3732-3736. [CrossRef]

18. Abraham, M.H.; Le, J. The Correlation and Prediction of the Solubility of Compounds in Water using an amended Solvation Energy Relationship. J. Pharm. Sci. 1999, 88, 868-880. [CrossRef] [PubMed]

19. Jover, J.; Bosque, R.; Sales, J. Determination of Abraham Solute Parameters from Molecular Structure. J. Chem. Inf. Comput. Sci. 2004, 44, 1098-1106. [CrossRef] [PubMed]

20. Cabani, S.; Gianni, P.; Mollica, V.; Lepori, L. Group contributions to the thermodynamic properties of non-ionic organic solutes in dilute aqueous solution. J. Sol. Chem. 1981, 10, 563-595. [CrossRef]

21. Chickos, J.S.; Acree, W.E., Jr.; Liebman, J.F. Estimating Solid-Liquid Phase Change Enthalpies and Entropies. J. Phys. Chem. Ref. Data 1999, 28, 1535-1673. [CrossRef]

22. Acree, W.E., Jr.; Chickos, J.S. Phase Change Enthalpies and Entropies of Liquid Crystals. J. Phys. Chem. Ref. Data 2006, 35, 1051-1330. [CrossRef]

23. Almeida, A.R.R.; Monte, M.J.S. Vapour pressures and phase transition properties of four substituted acetophenones. J. Chem. Thermodyn. 2016, 107, 42-50. [CrossRef]

24. Gobble, C.; Vikman, J.; Chickos, J.S. Evaluation of the Vaporization Enthalpies and Liquid Vapor Pressures of $(R)$-Deprenyl, (S)-Benzphetamine, Alverine, and a Series of Aliphatic Tertiary Amines by Correlation Gas Chromatography at $T / K=298.15$. J. Chem. Eng. Data 2014, 59, 2551-2562. [CrossRef]

25. Miroshnichenko, E.A.; Kon'kova, T.S.; Pashchenko, L.L.; Matyushin, Y.N.; Inozemtsev, Y.O.; Tartakovskii, V.A. Energy characteristics of nitrooxazolidines and their radicals. Russ. Chem. Bull. Int. Ed. 2016, 65, 1876-1878. [CrossRef]

26. Emel'yanenko, V.N.; Zaitseva, K.V.; Nagrimanov, R.N.; Solomonov, B.N.; Verevkin, S.P. Benchmark Thermondynamic Properties of Methyl- and Methoxy-Benzamides: Comprehensive Experimental and Theoretical Study. J. Phys. Chem. A 2016, 120, 8419-8429. [CrossRef] [PubMed]

27. Gobble, C.; Gutterman, A.; Chickos, J.S. Some thermodynamic properties of benzocaine. Struct. Chem. 2013, 24, 1903-1907. [CrossRef]

28. Keating, L.; Harris, H.H.; Chickos, J.S. Vapor pressures and vaporization enthalpy of (-) $\alpha$-bisabolol and (dl) menthol by correlation gas chromatography. J. Chem. Thermodyn. 2017, 107, 18-25. [CrossRef] 
29. Sanchez-Buläs, T.; Cruz-Väsquez, O.; Hernändez-Obregon, J.; Rojas, A. Enthalpies of fusion, vaporisation and sublimation of crown ethers determined by thermogravimetry and differential scanning calorimetry. Thermochim. Acta 2017, 650, 123-133. [CrossRef]

30. Panneerselvam, K.; Anthony, M.P.; Srinivasan, T.G.; Rao, P.R.V. Enthalpies of vaporization of N,N-dialkyl monamides at 298.15K. Thermochim. Acta 2009, 495, 1-4. [CrossRef]

31. Gobble, C.; Walker, B.; Chickos, J.S. The Vaporization Enthalpy and Vapor Pressure of Fenpropidin and Phencyclidine (PCP) at $T / K=298.15$ by Correlation Gas Chromatography. J. Chem. Eng. Data 2016, 61, 896-902. [CrossRef]

32. Kozlovskiy, M.; Gobble, C.; Chickos, J.S. Vapor pressures and vaporization enthalpies of a series of esters used in flavors by correlation gas chromatography. J. Chem. Thermodyn. 2015, 86, 65-74. [CrossRef]

33. Costa, J.C.S.; Lima, C.F.R.A.C.; Mendes, A.; Santos, L.M.N.B.F. Fluorination effect on the thermodynamic properties of long-chain hydrocarbons and alcohols. J. Chem. Thermodyn. 2016, 102, 378-385. [CrossRef]

34. Simmons, D.; Chickos, J. Enthalpy of vaporization and vapor pressure of whiskey lactone and menthalactone by correlation gas chromatography. J. Chem. Thermodyn. 2017, 110, 65-70. [CrossRef]

35. Oliveira, J.A.S.A.; Oliveira, T.S.M.; Gaspar, A.; Borges, F.; Ribeiro da Silva, M.D.M.C.; Monte, M.J.S. Study on the volatility of halogenated fluorenes. Chemosphere 2016, 157, 25-32. [CrossRef] [PubMed]

36. Maxwell, R.; Chickos, J. An Examination of the Thermodynamics of Fusion, Vaporization, and Sublimation of Ibuprofen and Naproxen by Correlation Gas Chromatography. J. Pharm. Sci. 2012, 101, 805-814. [CrossRef] [PubMed]

37. Mori, M.; Rath, N.; Gobble, C.; Chickos, J. Vaporization, Sublimation Enthalpy, and Crystal Structures of Imidazo[1,2-a]pyrazine and Phthalazine. J. Chem. Eng. Data 2016, 61, 370-379. [CrossRef]

38. Goodrich, S.; Hasanovic, J.; Gobble, C.; Chickos, J.S. Vaporization Enthalpies and Vapor Pressures of Some Insect Pheromones by Correlation Gas Chromatography. J. Chem. Eng. Data 2016, 61, 1524-1530. [CrossRef]

39. Freitas, V.L.S.; Silva, C.A.O.; Paiva, M.A.T.; Ribeiro da Silva, M.D.M.C. Energetic effects of alkyl groups (methyl and ethyl) on the nitrogen of the morpholine structure. J. Therm. Anal. Calorim. 2017, 121, 1059-1071. [CrossRef]

40. Althoff, M.A.; Grieger, K.; Härtel, M.A.C.; Karaghiosoff, K.L.; Klapötke, T.M.; Metzulat, M. Application of the Transpiration Method to Determine the Vapor Pressure and Related Physico-Chemical Data of Low Volatile, Thermolabile, and Toxic Organo(thio)phosphates. J. Phys. Chem. A 2017, 121, 2603-2609. [CrossRef] [PubMed]

41. Gobble, C.; Chickos, J.; Verevkin, S.P. Vapor Pressures and Vaporization Enthalpies of a Series of Dialkyl Phthalates by Correlation Gas Chromatography. J. Chem. Eng. Data 2014, 59, 1353-1365. [CrossRef]

42. Brunetti, B.; Lapi, A.; Ciprioti, S.V. Thermodynamic study on six tricyclic nitrogen heterocyclic compounds by thermal analysis and effusion techniques. Thermochim. Acta 2016, 636, 71-84. [CrossRef]

43. Emel'yanenko, V.N.; Kaliner, M.; Strassner, T.; Verevkin, S.P. Thermochemical properties of different 1-(R-phenyl)-1H-imidazoles. Fluid Phase Equilib. 2017, 433, 40-49. [CrossRef]

44. Antón, V.; Artigas, H.; Muñoz-Embid, J.; Artal, M.; Lafuente, C. Thermophysical study of 2-acetylthiophene: Experimental and modelled results. Fluid Phase Equilib. 2017, 433, 126-134. [CrossRef]

45. Portnova, S.V.; Krasnykh, E.L.; Levanova, S.V. Temperature Dependences of Saturated Vapor Pressure and the Enthalpy of Vaporization of n-Pentyl Esters of Dicarboxylic Acids. Russ. J. Phys. Chem. A 2016, 90, 990-993. [CrossRef]

46. Lepori, L.; Matteoli, E.; Gianni, P. Vapor Pressure and Its Temperature Dependence of 28 Organic Compounds: Cyclic Amines, Cyclic Ethers, and Cyclic and Open Chain Secondary Alcohols. J. Chem. Eng. Data 2017, 62, 194-203. [CrossRef]

47. Lima, C.F.R.A.C.; Rodrigues, A.S.M.C.; Santos, L.M.N.B.F. Effect of Confined Hindrance in Polyphenylbenzenes. J. Phys. Chem. A 2017, 121, 2475-2481. [CrossRef] [PubMed]

48. Abboud, J.-L.M.; Alkorta, I.; Davalos, J.Z.; Koppel, I.A.; Koppel, I.; Lenoir, D.; Martínez, S.; Mishima, M. The Thermodynamic Stability of Adamantylideneadamantane and Its Proton- and Electron-Exchanges. Comparison with Simple Alkenes. Bull. Chem. Soc. Jpn. 2016, 89, 762-769. [CrossRef]

49. Silva, A.L.R.; Ribeira da Silva, M.D.M.C. Comprehensive Thermochemical Study of Cyclic Five- and Six-Membered $N, N^{\prime}$-Thioureas. J. Chem. Eng. Data 2017. [CrossRef] 
50. Carvalho, T.M.T.; Amaral, L.M.P.F.; Morais, V.M.F.; Ribeiro da Silva, M.D.M.C. Energetic Effect of the Carboxylic Acid Functional Group in Indole Derivatives. J. Phys. Chem. A 2017, 121, 2980-2989. [CrossRef] [PubMed]

51. Freitas, V.L.S.; Lima, A.C.M.O.; Sapei, E.; Ribeiro da Silva, M.D.M.C. Comprehensive thermophysical and thermochemical studies of vanillyl alcohol. J. Chem. Thermodyn. 2016, 102, 287-292. [CrossRef]

52. Lopes, C.S.D.; Agapito, F.; Bernardes, C.E.S.; Minas da Piedade, M.E. Thermochemistry of 4-HOC ${ }_{6} \mathrm{H}_{4} \mathrm{COR}$ $\left(\mathrm{R}=\mathrm{H}, \mathrm{CH}_{3}, \mathrm{C}_{2} \mathrm{H}_{5}, n-\mathrm{C}_{3} \mathrm{H}_{7}, n-\mathrm{C}_{4} \mathrm{H}_{9}, n-\mathrm{C}_{5} \mathrm{H}_{11}\right.$, and $\left.n-\mathrm{C}_{6} \mathrm{H}_{13}\right)$ compounds. J. Chem. Thermodyn. 2017, 104, 281-287. [CrossRef]

53. Emel'yanenko, V.N.; Nagrimanov, R.N.; Solomonov, B.N.; Verevkin, S.P. Adamantanes: Benchmarking of thermochemical properties. J. Chem. Thermodyn. 2016, 101, 130-138. [CrossRef]

54. Nagrimanov, R.N.; Solomonov, B.N.; Emel'yanenko, V.N.; Verevkin, S.P. Six-membered ring aliphatic compounds: A search for regularities in phase transitions. Thermochim. Acta 2016, 638, 80-88. [CrossRef]

55. Blokhina, S.; Sharapova, A.; Ol'khovich, M.; Perlovich, G. Sublimation thermodynamics of four fluoroquinolone antimicrobial compounds. J. Chem. Thermodyn. 2017, 105, 37-43. [CrossRef]

56. Flores, H.; Ledo, J.M.; Hernandez-Pérez, J.M.; Camarillo, E.A.; Sandoval-Lira, J.; Amador, M.P. Thermochemical and theoretical study of 2-oxazolidinone and 3-acetyl-2-oxazolidinone. J. Chem. Thermodyn. 2016, 102, 386-391. [CrossRef]

57. Emel'yanenko, V.N.; Nagrimanov, R.N.; Verevkin, S.P. Benchmarking thermochemical experiments and calculations of nitrogen-containing substituted adamantanes. J. Thermal. Anal. Calorim. 2017, 128, 1535. [CrossRef]

58. Oliveira, J.A.S.A.; Freitas, V.L.S.; Notario, R.; da Silva, M.D.M.C.R.; Monte, M.J.S. Thermodynamic properties of 2,7-di-tert-butylfluorene-An experimental and computational study. J. Chem. Thermodyn. 2016, 101, 115-122. [CrossRef]

59. Carvalho, T.M.T.; Amaral, L.M.P.F.; Morais, V.M.F.; Ribeiro da Silva, M.D.M.C. Calorimetric and computational studies for three nitroimidazole isomers. J. Chem. Thermodyn. 2016, 105, 267-275. [CrossRef]

60. Chickos, J.S.; Acree, W.E., Jr. Total phase change entropies and enthalpies. An update on fusion enthalpies and their estimation. Thermochim. Acta 2009, 495, 5-13. [CrossRef]

61. Wang, L.; Xing, C.; Zhao, L.; Xu, L.; Liu, G. Measurement and correlation of solubility of 2-chloro-3-(trifluoromethyl)pyridine in pure solvents and ethanol $+n$-propanol mixtures. J. Mol. Liq. 2017, 238, 470-477. [CrossRef]

62. Guenthner, A.J.; Ramirez, S.M.; Ford, M.D.; Soto, D.; Boatz, J.A.; Ghiassi, K.B.; Mabry, J.M. Organic Crystal Engineering of Thermosetting Cyanate Ester Monomers: Influence of Structure on Melting Point. Cryst. Growth Des. 2016, 16, 4082-4093. [CrossRef]

63. Trache, D.; Khimeche, K.; Dahmani, A. Study of (Solid-Liquid) Phase Equilibria for Mixtures of Energetic Material Stabilizers and Prediction for Their Subsequent Performance. Int. J. Thermophys. 2013, 34, 226-239. [CrossRef]

64. Eckert, K.-A.; Dasgupta, S.; Selge, B.; Ay, P. Solid liquid phase diagrams of binary fatty acid mixtures_Palmitic/stearic with oleic/linoleic/linolenic acid mixture. Thermochim. Acta 2016, 630, 50-63. [CrossRef]

65. Blokhina, S.; Sharapova, A.; Ol'khovich, M.; Volkova, T.; Perlovich, G. Studying the sublimation thermodynamics of ethionamide and pyridinecarbothioamide isomers by transpiration method. Thermochim. Acta 2015, 622, 97-102. [CrossRef]

66. Forte, A.; Melo, C.I.; Bogel-Lukasik, R.; Bogel-Lukasik, E. A favourable solubility of isoniazid, an antitubercular antibiotic drug, in alternative solvents. Fluid Phase Equil. 2012, 318, 89-95. [CrossRef]

67. Carletta, A.; Meinguet, C.; Wouters, J.; Tilborg, A. Solid-State Investigation of Polymorphism and Tautomerism of Phenylthiazole-thione: A Combined Crystallographic, Calorimetric, and Theoretical Survey. Cryst. Growth Des. 2015, 15, 2461-2473. [CrossRef]

68. Leitner, J.; Jurik, S. DSC study and thermodynamic modelling of the system paracetamol-o-acetylsalicylic acid. J. Therm. Anal. Calorim. 2017. [CrossRef]

69. Mintz, C.; Clark, M.; Acree, W.E., Jr.; Abraham, M.H. Enthalpy of Solvation Correlations for Gaseous Solutes Dissolved in Water and in 1-Octanol Based on the Abraham Model. J. Chem. Inf. Model. 2007, 47, 115-121. [CrossRef] [PubMed] 
70. Catalan, J.; Couto, A.; Gomez, J.; Saiz, J.L.; Laynez, J. Towards a solvent acidity scale: The calorimetry of the N-methyl imidazole probe. J. Chem. Soc. Perkin Trans. 2 1992, 7, 1181-1185. [CrossRef]

71. Spencer, J.N.; Hovick, J.W. Solvation of urea and methyl-substituted ureas by water and DMF. Can. J. Chem. 1988, 66, 562-565. [CrossRef]

72. Gatta, G.D.; Badea, E. Thermodynamics of Solvation of Urea and Some Monosubstituted N-Alkylureas in Water at 298.15 K. J. Chem. Eng. Data 2007, 52, 419-425. [CrossRef]

73. Rouw, A.; Somsen, G. Solvation and Hydrophobic Hydration of Alkyl-substituted Ureas and Amides in N,N-Dimethylformamide + Water Mixtures. J. Chem. Soc. Faraday Trans. 1 1982, 78, 3397-3408. [CrossRef]

74. Badea, E.; della Gatta, G.; Jozwiak, M.; Giancola, C. Hydration of Thiourea and Mono-, Di-, and Tetra-N-Alkylthioureas at Infinite Dilution: A Thermodynamic Study at a Temperature of 298.15 K. J. Chem. Eng. Data 2011, 56, 4778-4785. [CrossRef]

75. Stimson, E.R.; Schrier, E.E. Calorimetric Investigation of Salt-Amide Interactions in Aqueous Solution. J. Chem. Eng. Data 1974, 19, 354-358. [CrossRef]

76. Batov, D.V.; Zaichikov, A.M. Group Contributions to the Enthalpy Characteristics of Solutions of Formic and Acetic Acid Amides in Water-1,2-Propanediol Mixtures. Russ. J. Gen. Chem. 2003, 73, 511-518. [CrossRef]

77. Starzewski, P.; Wadsö, I.; Zielenkiewicz, W. Enthalpies of vaporization of some N-alkylamides at $298.15 \mathrm{~K}$. J. Chem. Thermodyn. 1984, 16, 331-334. [CrossRef]

78. Morgan, K.M.; Kopp, D.A. Solvent effects on the stability of simple secondary amides. J. Chem. Soc. Perkin Trans. 2 1998, 2759-2763. [CrossRef]

79. Teplitsky, A.B.; Glukhova, O.T.; Sukhodub, L.F.; Yanson, I.K.; Zielenkiewicz, A.; Zielenkiewicz, W.; Kosinski, J.; Wierzchowski, K.L. Thermochemistry of aqueous Solutions of alkylated Nucleic Acid Bases. IV. Enthalpies of 5-Alkyluracils. Biophys. Chem. 1982, 15, 139-147. [CrossRef]

80. Zielenkiewicz, W.; Szterner, P.; Kaminski, M. Vapor Pressures, Molar Enthalpies of Sublimation, and Molar Enthalpies of Solution in Water of Selected Amino Derivatives of Uracil and 5-Nitrouracil. J. Chem. Eng. Data 2003, 48, 1132-1136. [CrossRef]

81. Zielenkiewicz, W.; Szterner, P. Vapor Pressures, Molar Enthalpies of Sublimation, and Molar Enthalpies of Solution in Water of 5-(Trifluoromethyl)uracil. J. Chem. Eng. Data 2004, 49, 1197-1200. [CrossRef]

82. Zielenkiewicz, W.; Szterner, P. Thermodynamic Investigation of Uracil and Its Halo Derivatives. Enthalpies of Solution and Solvation in Methanol. J. Chem. Eng. Data 2005, 50, 1139-1143. [CrossRef]

83. Zhou, Y.; Wang, J.; Fang, B.; Guo, N.; Xiao, Y.; Hao, H.; Bao, Y.; Huang, X. Solubility and dissolution thermodynamic properties of 2-Cyano-4'-methylbiphenyl in binary solvent mixtures. J. Mol. Liq. 2017, 236, 298-307. [CrossRef]

84. Zhang, Q.-A.; Du, C.-J. Solubility of cyclohexyl-phosphoramidic acid diphenyl ester in selected solvents. J. Mol. Liq. 2015, 211, 527-533. [CrossRef]

85. Zhao, F.-Q.; Pei, C.; Hu, R.-Z.; Yang, L.; Zhang, Z.-Z.; Zhou, Y.-S.; Yang, X.-W.; Yin, G.; Gao, S.L.; Shi, Q.-Z. Thermochemical properties and non-isothermal decomposition reaction kinetics of 3,4-dinitrofurazanfuroxan (DNTF). J. Hazard. Mat. 2004, A113, 67-71.

Sample Availability: Sample Availability: Not available.

(C) 2017 by the authors. Licensee MDPI, Basel, Switzerland. This article is an open access article distributed under the terms and conditions of the Creative Commons Attribution (CC BY) license (http:/ / creativecommons.org/licenses/by/4.0/). 